\title{
Animal innovation defined and operationalized
}

\author{
Grant Ramsey \\ Department of Philosophy, University of Notre Dame, Notre Dame, IN \\ 46556-4619 \\ grant.ramsey@nd.edu http://philosophy.nd.edu/people/all/ \\ profiles/ramsey-grant/index.shtml \\ Meredith L. Bastian \\ Department of Biological Anthropology and Anatomy, Duke University, \\ Durham, NC 27708-0383 \\ mlb22@duke.edu http://fds.duke.edu/db/aas/BAA $/$ grad $/ \mathrm{mlb22}$ \\ Carel van Schaik \\ Anthropological Institute and Museum, University of Zürich, 8057 Zürich, \\ Switzerland \\ vschaik@aim.unizh.ch \\ http://www.aim.unizh.ch/Members/vanschaik.html
}

\begin{abstract}
Innovation is a key component of most definitions of culture and intelligence. Additionally, innovations may affect a species' ecology and evolution. Nonetheless, conceptual and empirical work on innovation has only recently begun. In particular, largely because the existing operational definition (first occurrence in a population) requires long-term studies of populations, there has been no systematic study of innovation in wild animals. To facilitate such study, we have produced a new definition of innovation: Innovation is the process that generates in an individual a novel learned behavior that is not simply a consequence of social learning or environmental induction. Using this definition, we propose a new operational approach for distinguishing innovations in the field. The operational criteria employ information from the following sources: (1) the behavior's geographic and local prevalence and individual frequency; (2) properties of the behavior, such as the social role of the behavior, the context in which the behavior is exhibited, and its similarity to other behaviors; (3) changes in the occurrence of the behavior over time; and (4) knowledge of spontaneous or experimentally induced behavior in captivity. These criteria do not require long-term studies at a single site, but information from multiple populations of a species will generally be needed. These criteria are systematized into a dichotomous key that can be used to assess whether a behavior observed in the field is likely to be an innovation.
\end{abstract}

Keywords: behavior, culture, improvisation, innovation, invention, operational definition, social learning

\section{Introduction}

The study of animal innovation is vitally important to the study of animal culture, evolution, ecology, and intelligence. Innovation is a key component of most definitions of culture (Imanishi 1952; Kummer 1971; overviews in McGrew 1998; Rendell \& Whitehead 2001) and intelligence (Reader \& Laland 2002; van Schaik \& Pradhan 2003). Because innovations can affect fitness, they can play an important role in a species' ecology and evolution (Giraldeau et al. 1994; Lefebvre et al. 2004). In spite of this, innovation itself has been infrequently studied, both conceptually and empirically (Kummer \& Goodall 1985).

Reader and Laland's (2003b) edited volume recently opened debate on the topic of animal innovation, offering several important theoretical contributions and reviewing the results of previous attempts to study innovation (see also Huffman \& Hirata 2003). They also developed an operational definition of innovation that involves observing the first occurrence of a novel learned behavior in a population: "Innovation is a process that results in new or modified learned behaviour and that introduces novel behavioural variants into a population's repertoire." (Reader \& Laland 2003a, p. 14). Comparative studies (e.g., Lefebvre et al. 1997; Reader \& Laland 2002) have implicitly relied on similar definitions (although innovations did not always come from populations subject to long-term monitoring). At present, most existing observations of innovation refer to responses to human-made changes in the environment (many examples of the innovations are compiled in comparative studies, e.g., Lefebvre et al. 1997) or to novel behaviors in captivity (e.g., Reader 2003; Reader \& Laland 2001). The currently used operationalization makes it possible to examine the features of individuals or the ecological or social settings that affect the likelihood of innovation, the characteristics of innovators, such as their sex, age, or social position, the psychological processes involved (Reader 2003; Reader \& Laland 2001), as well as the spread of these novelties (Boesch 1995; Hauser 1988). Unfortunately, sample sizes in the wild will often be too small to study features of the innovative behaviors themselves.

Despite being the best attempt to date at defining innovation, Reader and Laland's (2003a) definition has its limitations. Under their definition, the rates of 
innovation production (innovativeness) and also the size of the innovation repertoire are necessarily functions of the size of the population(s) studied, as well as how extensively the species has been studied, thereby precluding unbiased estimates of innovation repertoires. Most long-term field studies will only witness the origin of a few innovations at best, and thus possibly record only a small and perhaps biased subset of the innovations present in the population. Because their definition classifies as innovations only the subset of behaviors that arose during the observation period, we cannot systematically compare innovation repertoires between populations or species, nor link them to external conditions or species characteristics; and nor can we collect enough information to examine the features of the innovations themselves, for example, in which behavioral domains they occur or what proportion of them are cognitively complex.

The task of this article is to help resolve these problems. Our aim is to develop a definition of innovation that can serve as the basis for operational criteria for descriptive,

Grant Ramsey is Assistant Professor of Philosophy at the University of Notre Dame. He earned his Ph.D. in Philosophy from Duke University in 2007. His research is centered in the philosophy of biology. He has worked on conceptual issues in evolutionary and behavioral biology, including such topics as selection, fitness, drift, altruism, and culture. His recently published articles include "The Fundamental Constraint on the evolution of culture" in the journal Biology \& Philosophy and "Block Fitness" in the journal Studies in History and Philosophy of Biological and Biomedical Sciences.

Meredith L. Bastian, Ph.D. candidate and National Science Foundation Graduate Research Fellow in Biological Anthropology and Anatomy at Duke University, has authored publications in primate life history, alloparental infant care, and infant development. She graduated magna cum laude from Bryn Mawr College in 2000 with a B.A. in Anthropology (with honors) and Psychology. Ms. Bastian received three Intramural Research Training Awards and a Pre-Doctoral Research Fellowship from the National Institutes of Health. Funded by NSF, L.S.B. Leakey Foundation, Wenner-Gren Foundation, American Society of Primatologists, and Aleane Webb, she recently completed her fieldwork with wild orangutans in Indonesian Borneo and is currently writing her dissertation.

Carel van Schaik is Director of the Anthropological Institute and Museum at the University of Zurich, Switzerland. He earned his Ph.D. at the University of Utrecht, Netherlands, in 1985 and, after a period as postdoctoral scholar at Princeton University and research fellow at the Royal Netherlands Academy of Sciences, joined the faculty at Duke University as a professor of Biological Anthropology. His main interest is social and cognitive evolution in primates. He has studied the behavior of macaques, capuchins, leaf monkeys and orangutans in the wild, and of macaques in captivity. His recent work focuses on contributing toward explaining the origins of uniquely derived human behaviors. In particular, he studies the degree to which skills and signals in wild apes are socially acquired. cross-sectional field studies. To accomplish this we develop an individual-level process definition of innovation, and from this we derive a product definition that can be used to generate operational criteria for recognizing innovations in the field. A parallel paper (van Schaik et al. 2006) applies these operational criteria to a set of possible innovations generated in a field study of wild Bornean orangutans, Pongo pygmaeus wurmbii (see also sect. 4.5).

\section{Why study innovation?}

In order to underscore the importance of having a procedure to identify innovations in nature and to motivate the following argument, we will begin by briefly discussing some of the connections between innovation and ecology, evolution, culture, and intelligence.

\subsection{Innovation and ecology}

Innovation is likely to play a role in many aspects of a species' ecology (Giraldeau et al. 1994; Sol 2003). For example, being innovative and larger-brained increases a species' probability of surviving its release into a foreign habitat (Sol et al. 2005a). Similarly, the more innovative a species is, the better it may be at surviving an invasion by another species. At the level of the community, this would seem to imply that innovations have a positive effect on local species richness, that is, alpha diversity (Whittaker 1972). On the other hand, the innovativeness of a species is likely to be positively correlated with its niche breadth, and thus could have a negative impact on alpha diversity. As a result, innovation may push local diversity in both directions at once. The direction and magnitude of the net force will only be discerned through careful empirical work - and this work will only be possible if we are able to recognize innovations in the field.

\subsection{Innovation and macroevolution}

Innovation can play a role in the direction and rate of evolution. It has been proposed, for example, that more innovative species will tend to have higher rates of evolution (Sol 2003; Wyles et al. 1983). This may be the case, and the techniques proposed here will help in testing this prediction more rigorously. However, we suspect that innovation is unlikely to play a unitary role in evolution. Rather, the impact that innovation has on the direction and rate of the evolution of a species is probably a function of the frequency and character of the innovations produced, as well as other factors, such as the degree of social learning and adoption decisions employed by individuals confronted with new innovations. For example, it is also possible that innovative species might be able to decrease their rate of evolution through, for example, the production of niche constructing innovations (Odling-Smee et al. 2003). These innovations could help insulate the species from environmental changes, reducing the selection pressure and (thus) the rate of evolution.

\subsection{Innovation and culture}

A complete understanding of culture requires an understanding of innovation. Because only a subset of innovations becomes cultural tradition, in order to properly understand the phenomenon of culture, we must answer two questions: 
Ramsey et al.: Animal innovation defined and operationalized

(1) Why do certain kinds of behavioral novelty stick and become a permanent part of an individual's repertoire, that is, why do certain improvisations become innovations? (see sect. 3.1 for a discussion of the improvisationinnovation distinction); and (2) Why do certain innovations become culturally entrenched (we will call these cultural innovations) whereas others expire with (or before) the death of the innovator (we will call these personal innovations)? (see sect. 3.3 for definitions of personal and cultural innovations). If researchers have an operational criterion to distinguish between innovations that are passed on to at least some other individuals in the population and those that are not, then for any given population the proportion of personal and cultural innovations can be calculated. A study of orangutans found that populations differed considerably in the percentage of innovations that failed to spread (van Schaik 2003; van Schaik et al. 2003a). It is probable that transmission conditions affect the probability of extinction of innovations.

Thus, data on multiple populations allow us to ask whether the probability that an innovation transitioning from personal to cultural affects the standing innovation repertoire in a population. Reader (personal communication) found that of 606 cases of innovation in nonhuman primates, only $16 \%$ had spread to at least one other individual. The reason for this low percentage is unknown, but it may be the case that the innovations are rarely adaptive (i.e., an improvement over existing behaviors) or because the innovators are often of low status and are not closely attended to (Reader \& Laland 2001). The first systematic data from wild orangutans suggest that the probability that an innovation reaches cultural status is affected by how often and how long it is performed, assuming that the identification process is unbiased (van Schaik et al. 2006). Moreover, innovations across orangutan populations that involve individual comfort skills were less likely to spread than those concerning subsistence or communication - the latter two categories perhaps being intrinsically more salient to other orangutans. Thus, salience to observers plays a role, similar to the fate of human innovations (Rogers 1983).

\subsection{Innovation and intelligence}

Innovation and intelligence are closely related concepts. Indeed, many definitions of animal intelligence refer to novel solutions to old or new problems (Byrne 1995; Rumbaugh \& Washburn 2003; Yoerg 2001), suggesting that the ability to produce innovations is an important yardstick of intelligence (cf. van Schaik \& Pradhan 2003). Support for this contention is that reported innovation rates and learning ability are correlated among birds and primates (Lefebvre et al. 2004). Likewise, species differences in the tendency to innovate or cope with environmental change correlate strongly with the relative size of brain structures implicated in intelligence (Lefebvre 2000; Lefebvre et al. 1997; Nicolakakis \& Lefebvre 2000; Reader \& Laland 2002; Sol et al. 2005a). These studies, however, had to rely on the (often origins-based) definitions of innovations used by the authors of original reports. Moreover, laboratory studies of intelligence cannot serve to reveal the role of intelligence in the natural lives of animals because most of the procedures used to estimate intelligence in animals have extremely low ecological validity (cf. Deaner et al. 2006). We hope that the procedures supplied here for identifying innovations in the field will prove insightful in understanding the character, function, and evolution of intelligence in a wide range of taxa.

\section{Innovation defined}

The term "innovation" is used in multiple ways. It can refer to products, such as tools or behaviors, or to the processes by which these products are created (Reader \& Laland 2003a). Innovation can be considered at the level of the individual or at higher levels, such as a group, population, or species. In order to operationalize innovation and show how innovations can be detected in the wild, we require a concept of innovation that is connected to higher-level patterns. To do this, we must clearly distinguish the process of innovation from the product created by this process. Building on recent attempts to define innovation (e.g., Reader \& Laland 2003a), we draw a sharp distinction between what innovation is and how we recognize it in practice (its epistemic or operational definition). To avoid conflating the concept of innovation and its operationalization, we will first focus on innovation in the sense of the individuallevel process, because this forms the foundation for innovations as products. We will later turn to the question of how to operationalize innovation.

We define innovation (sensu process) as follows: Innovation is the process that generates in an individual a novel learned behavior that is not simply a consequence of social learning or environmental induction. (We use the phrase "innovation sensu process" to refer to the process of innovation and "sensu product" to refer to the products generated by this process.) A behavior is novel for an individual if it has never before been exhibited by that individual. Thus, an individual's behavior can be novel even if others in the population have previously exhibited the behavior. In the operationalization section we discuss the problem of determining when a behavior should be considered novel. In the following section we will discuss what we mean by learned behaviors and support our claim that innovations must be learned. We then turn to the problem of distinguishing innovations from behavior resulting from environmental induction or social learning. The three subsets of novel learned behavior - innovation, environmental induction, and social learning - are not meant to be discrete and mutually exclusive, but instead represent endpoints on a continuum, as illustrated in Figure 1. As a result, where the line is drawn between innovative and non-innovative behavior will always be to some extent arbitrary, but explicit and consistently applied criteria will make it possible to compare populations and species.

Other authors have developed definitions similar to ours, although they do not always use the term innovation (see Table 1). "Emergents," as defined by Rumbaugh et al. (1996) (see also Rumbaugh \& Washburn 2003), are innovations, as are the "acquired specializations" described by van Schaik and Pradhan (2003). Additionally, many tests of cognitive abilities of animals use the presence of innovations (new solutions to problems posed by the experimenter) as the yardstick of intelligence or cognitive abilities (e.g., Byrne 1995; Johnson et al. 2002; Yoerg 2001). For Kummer and Goodall (1985, p. 205), "[a]n innovation can be either a solution 
Ramsey et al.: Animal innovation defined and operationalized

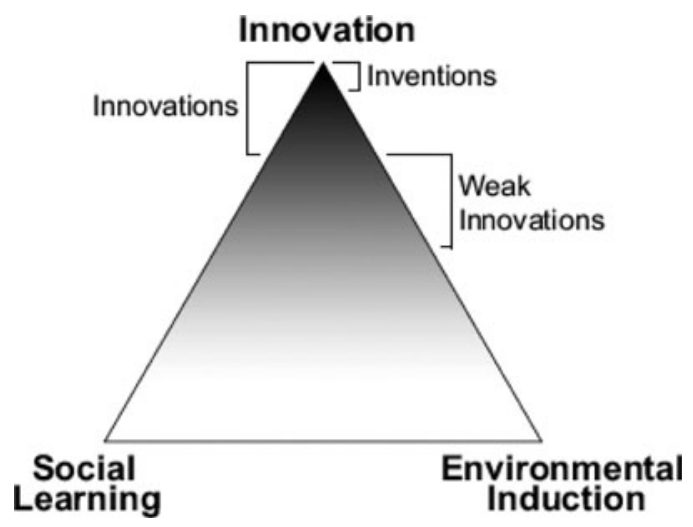

Figure 1. Novel learned behaviors may arise through three kinds of processes: innovation, social learning, and environmental induction. Instead of being discrete, these processes exist along a continuum, as represented by this triangle. Innovations are the behaviors near the apex of the triangle. Inventions are the subset of innovations closest to the apex. Weak innovations are the behaviors that are chiefly explained by the process of innovation, but also have a significant component resulting from social learning or environmental induction.

to a novel problem or a novel solution to an old one..." where "novel" is population-level novelty. This definition of innovation is similar to that of Reader and Laland (2003a), except that Reader and Laland insist that the novelty is learned. We agree with Reader and Laland that innovations must be learned, but tying the definition to population-level novelty may hinder the effective estimation of innovation repertoires and make the recognition of innovation unduly dependent on population size.

Invention is often distinguished from innovation (Janik \& Slater 2000; Simonton 2003; Slater \& Lachlan 2003). One way of making this distinction is to define an invention as "a behavior pattern that is totally novel, not obviously derived from one that an animal has been exposed to," whereas innovations are "new behavior patterns derived by modifications of previous ones" (Slater \& Lachlan
2003, p. 117). Alternatively, one might make the distinction by defining inventions as the creation of new ends, whereas innovations are simply novel ways of obtaining the same end. A problem with both ways of drawing the distinction is that it renders invention and innovation discrete, mutually exclusive categories. Because we feel inventions do not differ in kind from innovations, inventions are perhaps best considered to be a subset of innovation. Specifically, inventions can be seen as innovations that are near the pinnacle of the triangle represented by Figure 1. Relative to other innovations, inventions tend to be more rare, more novel, and involve more cognition. At the other end of the innovation gradient are weak innovations (see Fig. 1). Weak innovations are behaviors that are partially (but not fully) accounted for by social learning or environmental induction. We will return to the question of the cognitive basis for innovation in the discussion.

\subsection{Learning}

There are two central meanings of the term "learn." It can mean not a result of development or maturation and it can mean modifying the (brain of the) organism so that it behaves differently in the future. Accordingly, there are two reasons that our definition of innovation includes the restriction that innovations must be learned, which correspond to these two meanings of "learn." The first reason is that we wish to exclude novelty that is simply a result of maturation. We acknowledge that the innate-acquired distinction represents a false dichotomy: Behaviors are never simply a result of maturation (innate) or learning (acquired). Rather, behaviors exist along a continuum in which the influence of internal (genetic) or external inputs varies in strength. Accordingly, ethologists have traditionally recognized a predictability gradient of learning outcomes (e.g., Eibl-Eibesfeldt 1975; Hinde 1970), and this gradient is of great use in delineating innovation.

At one extreme, we find highly prepared learning that leads consistently to novel behaviors upon exposure to stimuli, and generally does not rely on elaborate cognitive processes (Garcia \& Koelling 1966). This extreme includes

Table 1. A list of key definitions of "innovation" and related terms

\begin{tabular}{|c|c|c|}
\hline Term used & Authors & Definition \\
\hline Innovation & $\begin{array}{l}\text { Ramsey, Bastian \& van Schaik } \\
\text { (target article: Primary } \\
\text { definition) }\end{array}$ & $\begin{array}{l}\text { The process that generates in an individual a novel learned behavior } \\
\text { that is not simply a consequence of social learning or environmental } \\
\text { induction. }\end{array}$ \\
\hline Innovation & $\begin{array}{l}\text { Ramsey, Bastian \& van Schaik } \\
\quad \text { (target article: Secondary } \\
\text { definition) }\end{array}$ & $\begin{array}{l}\text { Repertoire modification involving the addition of a new behavior, or the } \\
\text { modification of an old one, underdetermined by maturation, the } \\
\text { environment, and the behavior of conspecifics. }\end{array}$ \\
\hline Innovation & Kummer \& Goodall (1985, p. 205) & "a solution to a novel problem or a novel solution to an old one" \\
\hline Innovation & Laland \& Reader (2003a, p. 14) & $\begin{array}{l}\text { "a process that results in new or modified learned behaviour and that } \\
\text { introduces novel behavioural variants into a population's repertoire." }\end{array}$ \\
\hline Innovation & $\begin{array}{l}\text { Rogers (1983, p. 11) (Human } \\
\text { innovation) }\end{array}$ & $\begin{array}{l}\text { "an idea, practice, or object that is perceived as new by an individual or } \\
\text { other unit of adoption" }\end{array}$ \\
\hline $\begin{array}{l}\text { Acquired } \\
\text { specializations }\end{array}$ & $\begin{array}{l}\text { van Schaik \& Pradhan }(2003 \text {, } \\
\text { p. } 646)\end{array}$ & $\begin{array}{l}\text { "behaviors that do not develop reliably as a result of the interaction } \\
\text { between innate predispositions and environmental affordances" }\end{array}$ \\
\hline Emergents & $\begin{array}{l}\text { Rumbaugh, Washburn \& Hillix } \\
\quad(1996, \text { p. } 61)\end{array}$ & $\begin{array}{l}\text { "new competencies and/or new patterns of responding that were never } \\
\text { specifically reinforced by operations of the experimenter" }\end{array}$ \\
\hline
\end{tabular}


phenomena such as habituation, imprinting, and some operant conditioning. At the other extreme, the outcomes of learning are far more open-ended. The relevant external factors (stimulus configurations) and internal factors (cognitive abilities, history of exposure) are highly variable, and the specific outcomes therefore are bound to be less common. Insight learning provides the best example of this extreme. It is facilitated by behaviors such as exploration (sometimes referred to as latent learning) and predispositions such as neophilia, curiosity, and creativity. In sum, maturation is close to the predictable extreme of the learning gradient, whereas innovation is the kind of learned behavior found at the less predictable extreme of the gradient. This does not mean that innovations are necessarily cognitively complex. Perry and Manson (2003), for example, describe rather simple behaviors in white-faced capuchins - such as hand sniffing and body part sucking - that bear the hallmark of innovations.

The second reason for insisting that innovations are learned is that we wish to exclude novelty that is merely improvisational or accidental and, therefore, has little bearing on the future behavior of the individual. An improvisation (following Slater \& Lachlan 2003) is a novel behavior that fits all of the criteria of being an innovation except that it is not learned by the individual, that is, the occurrence of this behavior does not affect the probability of its reoccurrence. An accident (sensu process) is another source of novel behavior, one that arises unintentionally. Because the intentions of animals are often unknown, the distinction between improvisations and accidents will be difficult to make, especially in the field. For our purposes, however, this is not important, since the salient distinction is between innovations and the set of improvisations plus accidents, rather than between improvisations and accidents. Both accidents and improvisations can lead to innovations if the individual learns from the accident or improvisation (e.g., trial-and-error learning can occur in this way). However, as also argued by Reader and Laland (2003a), if no learning occurs, the behavior, though novel, should not be considered an innovation. For example, if a chimpanzee is observed eating a fruit that it has never previously consumed, then this behavior may be an innovation or an improvisation/accident. How are we to distinguish between these possibilities? If after the initial consumption of the fruit the chimp does not have an increased or decreased probability of eating the fruit (e.g., going out of its way to eat it again or to avoid eating it again), it is not an innovation. If the chimp subsequently habitually goes out of its way to eat the fruit, then eating the fruit is an innovation. If it subsequently avoids a fruit, then it has also innovated, the innovation being one of avoidance. (These avoidance innovations will be virtually impossible to detect in the field.)

\subsection{Innovations as products}

In addition to defining the process of innovation, we need a definition of innovation sensu product in order to operationalize innovation: An innovation (sensu product) is any learned behavioral variant created through the process of innovation. It may seem redundant to include "learned" in this definition, as learning is already a part of the definition of the process of innovation. But we do so to exclude behaviors that arose through the process of innovation (and thus were at one time learned) but have, over evolutionary time, become innate. (By "innate" we mean behaviors expected of all individuals and not resulting from social learning.) The Baldwin effect (Baldwin 1896a) can transform innovations into innate behaviors in the following way: First, a new behavioral phenotype is created through the process of innovation. This innovation increases fitness and spreads throughout the population. Although the origin and spread of the innovation are not a result of genetic variation, when and how fast individuals learn the behavior, how much exposure to conspecific models is needed, and how well they are able to perform the behavior, are variable. Assuming that there are no countervailing effects, individuals will be selected for exhibiting the behavior early and with little exposure to models. Individuals that exhibit the behavior without exposure to any models will be favored by selection over those that require learning from conspecifics. Thus, after many generations, what began as a socially transmitted innovation can eventually become innate. (See WestEberhard 2003 for an excellent discussion of the role of phenotypic plasticity in evolution.) To what extent this process is realized in nature is an interesting empirical question (see Kenward et al. 2005; Tebbich et al. 2001), but for our purpose, these Baldwinized behaviors are excluded from the definitions and analyses.

\subsection{Other subsets of novel learned behaviors}

Innovations constitute one subset of novel learned behavior (Fig. 1). How are we to distinguish innovations from the other sources of novel learned behavior: environmental induction and social learning? A novel learned behavior is environmentally induced if, given an environmental change or novel environmental element, it emerges reliably in all or most individuals exposed to the environmental stimulus. In other words, the presence of the behavior is consistently linked to the presence or absence of some environmental factor. However, the presence of such a link in wild animals is in itself not enough to decide that a particular behavior pattern is not an innovation. Consider stone handling in Japanese macaques. It is a "behavioral propensity associated with provisioning and a sedentary lifestyle" (Huffman \& Hirata 2003, p. 287). However, because it took years for it to emerge in single individuals in some, but not all, provisioned populations, stone handling qualifies as an innovation.

Socially mediated learning (social learning for short) refers to learning by an individual resulting in part from paying attention to the behavior, or to the effect of the behavior, of a conspecific (Box 1984). Social learning may involve attention to a location (due to the presence of a conspecific), an object (which is being or has been manipulated by a conspecific), or the actual behavior of the conspecific (Giraldeau 1997). Multiple psychological mechanisms are implicated in social learning; these may or may not involve observation and replication of motor acts (e.g., Caldwell \& Whiten 2002). A novel behavior is socially learned if it emerges after and because of exposure to conspecifics or their effects on the environment.

While the individual, process-based definition of innovation excludes social learning as a source of innovation, many novel behaviors that arise through innovation may 
eventually be socially transmitted. Indeed, evidence that a behavior is acquired through social learning is often an indication that the behavior originated in the population through the process of innovation (see the following section for clarification). Thus, innovations (sensu product) can be acquired by individuals either through individual learning or by social learning.

An innovation, however, is not necessarily socially transmissible. This is because the social structure or cognitive makeup of the species, or the context in which the innovative behavior occurs, might not allow innovations to be passed on. Innovations thus fall into two classes: (1) Socially transmissible innovations - innovations maintained (or maintainable) through social transmission. (The subset of socially transmissible innovations that have been transmitted to at least one other individual we call cultural innovations.) (2) Personal innovations - defined here as innovations generated by individuals but not socially transmissible. Personal innovations have also been called idiosyncratic innovations (e.g., McGrew 2004). We prefer our term because "idiosyncratic innovations" seems to imply that the innovation is unique at the population level. This precludes the possibility of multiple individuals independently producing the same non-socially transmissible innovation.

We will now elucidate the concept of innovation with a hypothetical example.

\subsection{A hypothetical example of innovation}

Consider a chimpanzee population in an environment that includes a number of nut-producing trees (cf. Boesch \& Boesch 1981; Matsuzawa 1994). One species, Dura laboriosa, produces hard nuts that require being smashed open prior to consumption. The chimps in the population invariably use readily available branches to smash the nuts and have done so for many generations. One chimp, Eureka, begins to use a readily available stone instead of a branch to break the nuts. This method of nut breaking becomes habitual for Eureka. Subsequently, other chimpanzees that observe Eureka while she breaks the nuts with a stone begin to break nuts with stones themselves. After several generations the entire chimp population has switched to stones as the tool for breaking D. laboriosa nuts.

Are we justified in claiming that Eureka actually innovated? The positive criterion in our definition requires that the behavior be both novel and learned. The behavior is obviously novel for Eureka, since she had never previously exhibited it. It is also learned, since Eureka's use of the stone was not expected and after her initial use of the stone the probability of its use increased dramatically. The negative criterion - that the behavior was not a result of social learning - is also met, in that there were no models from which Eureka could have learned her behavior. Her behavior was not environmentally induced, because there was no environmental change that accompanied and explained Eureka's initial production of the behavior. The stones did not, for example, suddenly become abundant near the trees.

If this example were modified in the following ways we would no longer hold that Eureka innovated: If Eureka had happened upon another female belonging to a neighboring community in which the use of stone tools is quite common, and if Eureka acquired the same behavior (using stones as tools to break nuts) through social learning from this female, then the behavior clearly did not arise in Eureka via the process of innovation. Consider another scenario: A community using branches to crack nuts was displaced by a forest fire and went through a phase where no branches were available to crack the nuts. During this phase stones were available and Eureka, along with most others, picked up this new habit of using stones. This new behavior, we would argue, was environmentally induced. It is conceivable that the community might continue using stones after returning to their previous environment replete with both branches and stones. Thus an environmentally induced behavior can subsequently be socially maintained and become an entrenched tradition.

These examples show how our individual-level process definition of innovation can connect to higher-level patterns. However, because in the wild we rarely have the kind of knowledge offered by these hypothetical examples, we need a method of distinguishing innovative behaviors without full knowledge of the circumstances of their origination. In the operationalization section (sect. 4) we propose a procedure for deciding whether a behavior should be considered an innovation based on the kind of partial knowledge a field biologist might possess. Before we turn to the operationalization section, we will introduce an alternative way of identifying innovation based on the notion of the behavioral repertoire - that will help clarify the distinction we have drawn between innovative, improvisational, and environmentally induced behavior.

\subsection{Repertoire flexibility and behavioral flexibility}

Organisms can be behaviorally flexible at two levels. They can exhibit flexibility at the level of their behavioral repertoire or flexibility of the behaviors themselves. Flexibility at the repertoire level occurs when an organism's repertoire is modified by either adding or expunging a behavior, or when it modifies one of the existing behaviors in its repertoire. The addition of the stone hammering behavior counts as a change in Eureka's repertoire - such a change is an example of repertoire flexibility.

By flexibility at the level of behavior we mean something quite different. A behavior can be inflexible, where each stage of the behavior follows the previous one in a stereotyped sequence, as in the classic fixed action pattern of ethology (e.g., Eibl-Eibesfeldt 1975). Alternatively, the behaviors can be flexible, in that they can be contingent upon and modified by the context in which they are executed. Thus, inflexible behavior involves a cascade of events in which the end is determined by the beginning. Flexible behavior, on the other hand, involves a branching sequence, where the end is determined by the beginning plus the inputs along the way. If Eureka gives each nut - regardless of size or form - a single hit when she uses her stone to smash nuts, her hammering would be inflexible. If, on the other hand, she inspected the nut before smashing it and smashed the large or thick-hulled nuts with greater force or more repetitions, her nut smashing behavior would be flexible.

These levels are independent: An organism can have a fixed repertoire, where the behaviors in the repertoire 
are flexible. Similarly, an organism can have a flexible repertoire, in that it can add, subtract, or modify its repertoire, but have the behaviors in its repertoire be stereotyped. For example, the chimpanzee that learns to smash nuts with stones, but smashes in a very stereotyped fashion, is exhibiting repertoire-level flexibility without behavior-level flexibility.

This distinction between repertoire flexibility and behavioral flexibility is important because innovation, as we have defined it, occurs at the level of repertoire flexibility, not behavioral flexibility. In order for an innovation to occur, it is not sufficient that a novel behavioral performance takes place - mere behavioral flexibility can do this. What is required is that the repertoire itself is modified. An innovative organism, then, is one that has a propensity to modify its behavioral repertoire. Recall that we defined improvisation as the production of unlearned novelty that is not socially learned or environmentally induced. An improvisational organism, then, is one that has a propensity to behave flexibly. Improvisation is flexibility at the level of behavior, not repertoire. An improvisational organism may or may not be innovative and an innovative organism may or may not be improvisational - each involves flexibility at a different level. It may be that the innovative and improvisational propensities are correlated in nature - organisms that are improvisational may be more likely to be innovative. This is an interesting empirical point well worth investigating. But the point here is that there need not be a link: It is at least conceptually possible for an organism to be improvisational without being innovative, and vice versa.

The modification of a behavioral repertoire is necessary for innovation to occur, but is it sufficient? No, repertoires can be modified in such a way that the modification is a response to, and determined by, the environment: Given some novel environmental element, the novel behavior is expected of all or most individuals of that type. This is what we have labeled "environmentally induced novel behavior." Thus, environmental induction is repertoire modification that is environmentally determined. (Recall the example of the chimps being forced by the fire to employ stones to break nuts.) Similarly, a repertoire can be modified in a predictable way by other individuals through social learning. Innovation thus involves repertoire modification that is underdetermined by the environment and the behavior of conspecifics. Note that by claiming that innovation cannot be determined by the environment, we are not asserting that environmental change cannot spur innovation. In fact, we recognize that many innovations involve (often anthropogenic) environmental novelty. See also the baboon example in subsection 4.2.1.

Repertoires can be predictably modified by the environment (environmental induction) or conspecifics (social learning), but they can also change in a predictable way due to maturation. Mating behavior, for example, can be absent from the young but present in the mature. (Again, this mating behavior may or may not be flexible - this is independent of the question of whether the repertoire itself is flexible.) Repertoire modification merely due to maturation is not considered innovation. Furthermore, a simple loss of a behavior from an individual's repertoire does not constitute an innovation. Innovation, then, is repertoire modification involving the addition of a new behavior, or the modification of an old one, underdetermined by maturation, the environment, and the behavior of conspecifics. As in the previous definition, "new" is new for the individual, not (necessarily) the population or species. This definition is an alternative to the definition we proposed toward the beginning of section 3 . We feel these two ways of defining innovation are equivalent: all behaviors classified as innovations by one definition should be classified as innovations by the other.

This underdetermined modification of behavioral repertoires is an individual-level phenomenon. This is what distinguishes it from the definition of innovation proposed by Reader \& Laland (2003a). Their definition is contingent upon the repertoires of other individuals in the population, making innovation require populationlevel novelty. We think that this way of defining innovation is too restrictive. Just as in the case of humans, where an individual can innovate even if another in the population already came up with the same innovation, so too in animals we feel that whether or not an innovation occurs is independent of whether others in the species or population previously or currently exhibit the innovation.

\section{Operationalization}

To say that a behavior is an innovation is to make a claim about its origin. But when we are conducting an observational study of an animal population, what we observe is a variety of behaviors exhibited in a diversity of contexts. The challenge we face is to design a procedure that indicates the likely origin of a behavior even if its inception has not been directly observed. We will do this by distilling a number of features that are characteristic of innovative behaviors.

\subsection{The operationalization of culture}

Before laying out our operationalization of innovation, we should point out that there are pre-existing methods for operationalizing culture in the wild. Because of the link between innovation and culture, we borrow some of these methods in our own operationalization of innovation. The most commonly used operational definition of culture in the wild - the geographic method - is that culture involves behaviors that are common (customary or habitual) in at least one site, but are absent in at least one other site, without concomitant genetic or environmental differences among these sites (McGrew \& Tutin 1978; van Schaik 2003; Whiten et al. 1999). This approach has been used by researchers to infer the presence of cultural behavioral variants in a variety of species (bonobos: Hohmann \& Fruth 2003; capuchins: Perry et al. 2003; cetaceans: Rendell \& Whitehead 2001; chimpanzees: Whiten et al. 1999; 2001; orangutans: van Schaik et al. 2003a). Ironically, these studies did not specify whether or not the candidate behavior patterns they investigated were innovations, even though they should all be.

The geographic method's main aim is to reduce the identification of false positives (type I errors), assuming it is applied stringently (cf. Galef 2003; van Schaik 2003; van Schaik, in press; but see Laland \& Janik 2006). However, it may be overly conservative and generate false negatives (type II errors), because it cannot recognize 
three classes of cultural behaviors: (1) cultural universals, that is, behavior patterns that are exhibited among all members of at least one age class in every observed population, the presence of which cannot be explained by maturation or environmental induction alone; (2) cultural variants that correlate with ecological or genetic differences across sites; and (3) cultural variants that for some reason are limited to small subsets within populations. The geographic method is therefore very useful to demonstrate the presence of culture (by minimizing the risk of false positives), but not optimal for estimating the local repertoire of cultural variants, that is, culture's prevalence - for the latter, the total number of false positives plus false negatives needs to be minimized.

We believe that almost all behavior patterns that satisfy the geographic method, provided it is applied stringently, are innovations, because there are no plausible alternative ways of explaining the distribution within and across sites. However, because it may fail to recognize many other behaviors as innovations, we need additional criteria for identifying innovations.

\subsection{Recognizing innovations in the field}

There are two basic approaches to recognizing innovations in wild populations. First, we can record changes in the occurrence of the behavior over time, which requires a long-term field study (or data from several field studies of the same population at different times). This is the traditional approach, and we will discuss it later. Second, we can infer whether a particular behavior is an innovation based on (1) its geographic and local prevalence and individual frequency (see previous section) and (2) properties of the behavior, such as the social role of the behavior, the context in which the behavior is exhibited, and its similarity to other behaviors. This second approach allows us to use data from cross-sectional field studies. The validity of its inferences can be checked using knowledge of spontaneous or experimentally induced behavior in captivity. We will discuss these two approaches in turn and then combine them to develop a decision procedure (see subsect. 4.4) for classifying behaviors as innovations.

4.2.1. Documenting origins. The first observed instances of a behavior in a population under long-term study in field conditions may indicate that the behavior is novel and thus potentially an innovation. This is essentially the definition put forth by Reader and Laland (2003a) and discussed in section 1 . There are two sets of conditions in which this may occur. First, a behavior might arise in an environment that was stable during the observation period. If the observers have strong reasons to believe that a behavior did not arise as a result of a long-term endogenous cycle, and if after the first appearance of the behavior it reoccurred with some regularity, it is probably an innovation.

Second, a novel behavior can arise in accordance with some change in the environment. Often, these changes are human-induced, and many of the best-known cases are of this kind: for example, sweet-potato washing and wheat sluicing by Japanese macaques (Kawamura 1959) or pecking through aluminum bottle caps to get to cream by blue tits (Hinde \& Fisher 1951). In such cases, the rate at which the behavior emerges can help to distinguish between innovations in response to novel environmental factors and environmentally induced novel behaviors. If the novel behaviors are environmentally induced, we expect many of the individuals exposed to the novel environmental element to begin exhibiting the behavior more or less simultaneously. In contrast, innovations tend to arise more rarely and the number of individuals exhibiting the behavior tends to rise more slowly, because the spread of the behavior depends, at least in part, on social learning.

It is possible for an individual to innovate using a novel environmental factor or a pre-existing novel factor that has changed in context or frequency. For instance, some savanna baboons in Amboseli, Kenya began to squeeze and drink fluids from elephant dung in the late 1980s (Susan Alberts \& Jeanne Altmann, personal communication). This is a very conspicuous behavior, yet had never been observed during many years of systematic, extensive observations of the same population, indeed many of the same individuals. It is of course possible that an ecological change such as an increased presence of elephants or the gradual desiccation of the habitat was causally related to the appearance of the behavior. However, elephant dung had been around for a very long time, and was in fact foraged on for seeds (Altmann 1998), yet had never been exploited for its moisture. Thus, if the behavior is conspicuous and if observations have continued for long enough (or comparisons with other nearby well-studied sites are possible) to exclude some annual or hyperannual cycle, then the first recorded instances of behaviors can be used as indications of innovation. However, most cases may be more ambiguous (cf. Hauser 1988). When the difference between environmentally induced novel behaviors and innovations is not easy to discern, then additional information may be needed to decide whether or not innovation has occurred.

In the case of obvious environmental change, criteria needed to decide between environmentally induced novel behavior and innovation are: (1) rarity, that is, delay between environmental change and adoption of the behavior: the shorter the delay the more likely it is that the novel behavior was environmentally induced; (2) rate of spread, from very fast to very slow: the more rapid the spread, the more likely all individuals adopted the behavior independently (cf. Reader 2004); and (3) the presence of indicators for social learning: if the path of spread in the group closely corresponds to patterns of watching, this suggests spread through social learning (cf. Henrich 2001). The more these criteria point in the same direction, the more likely the conclusion is correct.

In the case of stable environments, criteria can be: (1) rare emergence (if its rareness is not correlated with environmental factors or with social position, then it is likely to be an innovation); and (2) presence of indicators for social learning (as discussed earlier). There are, of course, degrees of rareness. The gradient from moderately rare to extremely rare correlates with the gradient from weak innovations to inventions - inventions tend to be rare, whereas weak innovations tend to be common or, if rare, are rare because of environmental factors or social position. The line used to decide how rare the emergence of a behavior has to be in order for it to be considered an innovation is a function of species-specific traits, such as 
average life span, amount of exploration, and degree of sociality.

\subsubsection{Inferring innovations from prevalence and} properties. As suggested by the field study of culture, having a snapshot of the distribution of a behavior within and between populations can help to decide whether a behavior qualifies as an innovation. We distinguish between geographic prevalence, a measure of the proportion of populations in which the behavior is recorded; local prevalence, the proportion of individuals in one site for which the behavior is recorded; and individual rate, the rate at which those individuals that possess the behavior perform it. Prevalence values can conveniently be expressed as proportions or percentages, whereas individual rates are expressed as number of occurrences per unit time.

As we have suggested, the relative values of these variables can be informative. If the behavior's geographic and local prevalence are both high (i.e., it occurs among most individuals in most populations), it is unlikely to be an innovation, unless other considerations suggest otherwise (discussed later in this article). However, if the behavior has low geographic prevalence but high local prevalence, and if presence and absence are not correlated with clear-cut genetic or environmental differences, then the behavior is almost certainly an innovation. Finally, if the behavior occurs patchily (i.e., both geographic and local prevalence are low), the distribution may still reflect a history of innovation, but it will now be more difficult to demonstrate that there are not underlying environmental or genetic differences that explain the behavioral differences.

If natural environments were homogeneous or, if in a heterogeneous environment all of the heterogeneous factors were so fine-grained that all of the individuals were expected to encounter them with the same frequency (Levins 1968), then a novel behavior that arises in an individual which is not explainable by a difference in environment or genes can confidently be considered an innovation. Natural environments, however, exhibit environmental elements in varying degrees of heterogeneity and rarity. Because of this, some behaviors that appear to be innovations because of their patchy occurrence will not, in fact, be innovations. Careful analysis is required to rule out the possibility that the appearance of a behavior may be the result of an individual encountering a rare environmental element or an unusual juxtaposition of environmental elements.

To decide whether a particular behavior that the geographic method suggests is an innovation, actually is an innovation, consideration of their properties can be a powerful tool, especially if the behavior is rare. The following is a list of the most salient properties of behaviors that can be used to make this decision.

Similarity to other behaviors. If a rare behavior is morphologically very different from all other behaviors (i.e., is composed of unusual motor acts or a highly unusual combination of motor acts), then the behavior is likely to be an innovation. For example, when an orangutan female bites into the bottom of a pitcher plant it holds up and drinks the fluid it contains, the combination of motor acts involved is unusual enough to suggest it is an innovation, especially if the behavior is also rare.
Individual attributes (status, age, sex, reproductive state, etc.). To determine whether or not a behavior is an innovation, it will be important to know the attributes of the individuals exhibiting the behavior. For example, if a behavior is rare, it will be important to know the status of the individuals exhibiting the behavior: A behavior might be rare overall, but might be ubiquitous among, and thus a characteristic of, high-ranking individuals (e.g., a particular display). At any moment in time only a single individual in the population shows this display, but every individual that comes to occupy this position will exhibit it predictably. This would make the behavior less likely to be an innovation than if it were rare but not correlated with social position. Similarly, a behavior that is rare in the population as a whole but common among pregnant females (e.g., geophagy), is less likely to be an innovation than if it were not correlated with pregnant females. Usually, both extensive data and a good knowledge of the species' natural history are required to recognize these correlates.

Context. The context in which a behavior is functional may be rare, and accordingly the behavior will be rare. But if it is not recognized as such, an observer may conclude that the behavior is an innovation. A recently published example can serve to illustrate this point. MorandFerron et al. (2004) show that a behavior of Carib grackles (Quiscalus lugubris) that is rare in the wild and is morphologically different from other behaviors - dunking, or dipping food in water prior to consumption - is nonetheless not an innovation. They conclude that the behavior is rare in the wild because of the risk of kleptoparasitism, and thus is only beneficial when performed in certain uncommon situations - for example, when there are no conspecifics close enough to steal the food. In this case, the fact that similar behavior is seen in many related species also supports the claim that dunking is not an innovation. Again, a thorough knowledge of the species' natural history is required to recognize the impact of context.

Fitness impact. If a behavior is universal (among all individuals or all individuals of a specific social position) and has a large positive fitness impact, we expect that it has at least some genetic component, because even if the behavior was initially purely cultural, it is likely that it evolved toward having an innate component (through the Baldwin effect; cf. Baldwin 1896a). Thus, if all individuals in an arboreal species sway a tree by shifting their center of gravity in subtle ways and thus make the tree bend over and connect to an adjacent tree (as in orangutans), we do not assume that this behavior is an innovation. This is a very important qualifier to the aforementioned criteria. Some behaviors may be morphologically quite distinct from the rest of the repertoire, and can be ubiquitous in part of the species' range, though not universal in the entire range. If this behavior has an obvious impact on fitness, this reduces the likelihood that it represents an innovation. This, of course, does not mean that no behaviors with a large fitness impact are innovations. The nut cracking of chimpanzees can be very important for their survival, yet most likely represents an innovation (or series of innovations), as suggested by other criteria. 
Ramsey et al.: Animal innovation defined and operationalized

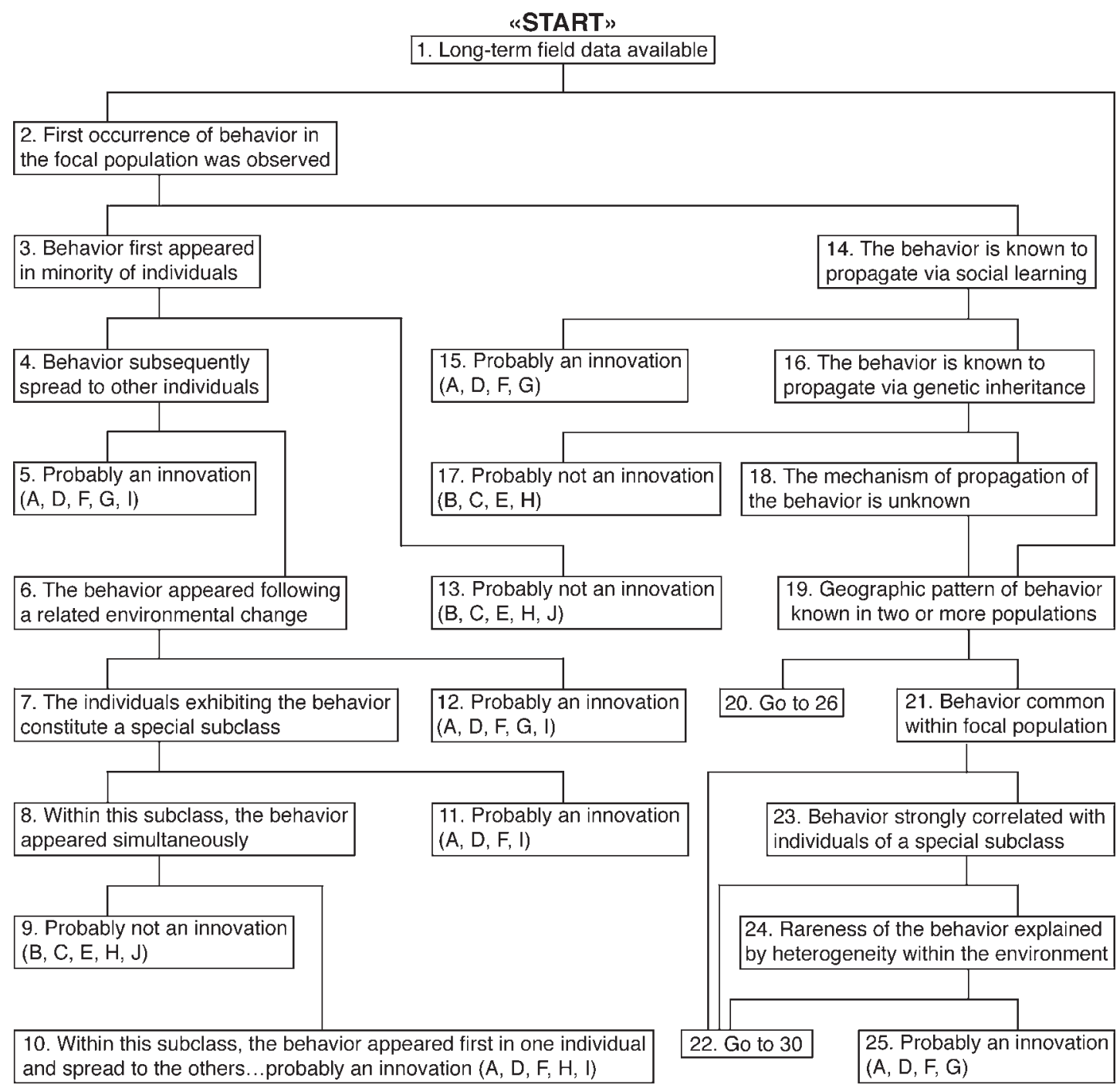

\begin{tabular}{|c|c|}
\hline $\begin{array}{l}\text { How to use this key } \\
\text { To use this key, read the statment } \\
\text { in the box. If it is true, follow the } \\
\text { line to the left. If it is false, follow } \\
\text { the line to the right. }\end{array}$ & $\begin{array}{l}\text { Criteria used to increase the probability of the conclusion } \\
\text { A. Behavior is (morphologically) similar to one or more other behaviors } \\
\text { known to be innovations (or are at least known to be socially learned). } \\
\text { B. Behavior not (morphologically) similar to any behaviors known to be } \\
\text { innovations or socially learned behaviors. } \\
\text { C. Behavior (morphologically) similar to other behaviors known to be innate. } \\
\text { D. Behavior not (morphologically) similar to other behaviors known to be innate. } \\
\text { E. Behavior exhibited in individuals raised in captivity. } \\
\text { F. Behavior not exhibited in individuals raised in captivity. } \\
\text { G. Behavior exhibited in contexts amenable to social transmission. } \\
\text { H. Behavior not exhibited in contexts amenable to social transmission. } \\
\text { I. Behavior absent from other populations. } \\
\text { J. Behavior present in other populations. }\end{array}$ \\
\hline
\end{tabular}

Figure 2. A key for determining whether a behavior should be considered an innovation.

\subsection{Comparisons with captive behavior}

Comparisons with spontaneous or experimentally induced behavior in captivity can also be very useful for deciding whether or not a particular behavior observed in the field represents an innovation. Many behaviors may seem like innovations but can be revealed to reliably emerge during ontogeny in the proper environmental context because of strong behavioral predispositions that act upon ecological affordances (Huffman \& Hirata 2003; Kenward et al. 2005; Tebbich et al. 2001). These behaviors are therefore induced or even partly instinctive; however, it is still true that the presence of role models speeds up their acquisition (Kenward et al. 2006).

As an example, consider the case of orangutans covering their heads with objects such as large leaves. This behavior is commonly observed in nature. Because it is also commonly seen in captivity with burlap bags and various other materials (Jantschke 1972), the most parsimonious interpretation is that head-covering is a behavior that is easily induced in orangutans by the presence of proper materials and that it is near-instinctive, despite its appearance of being an unusual behavior (in that it involves tool 


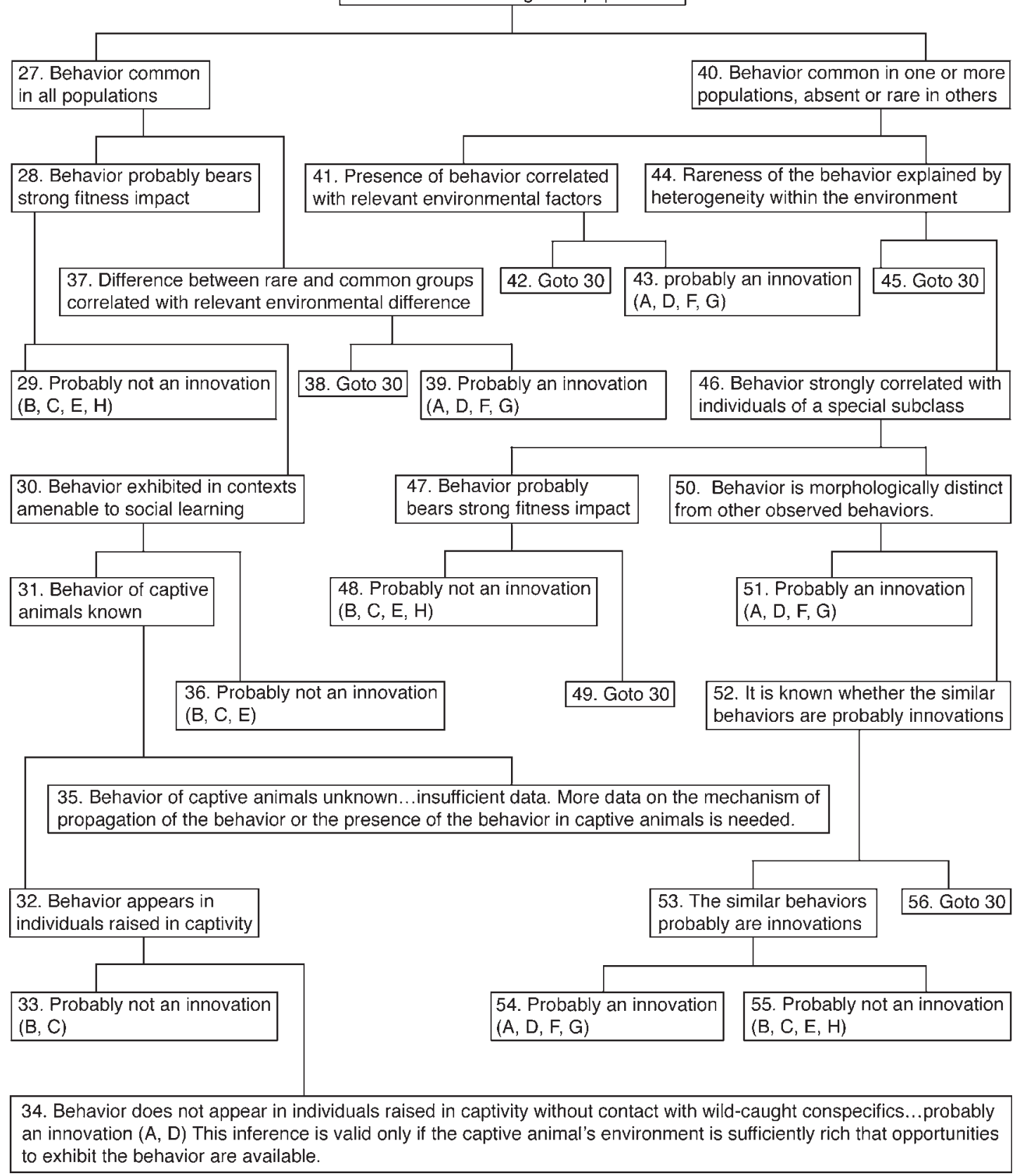

Figure 2. Continued.

use). If a behavior has a low incidence in captivity, it is more likely to be an innovation. The comparison with captive animals is facilitated by a large literature on behavior in zoos (e.g., Jantschke 1972 on orangutans; Parker et al. 1999 on gorillas).

An experimental approach can be used to help decide whether a behavior observed among wild individuals qualifies as an innovation (e.g., Boesch 1996; Morand-Ferron et al. 2004). If the behavior is an innovation that occurs in response to a particular stimulus, creating the same stimulus configuration in captivity will not reliably or rapidly yield the behavior in question. For example, if we offer an orangutan some trunks with honey-bearing holes as well as branches from which extraction tools can be fashioned, we can examine the degree to which using branch tools to extract honey from tree holes is innovative (cf. Fox et al. 1999; van Schaik et al. 1996). A problem which such experimental work will have to address, however, is that captive conditions may call forth innovations as well, and that any species is likely to produce a limited set of innovations (cf. Huffman \& Hirata 2003). To sort out this problem, comparisons across multiple captive facilities are needed.

\subsection{A dichotomous key for identifying innovations}

The key in Figure 2 can be used for determining whether or not behaviors are innovations. This key synthesizes all of the classes of data discussed earlier that can be used to identify innovations. These include: changes in the 
occurrence of the behavior over time (requiring a longterm field study or data from several field studies of the same population at different times); the behavior's geographic and local prevalence and individual frequency; and properties of the behavior, such as the social role of the behavior, the context in which the behavior is exhibited, and its similarity to other behaviors. This key uses the same positive criteria as Reader and Laland (2003a): If there are long-term data and the origin of the behavior was observed, then one can be confidant that the behavior is an innovation. We avoid the problems with Reader and Laland's approach by denying the converse of this inference: If the behavior is an innovation, there is long-term data and the origin of the behavior was observed. Instead, if the origin of the behavior was not observed, it is still possible to classify the behavior as an innovation based on other criteria described earlier.

This key can distinguish innovations from non-innovations regardless of whether any social learning was observed and regardless of whether the first occurrence (in the population or species) of the behavior was observed. In the event that the first occurrence of the behavior was observed (3), there are four cases $(5,10$, 11 , and 12) in which, if we can eliminate social learning, we can say with some confidence that the individuals that were first observed exhibiting the innovation were the ones that innovated. There are, thus, the four cases in which innovation sensu process is identified. In the remainder of the cases that identify a behavior as an innovation, the behavior may or may not come about through the process of innovation in the observed individuals.

In three cases $(7,23$, and 46) we refer to a "special subclass" of individuals. By this we mean a portion of the population that is relatively homogeneous, but distinct from the rest of the group. For example, high-ranking males or pregnant females could count as a special subclass. A behavior might be rare overall, but might be habitual among individuals of that subclass. Because rareness is a factor in identifying innovations, it is rareness within the subclass that is important, not overall rareness.

The capital letters following the prognosis ( probably an innovation or probably not an innovation) correspond to the list of corroborating evidence following the key. Once the behavior is keyed out, this list of corroborating evidence should be consulted to add support to the prognosis. The more the listed corroborating evidence accords with the data, the higher the probability is of the truth of the prognosis. The key assumes that the behaviors are not improvisations or accidents (i.e., individuals that exhibit the behavior exhibit it with some regularity).

By long term (1) we mean longer than the mean latency of the behaviors. Thus, what counts as long term will be a function of the species' characteristics. If the observations are long term, then the probability is high that the majority of the behaviors in the repertoires of the individuals in the population have been observed. Ideally, total observation time should exceed the typical life span of individuals of the species and should include observations recorded during annual (or hyper-annual) cycles.

By first occurrence (2) we do not require that the observer record the very first time that a particular behavior was exhibited. Instead, what is required is that in a population for which long-term data exist, a new behavior is seen to emerge. For example, in the baboon example in subsection 4.2.1, a new behavior is observed: drinking fluids from elephant dung. This observation counts as a "first occurrence" not because it registers the very first time that any individual in the population squeezed elephant dung, but because the behavior was first observed only after years of observation of the population.

Parts of the key are less operational than others. For example, we do not specify what sorts of measurements are required or what operations should be performed to decide whether or not a behavior probably bears a strong fitness impact (28 and 47). In such cases, one needs to rely on background knowledge for how to operationalize such concepts.

\subsection{A worked example: Wild orangutans}

The method described here was applied to a population of wild Bornean orangutans at Tuanan (van Schaik et al. 2006). Because observations on this population had only recently started, we used the cross-sectional approach, made possible by several comparative studies. Innovations were recognized based on (1) the incomplete geographic prevalence of the behavior, (2) identified causes of its absence in a population or an individual, and (3) nonsystematic comparison with the extensive studies of the behavior of captive orangutans and unpublished observations of the authors. Using this procedure, we recognized 19 clear or probable innovations at Tuanan and 43 for orangutans in general (based on absences at Tuanan but reports from other sites), some with more confidence than others. To illustrate the use of the key (Fig. 2), we will describe two behaviors from van Schaik et al. (2006) - one we consider an innovation and another we consider not to be an innovation - showing the path through the key.

Tree-hole tool-use. This behavior is defined as using a tool to poke into tree holes to obtain social insects or their products. The path followed in keying this behavior is: $1,19,20,26,40,41,43$. Because the data are not considered long term due to the long generation times of orangutans, we followed 19 instead of 2 , which leads us directly to 20 and then to 26 . Because this behavior has been observed customarily at one site, but not at any other wild orangutan sites, we followed 40 instead of 27 and 41 instead of 44 . Finally, because this behavior is only weakly correlated with known relevant environmental factors (Fox et al. 2004), we concluded that this behavior is an innovation (43).

Roof on nest in rain. This behavior is defined as a cover on the nest made by weaving together several leafy branches, which is not attached to the nest but lies loosely on top of the animal. The path followed in keying this behavior is: $1,19,20,26,27,28,29$. The path was the same as tree-hole tool-use through 26. Because the behavior is common to all studied populations and probably bears a strong fitness impact, the subsequent path was 27, 28, 29.

The results of this study of orangutan innovations are preliminary. First, we may have underestimated the innovation repertoire: It might be that particular innovations are systematically missed. This can happen if certain innovations are exhibited only rarely (true negatives) during field studies of limited duration, or if they were simply overlooked. An example of the latter may be the inclusion of certain food items in the diet. Ape diets are very broad 
in rain forest conditions, and it is not clear whether food choice is subject to innovation and social learning, although it is increasingly clear that many feeding techniques, especially those involving tools, are (van Schaik, in press; Whiten \& van Schaik 2007). Similarly, vocalizations may show subtle but systematic geographic variation dependent on social (vocal) learning. Second, we may have overestimated the innovation repertoire. The geographic information may be incomplete because a particular behavior pattern has not been recorded yet at some sites, giving the false impression of patchy geographic distribution. This may happen if the ecology or demography at these sites is subtly different, invalidating the comparison, or if observers have simply overlooked the behavior so far. We do not know the relative magnitude of these two opposing biases.

Assessments of innovation repertoires are also preliminary because they are likely to vary over time. Comparisons with captive settings turn false negatives (see sect. 4.3) into positives (or the other way around) and new innovations may arise during the study, or known behavioral variants may be recognized as innovations because sites are added in the comparisons and behaviors are absent at those new sites without explanation.

It is impossible to "prove a null hypothesis," so demonstrating something by excluding all plausible alternatives is always error-prone, and some assignments are likely to change in light of further observations. Therefore, it is the overall pattern rather than each individual item on the list that provides the most reliable information and, in this respect, the results are encouraging because they are consistent with our knowledge of cultural processes, such as which purported innovations are salient enough to reach cultural status. Furthermore, the recorded distribution of innovations across domains (subsistence, comfort, and signaling) is very similar for chimpanzees and orangutans. These patterns suggest that we captured many innovations. Finally, it is worth repeating that the approach is testable. Although we were lucky that orangutans are rather well studied in captivity, more systematic comparisons with captivity provide an independent way to verify the approach developed here.

\section{Discussion}

In this article we have offered a new way of defining innovation and added a new technique to recognize innovations in the wild. Unlike the traditional criterion of first occurrence in a population subject to long-term study (Reader \& Laland 2003a), the new technique does not require that we witness the first instance of a novel behavior. This is a major benefit because these first instances are rare, require long-term field studies, and do not provide estimates of the repertoire of innovations in a population or species. Our analysis suggests that the geographic method, as developed for operationally defining culture in nature (which requires imperfect geographic prevalence not linked to ecological or genetic variation), will also reliably identify innovations, especially when linked to comparisons with observations or experiments in captivity. For behaviors that are rare throughout the range, additional information concerning their properties may be helpful, but requires considerable knowledge of their natural history. Especially in the case of rare behaviors, comparisons with captivity may be very useful to reach a decision. The comparison with captivity can also be used to evaluate the correctness of the classifications.

The first technique (first occurrence) and the three main criteria of the second technique (patchy geographic distribution, properties of the behavior pattern itself, and incidence in captivity) can be applied systematically to examine the probability that a particular behavior is an innovation. We developed the dichotomous key presented in Figure 2 to facilitate the decision process determining whether a particular behavior is an innovation. It first asks whether long-term data are available, then it asks about the geographic distribution. When both sources of information are lacking, the probability of reliable identification of innovations decreases. The orangutan study suggests that the method can be applied (and is open to further validation), albeit only in species with substantial information on the geographic distribution of behavior patterns.

Perhaps the main weakness of the new operationalization is that it requires either data from multiple populations or long-term data from at least one population. In the absence of such data, reliable recognition of innovations will be very difficult. This requirement eliminates many species from consideration. In the species that remain, there is some concern over the standardization of the quality of observations at multiple sites, but the correctness of the classification can be evaluated by reciprocal site visits, and by comparison with captive (or ecologically naïve reintroduced) animals. We recognize that this technique may suffer from hidden biases, but we hope it is an improvement from merely relying on the first occurrence in a population under long-term observation, and especially from relying on subjective judgments as to the novelty of the behavior.

\subsection{Delineating behaviors}

A problem that arises in any study of animal behavior is the delineation of behaviors. In order to classify a behavior as novel, we must first be able to say when one behavior is different from another. At a very fine grain of analysis, each performance of a behavior represents a novel behavioral type. On the other hand, a rather coarse analysis places quite disparate behaviors under the same rubric. (Skinner 1935 makes the same point about the problem of individuating stimuli and responses.) The challenge we face when conducting field studies is to find the right grain of analysis. Sometimes this will be easy, as when the behavior is very stereotyped and not similar to any others. It is more difficult to construct a behavioral taxonomy when the behavior is not so stereotyped and varies in one or more ways (e.g., the various object combinations used in chimpanzee nut cracking; Whiten et al. 1999). It is important not to allow our preconceived taxonomies to dictate the way behaviors are classified. Rather, we should always try to get a sense of what variation is functionally relevant to the animals.

To take an example from the orangutans, they may enhance kiss-squeaks (sounds produced by sucking in breath through pursed lips) by kissing on the palm of a hand, the back of a hand, the fist, a finger tip stuck into the mouth, a wrist, leaves, a stick, or a tree trunk. Kisssqueaks on materials like leaves, tree trunks, and sticks 
are clearly morphologically different from each other and from kiss-squeaks using hands (and this can be checked experimentally in captive animals). However, are the various ways of making kiss-squeaks using hands sufficiently different that they should be considered innovations? The key is to ask whether the different forms are functionally different from the orangutan's perspective. First, we can determine whether the animals discriminate among these forms. If we find the different forms in different contexts, rather than used interchangeably in single bouts of kiss-squeaking, then distinguishing them seems justified. A second way to examine functional similarity is to examine the distribution across individuals. If multiple variants are all clustered in the same set of individuals (who also use them in an apparently indiscriminate manner) but are not used at all by others, then they are best considered a single innovation. On the other hand, if these variants are used by different individuals in different conditions, then they are best considered separate innovations.

\subsection{Innovation and cognition}

This article does not define innovation in terms of a particular psychological mechanism or class of psychological mechanisms. Instead, we have proposed a broad definition of innovation, focusing on general constraints, such as the fact that the behavior must be learned and that it must be novel. This generality might be a disappointment for some psychologists or psychology-focused biologists and anthropologists. Instead of being a defect of our approach, we feel that this is instead a benefit. The reason is that we leave it as an open empirical question, what sorts of cognitive mechanisms are required for innovation. Questions of the following kind can (and should) be answered: What cognitive mechanisms lead to the behavioral novelty in innovations? What proportion of innovations come about through accidents as opposed to deliberate foresight or insight? We have provided only a general criterion for what something has to be for it to count as an instance of innovation. We hope that this will bolster and spur both theoretical and empirical research into the basis of innovative behavior.

The main constituent psychological processes of innovation are: response to novelty, exploration (the only important component in the absence of any environmental change), and the ability to recognize a novel solution and hence repeat it. The factors affecting these underlying processes are both external to the organism, such as food scarcity, risk, and perhaps mobility (leading to "novelty" when one returns to a location after an absence), and internal, such as personality, species membership, experience (already available cognitive skills), and age, sex, and social status (cf. Reader \& Laland 2003b). Both neophilia and exploration are likely to be costly, given that both greater neophilia and curiosity (high exploratory tendency) are found among birds that inhabit islands and other habitats with reduced predation risk (MettkeHoffmann et al. 2002).

Because so many factors are involved, it may be difficult to disentangle the role of cognition, but it is reasonable to expect that innovations may be cognitively quite heterogeneous. However, little is known about their cognitive basis, at least in part because we have relatively few well-studied cases of innovation. At risk of premature generalization, it is perhaps useful to distinguish between cognitively simple and cognitively complex innovations (Whiten \& van Schaik 2007). Cognitively simple innovations reflect behavioral flexibility and correspond more with weak innovations, as shown in Figure 1 - they may emerge as accidents or the product of trial and error, although they must of course still be learned (see sect. 3.1). Cognitively complex innovations reflect the presence of causal reasoning, correspond more to inventions in Figure 1, and are brought about by systematic exploration and more prevalent affordance learning, and practice. These cognitively more demanding innovations never arise by accident, simply because the motor acts involved are highly unusual, and deviate rather strongly from the rest of the motor repertoire, or the context in which these acts are normally performed. Specific cognitively complex innovations should rarely arise, and may require observational forms of social learning in order to spread, because parallel origin in others, resulting from stimulus or local enhancement, should be just as rare as the first origin, whereas observational learning allows faithful reproduction by the observer.

Among primates, cognitively complex innovations in nature, irrespective of their domain (physical or social), may largely be limited to the great apes, who are now well-established as imitators and emulators (chimpanzees: Whiten et al. 2005; orangutans: Russon \& Galdikas 1995), including in the wild (Jaeggi et al., in preparation). This seems to be the case for tool use (Whiten \& van Schaik 2007) and tactical deception (Byrne \& Corp 2004). The major exception is the sophisticated stone tool use shown by capuchin monkeys (Fragaszy et al. 2004), along with many other social innovations (Perry et al. 2003), which are as yet inconsistent with their relatively poor cognitive skills with respect to tool use (Custance et al. 1999; Visalberghi \& Limongelli 1994). Despite this curious exception, cognitively complex innovations may be critically dependent on observational learning to spread and stave off their local extinction, providing a further argument as to why they should be limited to more intelligent species.

\subsection{Innovative species}

Our method is designed to classify behaviors as innovations, partly in order to be able to determine to what extent a population or a species is innovative. If we can estimate "innovativeness" as a species' trait, we can test predictions about the evolutionary or ecological consequences of being innovative, such as the relationship between brain morphology and innovativeness (see, e.g., Lefebvre et al. 1997; Reader 2003). There are various comparisons of innovativeness based on experimental results (see Lefebvre \& Bolhuis 2003) - partly in field conditions - but it would be useful to complement these results with patterns found in the wild.

One problem in classifying a species as innovative is that the number of innovations present in a given population or a species is a function of both organism-level propensities to innovate, as well as the degree to which innovations are passed on via social learning and thus maintained in the population. If we simply take the total number of innovations recorded for the population or species, we conflate 
the propensity of individuals to innovate and the propensity of innovations to be spread and retained. Ideally, one would estimate each propensity, but field data will generally not allow one to make this separation. Within species, populations with better conditions for social transmission (cf. van Schaik et al. 2003b) may therefore show larger innovation repertoires. This will happen if rare innovations are more likely to go extinct in less sociable populations, and might lead us to falsely conclude that more sociable populations or species are more innovative. We see no simple solution to this problem. However, for interspecific comparisons the pooled innovation repertoire across all sites may be a reasonable estimate of a species' tendency to innovate. If we can assume that the innovations arising at different sites tend to be samples from the same limited pool of potential innovations, the bias resulting from varying social transmission should decrease as the number of populations compiled to characterize a species increases. Thus, the estimated total number of innovations in a species' repertoire may be an acceptable measure.

\section{ACKNOWLEDGMENT}

We thank Susan Alberts, Robert Brandon, Judith Burkart, Victoria Campbell, Charles Catania, Barbara Finlay, Barbara Hellriegel, Peter Klopfer, Simon Reader, Alexander Rosenberg, Claudia Rutte, and seven anonymous referees for reading and commenting on earlier versions of this article.

\section{Open Peer Commentary}

\section{Novelty transmittal and innovative species}

\author{
DOI: $10.1017 /$ S0140525X07002385
}

\section{Susan Cachel}

Department of Anthropology, Rutgers University, New Brunswick, NJ 08901 1414.

Cachel@rci.rutgers.edu

Abstract: Captive or experimental studies may be of greater utility than field studies in documenting novel behaviors. Social hierarchy affects the spread of innovations. Innovative species should also be found in cases where sociality is low or absent.

The authors of the target article present a new definition of innovation, and a dichotomous key, shown in Figure 2, allows researchers to decide whether innovation exists, without conducting long-term field studies. However, longitudinal field studies may still be necessary to monitor the properties of the behavior and its changes over time. Thus, it appears that the key pertains largely to species whose behavior is either already well studied under field conditions, or to species for which future fieldwork is possible. Field studies under naturalistic conditions are the focus, although they may be impossible to carry out with many species. Innovations occurring after humans have altered or disrupted the environment may be equally important, and are becoming of increasing significance. The possibility of experiments in the field or experimentation with captive animals has been downplayed, although such studies can quickly yield far more testable data than traditional field studies can. Furthermore, experiments in the field or captive studies allow a depth of examination that may never be possible with conventional field studies.

Innovation and faithful transmission of innovations are crucial components of human culture. It is therefore not surprising that humans would assume the inevitability of transmission. An innovation that is not transmitted dies aborning. However, data cited in this article for nonhuman primates indicate that the reasons for transmission failure would be a productive area of study. The data presented here found only a $16 \%$ transmission rate to at least one other individual, out of the 606 reported cases of innovations. Granted that adaptive significance, relevance, duration of performance, and status of the innovator are important factors, there must also be additional reasons for such a massive failure to spread innovations. I suggest that hierarchical social structure in nonhuman primates hinders this spread by focusing the attention of conspecifics on details of rank and dominance interactions. Novel behaviors become associated with the social persona of the individual animals who first exhibit them and so are then not recognized as adoptable by others. For example, experimental studies of tool behavior in primates often demonstrate a lack of transmission to conspecifics, in spite of the fact that they have observed the behavior, and will even benefit from it (Cachel 2006).

Innovation is a learned behavior, because an animal must be capable of recognizing novelty as well as perpetuating it. Consequently, a memory of past events and preparation for future events is necessary for the occurrence of innovation. The authors discuss the Baldwin effect, which indicates how a novel behavior can become a species-specific behavior. However, given the definition of innovation used here, is it possible for animals considered to be of lesser intelligence or of low cognitive capacity to be capable of innovation at all? Must novel behaviors in these animals arise only by accident or improvisation before they become fixed in a population? If one considers the repertoire of very complex behaviors that occur in social insects (e.g., nest or hive construction, individuals linking together to bridge a spatial gap, fungus farming), does this article imply that such behaviors, which are formed from a series of complex actions, could never be classified as innovations, because they arose as a concatenation of individual accidents or improvisations? The origins of new behavior in animals with little individual behavioral plasticity, but with possible social transmission of novelty, is an interesting theoretical question.

The target article rightly argues that innovation is fundamentally related to questions of animal culture and intelligence. The authors focus largely on higher primate examples, and emphasize the interaction of culture and innovation. That is, culture represents a compendium of innovations, but it also preserves innovations from extinction by spreading novelty through observation and social learning. An innovation that is spread widely enough is then preserved from local extinction. Practitioners of the behavior are then saved from the necessity of reinventing it.

However, one could argue that an individual capacity for innovation might be more useful in long-lived solitary animals, or animals that live in small groups. Innovative species should also be found in cases where sociality is low or absent. This is because of the likelihood that an innovation can easily be lost through the death of an individual practicing it, or the deaths of several members of a small group. In this case, individuals that were capable of innovation could invent or reinvent solutions to problems that they encounter. One would expect that these species would exhibit high levels of exploratory behavior, and would demonstrate neophilia or novelty seeking. Attributes such as curiosity and play might also be present. Otters are an example of this type of species. Among primates, orangutans exemplify these traits, because they are long-lived and fit the 
classical definition of being a solitary species. Innovative species are not necessarily species with complex sociality; sociality may, in fact, be absent. On the other hand, insect societies with complex sociality may be composed of individuals with low behavioral plasticity and yet show an ability to innovate.

\section{Behavioral innovation and phylogeography}

\section{DOI: $10.1017 /$ S0140525X07002397}

\section{Pierre Deleporte \\ UMR 6552 (Ethologie, Evolution, Ecologie Lab), CNRS, Université de Rennes, 35380 Paimpont, France. \\ pierre.deleporte@univ-rennes1.fr \\ http://www.umr6552.univ-rennes1.fr/PierreDeleporte.php}

\begin{abstract}
Indirect identification of innovations in wild populations involves inferring past, unobserved behavioral events. Such historical inference can make simple use of present distribution patterns of differently behaving individuals, but population genetic studies are a potential source of complementary relevant information. Methodological lessons can be taken from phylogeography, that is, molecular approaches to the history of population spatial distribution patterns and gene flows. Opportunities for such studies in primates should increase with the developing population genetic studies used for management and conservation purposes.
\end{abstract}

Ramsey et al. raise the general problem of diagnosing behavioral innovation in the field through indirect approaches, emphasizing the high relevance of distribution data in populations. I argue that this constitutes de facto a historical approach at the population level; hence it should be possible to take advantage of complementary historical inferences from molecular phylogeography.

The notion of innovation has an obvious historical dimension, in that it qualifies a scenario of the appearance of a novel behavioral pattern and its further diffusion within populations as being a "cultural innovation" (according to Ramsey et al.'s terminology). A standard approach for inferring or testing historical scenarios of behavioral evolution is phylogenetic inference, for example, adaptation viewed as historical novelty, as previously discussed in this journal (see Andrews et al. 2002 in BBS 25(4), and associated comments therein by Blackburn, Deleporte, and Roney \& Maestripieri). But such studies are primarily concerned with highly genetically influenced behaviors inherited in different species or higher phylogenetic taxa. Here the focus is clearly on learned behavior and relatively shorter time periods.

Closely related to this concern, molecular phylogeography involves comparing population distributional data with genetic data in order to reconstruct the relatively recent history of spatial distributions and variations in gene flows (e.g., Templeton 1998). This perspective seems clearly more appropriate for Ramsey et al.'s concerns regarding, for example, the presence of a prevalent putative cultural innovation in some, but not all, of the studied populations.

However, the authors frame the question of informative populational data in a somewhat limited way. Their notion of "geographic prevalence" is simply defined in this article in terms of overall presence/absence statistics, namely, "the proportion of populations in which the behavior is recorded" (sect. 4.2.2), without specific reference to spatial patterns. Concerning genetic data, the authors refer to a general notion of occurrence "without concomitant genetic or environmental differences" between populations (sect. 4.1). They apparently mean that the behavioral differences at stake should not be attributable to direct genetic influence, so that the behavior being considered is likely learned. Hence, they implicitly refer to the genetics of behavior rather than to the molecular data as phylogeographic markers.
Compared with simply taking into account the proportion of populations showing the putative innovation (rough "geographic prevalence"), molecular phylogeographic studies may allow for finer considerations. One can map individuals showing the putative innovations on the geographic map of population spatial locations, and also on the cladogram or network of their genetic affinities. Contrasting these two maps reveals their possible consistence and discordances. A conjunction of contiguous spatial distribution of populations containing "innovating" individuals and molecular affinities indicating sustained gene flow between the same populations, possibly down to parental affinities by using appropriate markers, would be suggestive of cultural diffusion of a unique innovation through migration of individuals contributing to both cultural diffusion and gene flow between populations. Otherwise, the strict geographic pattern of diverse rates of "local prevalence" of the behavior inside a series of contiguous populations could also suggest routes of ongoing cultural diffusion, with decreasing prevalence along spatial gradients of progression.

An important and growing concern in phylogeography is that distances in a straight line between populations may not be the best parameter for contrasting spatial distribution with genetic similarity. Hence, the developing concept of "ecological distance" is preferred to rough geometric distances as the crow flies. The general idea once again is to keep the analysis realistically "close to the map" as well as to effective ecological constraints on animal dispersal abilities, notably through possible "landscape corridors" of suitable habitat, rather than reasoning according to an ideally isotropic environment. Such considerations may usefully be included in the previous analysis, with spatial proximity being understood through suitable habitat connections rather than by using a purely geometric basis.

It seems that the possibility of cultural losses is not directly addressed by Ramsey et al., as if innovations could appear and spread, but never vanish, for example, through replacement by other behaviors, or according to environmental changes. A likely explanation is that cultural loss, however plausible, may seem very difficult to assess. Lessons in this respect can also be taken from phylogenetic scenario testing, notably by addressing the question of possible convergent novelties (convergent "autapomorphies" in phylogenetic jargon) versus repeated losses (or "reversals") of a previously widespread plesiomorphic biological character (i.e., a relatively ancient one). The problem is that resulting distribution patterns in phylogenetic clades, and here in populations, can be exactly the same in cases of multiple convergence or losses. Stated another way, the absence of a behavior can be due either to the fact that it never occurred in the population, or that it already disappeared. But the phylogenetic solution (optimizing scenarios of common descent) is hardly applicable at the population level for learned behavior because of the imperfect fit to genealogy and the importance of spatial connectivity allowing inter-population transfer of cultured individuals. Only complementary information can allow a decision for population innovations. Here, the distributional pattern of a cultural innovation in way of disappearance should generally be both spatially and genetically scattered, and hence not likely the direct result of common cultural inheritance and transfer to adjacent populations. A complementary qualitative criterion is suggested by the authors: that is, a high specificity and complexity of the behavior would suggest that convergence is not likely.

Other notions put forth in Ramsey et al.'s article could also profit from conceptual and methodological reflections in phylogeny and phylogeography, such as the importance of, and the difficulties involved in defining and delineating characters (Pogue \& Mickevich 1990), or similar problems with delineating populations (Waples \& Gaggiotti 2006). Otherwise, the question of the fit between genetic relatedness and cultural transmission of innovations could in itself be the object of investigations, as is the case in humans; an example being the study of the westward spread of both agricultural techniques and human genes on the Euro-Asiatic continent (Cavalli-Sforza et al. 1994). Time 
scale apart, such studies should be inspiring to primatologists, with a notable difference being that diagnosing cultural traits seems rather direct in humans.

It could be questioned whether the perspective of adding molecular phylogeographic analysis to investigations about innovation is feasible in practice. This is in effect a highly demanding approach, but there should be increasing opportunities for such studies, notably in primates, with the development of noninvasive population genetic studies for fundamental research and for management and conservation purposes, as well. Recent examples in two emblematic taxa include: the case for gorillas (Bergl \& Vigilant 2007; Douadi et al. 2007) and, as could be expected, orangutans (Goossens et al. 2006).

\section{ACKNOWLEDGMENT}

I thank Eric Petit for very helpful suggestions and Nanette Anderson for help in improving the English.

\section{Knowing psychological disposition might help to find innovation}

\author{
DOI: $10.1017 /$ S0140525X07002403
}

\section{Gyula K. Gajdon}

Department for Neurobiology and Cognition Research, University of Vienna, A-1090 Vienna, Austria; Konrad Lorenz Institute for Ethology, Austrian Academy of Sciences, A-1160 Vienna, Austria.

gyula.gajdon@univie.ac.at

http://www.nc.univie.ac.at/index.php?id=7246

Abstract: Ramsey et al.'s article provides a more sensitive framework for comparative innovation than others' operationalisations have done. Nevertheless, a methodology has to be elaborated in order to determine to what degree a behaviour is novel. Psychological processes have to be considered when evaluating the value of reference groups and in order to figure out where to look for innovation.

Ramsey et al. are probably right not to operationalise innovation in terms of psychological processes (as they write in sect. 5.2) in order to grasp examples from the whole spectrum of the phenomenon. Caro and Hauser (1992) did something similar when investigating teaching in animals. The impact of a particular behaviour on fitness is only indirectly linked with its mechanism. Thus, it seems appropriate to make a phenomenological inventory.

Nevertheless, I feel that we might increase our sensitivity for innovation if we elaborate on the interrelation between psychological processes and innovation. I say this, first, because despite the claim the authors make, their article is inherently about issues of psychological processes. I also say this because I think that this approach will help us to find more examples of innovation.

The target article is inherently psychological because the authors propose that innovation is mainly an intrinsically driven (yet not innately determined) process: In their Figure 1, they set innovation well apart from externally induced processes for novel learned behaviour. This view is the main difference between the operationalisation of innovation provided by Lefebvre et al. (1998) and that of the authors of the present target article. For the former authors, simply any type of behavior that was reported as being novel contributed to the innovation frequency of a species, regardless of whether it was environmentally induced or not. This approach is more consistent with regard to indifference to the mechanisms involved in innovation than the approach of Ramsey et al. It is possible in principle, for example, that the impact of a "weak" innovation on the performer's fitness is considerably larger than that of an invention.

Differentiating between varying forms of innovation is an issue of classifying different mechanisms and degrees of novel

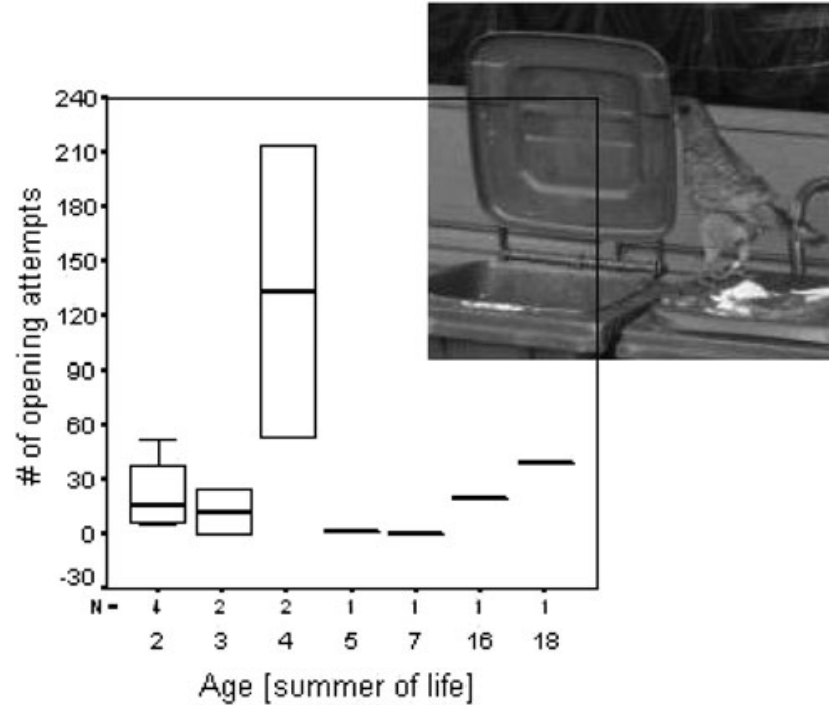

Figure 1 (Gajdon). Subadult keas in their fourth summer of life are an extraordinary subgroup with respect to their persistency in attempting to open rubbish lids. This makes them most likely to innovate solutions for mechanical problems (Gajdon et al. 2006).

behaviour with different likelihoods that the novel behaviour will be displayed. The effort that Ramsey et al. have made in differentiating between these forms will be appreciated by members of the scientific community - myself included - who feel puzzled by the indiscriminative approach by Lefebvre and his collaborators, though the latter nevertheless has yielded interesting results (Lefebvre \& Bolhuis 2003; Timmermans et al. 2000). As the authors of the target article mention, distinguishing different forms of innovation might be the first step to comparing the complexity of innovation between species. However, a more sophisticated methodology has to be elaborated in order to determine to what degree an innovation deviates from the usual ethogram of a given species, sub group, or individual, or to which degree it has to cope with novelty and by which mechanisms it does so when innovating. This might have a considerable impact on whether we consider a behaviour to be innovative or not.

For example, in Figure 2 of target article, in most boxes of the key for determining whether a behaviour is an innovation, and where there is a reference to the occurrence of the candidate behaviour in captivity, its absence in captivity increases and its presence in captivity decreases the probability that the behaviour is an innovation in the field. However, taken that Kummer and Goodall (1985) are right that some animals might be more innovative in captivity, this will be a conservative indicator for innovation in such species. For example, it was found that only a few individual keas, Nestor notabilis, an endemic mountain parrot of New Zealand, removed a tube from a long upright pole, thereby gaining access to a reward inside the tube (Gajdon et al. 2004). However, the majority of keas succeeded in the aviary. This result from captivity does not necessarily reduce the probability that the birds were innovated to remove the tube in the field. If the birds reared in captivity are more attentive to combining objects with each other, as indicated by ongoing developmental investigations, captive animals are more likely to bring the tube to the end of the pole. Thus, they might find the solution more easily because of a competence in sensorimotor intelligence that their wild conspecifics might not rely on. One approach to evaluating the referential value of captivity is to provide simple tasks in the field and laboratory to determine differences in motivation and organisation of exploratory behaviour and sensorimotor intelligence. 
The example of the kea indicates that the issue of ontogenetic development as a process for new behaviour should not be reduced to maturation. Rather, the interaction of environmental condition and cognitive development might equip animals with different skills that make them more or less likely to innovate. For example, an animal's willingness to interact with novelty is a crucial skill for innovation. Besides birds from island populations being more explorative (Haemig 1989; Mettke-Hofmann et al. 2002), Mettke-Hofmann et al. (2005a) also found that warblers of a migratory species were less explorative than warblers of a resident species, but that the difference diminishes at the beginning of the breeding season. Fairbanks and McGuire (1993) showed that the parental style of vervet monkey mothers had considerable impact on how their offspring reacted to novel situations. A subject's willingness to interact with novel situations also changes during ontogeny. In vervet monkeys, it increases in juveniles and decreases as the animals become older (Fairbanks 1993). Such studies indicate that the psychological disposition an individual displays for interacting with novelty changes during its life span, so that it will profit when changes are most likely to occur and when interacting with them is most needed (e.g., when an individual leaves its natal group site; Bischof 1985).

From this it follows that (1) each individual needs its own referential subgroup to evaluate the degree by which a novel behaviour deviates from the subjects' usual performance (see Fig. 1); (2) this should not dispute the fact that there are subgroups that are more likely to innovate; and (3) considering mechanisms that provide a disposition for innovation, as well as characteristics of the species' life history, can help to find innovation when it is most likely to occur. This might reveal a considerably different picture of innovative capacity than that which we have gained so far with previous operationalisations of innovation.

\section{Signs of culture}

DOI: $10.1017 / S 0140525 X 07002415$

\section{R. Allen Gardner \\ Department of Psychology and Center for Advanced Studies, University of Nevada, Reno, NV 89557. \\ gardner@unr.edu}

Abstract: Ramsey et al. present an ingenious method to study behavioral novelty under field conditions within relatively narrow time constraints. This evokes discussion of lateral spread of innovation from individual to individual versus vertical spread from generation to generation. This discussion of incipient culture helps to place the traditional biological tool of cross-fostering into philosophical and anthropological context.

In this thought-provoking article, Ramsey et al. develop a rationale for a practical method to study behavioral novelty under field conditions. The virtue of this operational definition is that it lends itself to field studies with relatively narrow time constraints. As happens in the best of proposals, virtues and weaknesses arise from the same source, in this case the constraints of relatively short-term field observations. The authors clearly point out this inherent weakness, but choose instead to devote discussion to details of method and positive results - as well they should. A strength of this article is that in the course of presenting an ingenious operational definition and its implications, Ramsey et al. draw attention to central issues of innovation and incipient signs of culture in nonhuman animals.

Incipient culture. Ramsey et al. discuss lateral spread of new behaviors from individual to individual within a social group much more than vertical transmission from generation to generation. Yet, transmission across generations, especially from parent to offspring, seems closer to the concept of human culture than lateral transmission. In human cases, lateral spread from individual to individual within a single generation could be fad or fashion rather than culture. Boesch-Achermann and Boesch (1993), for example, observed wild chimpanzees using tools to crack nuts. Nut cracking with tools must have originated as a novel behavior sometime in the history of this social group of chimpanzees. Implications for a kind of culture of nut cracking depend, however, on transmission across generations, particularly on observations of mothers teaching infants (Boesch 1991).

Long-term field studies tend to require monumental effort and time, but can also yield monumental results (Goodall 1986; Watanabe 1994). However, cross-generational transmission appears in laboratory experiments. Previde and Poli (1996), for example, taught a novel method of food retrieval to female hamsters. Pups observed their mothers and later incorporated the novel method into their - second generation - food retrieval.

Fostering culture. Cross-fostering - parents of one genetic stock rearing the young of a different genetic stock - is a traditional tool of behavioral biology for studying the interaction between genetic endowment and developmental environment (Immelmann \& Beer 1989; Scott 1958; Stamps 2003). Members of fostered species have adopted species-specific behavior of fostering parents, for example, migratory habits (Harris 1970) and flight and feeding habits (Rowley \& Chapman 1986). In the context of the present discussion, behaviors adopted by crossfosterlings are clearly novel to the fostered species. Their adoption across generations looks very much like culture.

Sign language studies of cross-fostered chimpanzees (Gardner et al. 1989) take the method of cross-fostering farther because chimpanzees resemble humans so closely, particularly in their comparably long childhood (Gardner \& Gardner 1989, pp. 45). Cross-fostering is very different from rearing a chimpanzee in a conventional laboratory staffed by human caretakers. Cross-fostering is also very different from keeping a chimpanzee in a home as a pet. Many people keep pets in their homes. They may treat their pets very well, and they may love them dearly, but they hardly treat them like children. Providing a nearly human infant environment all day every day for years on end is a daunting laboratory challenge (Kellogg 1968).

All aspects of intellectual growth are intimately related. For young chimpanzees, no less than for human children, familiarity with simple tools such as keys, devices such as lights, articles of clothing such as shoes, are intimately involved in learning signs or words for keys, lights, shoes, opening, entering, lighting, and lacing. The human-simulated homes of chimpanzees, Washoe, Moja, Pili, Tatu, and Dar were well-stocked with human objects and activities, and cross-fosterlings had free access to them, or at least as much access as young human children usually have. They ate human style food at a table, with cups, forks, and spoons. They helped to clear the table and wash the dishes after a meal. They used human toilets (in their own quarters and elsewhere), wiped themselves and flushed the toilet, and even asked to go to the potty during boring lessons and chores (Gardner \& Gardner 1989).

The daily language of this infant world was American Sign Language (ASL), the naturally occurring language of deaf communities in North America. English, the language of earlier studies, demands a vocal apparatus and vocal habits that seem to be beyond chimpanzees. Without conversational give-andtake in a common language, cross-fostering conditions could hardly simulate the environment of a human infant. Whenever a cross-fosterling was present all verbal communication was in ASL. Total immersion in human language as well as human culture is essential for rigorous, human cross-fostering.

A naturally occurring human language acquired in a human cultural context permitted human comparisons. Size of vocabulary, appropriate use of sentence constituents, number of utterances, proportion of phrases, and inflection all grew robustly throughout five years of cross-fostering; robustly, but more slowly than in human children. Growth was patterned growth and patterns were consistent across chimpanzees. Wherever 
there are comparable measurements, patterns of growth for cross-fostered chimpanzees paralleled in detail characteristic patterns reported for human infants (B. Gardner \& Gardner 1998; R. Gardner \& Gardner 1998). All of ASL was novel to chimpanzees, of course.

Pragmatic devices that characterize human conversation also appeared in conversations of cross-fostered chimpanzees (Bodamer \& Gardner 2002; Chalcraft \& Gardner 2005; Drumm et al. 1986; Jensvold \& Gardner 2000; Shaw 2000). Conversational pragmatics of intensity, agreement, and turn-taking seem more cultural than Chomsky's innate deep structures. Cross-fostered chimpanzees converse with each other in the absence of any human presence with only video cameras to make records (Fouts \& Fouts 1989). Project Loulis adds a further cultural dimension. The infant, Loulis, adopted by Washoe when he was about a year old, learned more than 50 signs of ASL that he could only have learned from other chimpanzees (Fouts et al. 1989). A culturally relevant laboratory succeeded where Terrace et al. (1979) failed with rigorous Skinnerian reinforcement. Human-like development depends on human cultural context. Ramsey et al. help to place a traditional biological method in philosophical and anthropological context.

\section{Can a restrictive definition lead to biases and tautologies?}

DOI: $10.1017 /$ S0140525X07002427

\section{Luc-Alain Giraldeau, ${ }^{a}$ Louis Lefebvre, ${ }^{\mathrm{b}}$ and Julie Morand- Ferron $^{\mathrm{a}}$}

${ }^{a}$ Département des Sciences Biologiques, Université du Québec à Montréal,

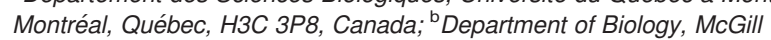
University, Montréal, Québec, H3A 1B1, Canada.

giraldeau.luc-alain@uqam.ca louis.lefebvre@mcgill.ca

morand-ferron.julie@uqam.ca

Abstract: We argue that the operational definition proposed by Ramsey et al. does not represent a significant improvement for students of innovation, because it is so restrictive that it might actually prevent the testing of hypotheses on the relationships between innovation, ecology, evolution, culture, and intelligence. To avoid tautological thinking, we need to use an operational definition that is taxonomically unbiased and neutral with respect to the hypotheses to be tested.

In the target article, Ramsey, Bastian, and van Schaik (Ramsey et al.) propose a new definition of animal innovation and a 55step key to help field workers decide whether a putative case is a true one. We applaud any attempt to improve upon the first papers in what is a very young field, but doubt that Ramsey et al.'s effort will be useful to many researchers.

Why study innovation? The authors propose four reasons for the study of innovation: ecology, macroevolution, culture, and intelligence. The ecological reasons do not require the subtle categorization provided here. In fact, behavioral diversity is blind to the origin of the innovation; it could be genetic or environmentally induced and so most ecologists will be unconcerned about its precise origin. The same holds for its macroevolutionary interest. The extent to which a species is insulated against environmental challenges depends on its ability to adapt its behavior to modified conditions, suggesting that environmental induction is a much more important factor than innovation to students of macroevolution. Ramsey et al. state that innovation can sometimes contribute to culture, but most of their article confuses the two concepts and in a somewhat circular logic, eventually considers culture as an indication of innovation. Finally, the relevance of innovation to intelligence is decreed, not demonstrated. There is no a priori reason to believe that intelligence is associated with innovativeness; that association must be the subject of empirical scrutiny, not a declaration of self-evidence.

What is an innovation? The approach to the concept of innovation that is presented here is to decide a priori on the characteristics that it should possess if it were to be recognized. This leads to a regression of finer and finer details of classification to make sure the category is as pure as possible. So, one must decide that the behavior is learned individually, not part of normal maturation, not an accident, and not induced by an environmental change. Whatever is left then is a real innovation, which can be categorized as an invention, or a weak versus a strong innovation, depending on whether the innovation results from simple or complex cognition - categories that appear entirely tautological and far from being amenable to strong quantitative study.

Is the operational definition really operational? Going through the steps of the proposed 55-step key, it becomes clear that the definition does not do away with guesswork. In fact, it makes guessing a systematic process where one must decide whether one's study falls into one or another dichotomous category, many of which can only be decided by subjective decision (e.g., "behavior common in focal population," box 21 - what exactly is "common"?). A great deal of the key requires knowing ecological properties that have posed significant measurement problems to ecologists (e.g., environmental heterogeneity). Moreover, some possibilities will be difficult to reject. For example, while it is possible to detect an environmental change responsible for the appearance of a novel behavior, the non-detection of such a change cannot be taken as meaning the absence of change. Given the potential costs of eating novel foods, entering new habitats, or trying novel foraging techniques, environmental induction is likely to be a powerful driver of innovations. We thus feel that Ramsey et al.'s exclusion of environmentally induced innovations is too restrictive. The idea behind this exclusion seems to be borrowed from the ethnographic approach to animal culture. In that context, the exclusion is relevant. However, we think more harm than good can be done with this criterion in the study of innovations.

The operational definition of innovation relies on a series of assumptions that are not all self-evident. To recognize innovations in the field, for instance, you must first accept that innovations tend to arise more rarely than environmental induction. The number of individuals exhibiting the innovation tends to rise more slowly because the spread of the behavior depends, at least in part, on social learning. Moreover, innovation requires a longer delay from the moment the environment changes than environmental induction. Here, the more rapid the spread the more likely individuals have adopted the behavior independently, because social learning is assumed to be slower than the individual learning that is involved in environmental induction. These considerations ignore that the speed of acquisition of a novel behavior also depends on costs and benefits to the behavior and its alternatives, not simply on its means of diffusion. The assumptions used by Ramsey et al. are perhaps correct, but they are far from selfevident and must be the object of scientific scrutiny. Rarity is also important in identifying an innovation, but rarity must be defined as a function of species-specific traits such as degree of sociality and the amount of exploration, clearly not easy empirical measures. The use of such subjective criteria will prevent comparisons of data from different research programs and different taxa, thereby hampering comparative work on innovativeness.

How to avoid tautologies: The use of a neutral definition. In birds, accounts of innovation were first encouraged by Thorpe $(1943 ;$ 1956), who used the terms "unusual" and "original" to qualify these innovations. In primates, Goodall's section of the pioneering paper by Kummer and Goodall (1985) defines innovation as "a solution to a novel problem, or a novel solution to an old one"; a communication 
signal "not observed in other individuals in the group"; "a new ecological discovery such as a food item not previously part of the diet of the group" (p. 205, our italics). The first comparative studies of innovations used the terms italicised here as inclusion criteria in their databases. All subsequent comparative tests of neural, cognitive, ecological, or evolutionary correlates of innovation were based on these criteria.

In order to test a scientific prediction, it is important for the definitions of the variables involved in the test to be as neutral as possible with respect to the hypothesis. If this is not the case, the result can be tautological. We believe that the many conditions required by Ramsey et al. for a behavior to be considered innovative will lead to taxonomic biases, rendering invalid many of the tests the authors cite in their section 2 to justify the importance of innovations. Ramsey et al. recognize this danger when they say that "cognitively complex innovations . may largely be limited to the great apes" (sect. 5.2, para. 4). This may very well be true, but a statement like this should be the result of an objective test, not a possible artifact of a definition. Terms like "cognitively complex" can be vague and difficult to use objectively; the emphasis on intelligence can create a circular relationship with brain size.

We believe that the descriptors initially proposed by Thorpe and Goodall and used since then by others, are much more neutral and unbiased than the criteria proposed by Ramsey et al. What precision might be gained in the study of a few cases by the application of Ramsey et al.'s criteria will be lost through the biases introduced in comparative trends. The use of neutral key words and the reliance on the judgment of the initial observers of the innovation is bound to introduce some type 1 error. However, if this error is randomly distributed and/or can be quantified and removed (as population size, research effort, observer bias and other variables have been in studies of birds and primates), valid tests of predictions can still be made. This is unlikely to be the case with Ramsey et al.'s criteria.

\section{Genetic assimilation of behaviour does not eliminate learning and innovation}

DOI: $10.1017 / S 0140525 X 07002439$

\author{
Gavin R. Hunt and Russell D. Gray \\ Department of Psychology, University of Auckland, Private Bag 92019, \\ Auckland 1142, New Zealand. \\ grhunt10@hotmail.com rd.gray@auckland.ac.nz \\ http://www.auckland.ac.nz
}

\begin{abstract}
Ramsey et al. attempt to clarify methodological issues for identifying innovative behaviour. Their effort is seriously weakened by an underlying presumption that the behavior of primates is generally learned and that of non-primates is generally "innate." This presumption is based on a poor grasp of the non-primate literature and a flawed understanding of how learned behaviour is genetically assimilated.
\end{abstract}

Ramsey et al. make a much-needed attempt to clarify methodological issues for identifying innovative behaviour in animals. However, their effort is seriously weakened by an underlying presumption in their article that the behaviour of primates is generally learned whereas that of non-primates is generally "innate." Three related reasons appear to explain this presumption: (1) There is a heavy emphasis on innovative behaviour by nonhuman primates, giving the false impression that innovative behaviour is rare in non-primates. This may reflect a continuing primatecentred view of animal cognition among many primatologists (Emery \& Clayton 2004a). (2) Potential innovations are excluded because of a flawed understanding of how learned behaviour is genetically assimilated. And (3) there is a poor grasp of the non-primate literature.
The authors exclude tool manufacture and use by New Caledonian crows (Corvus moneduloides, NC crows hereafter) as a potential source of innovations because they imply that the behaviour is "Baldwinian," or "innate." They base the exclusion on recent findings that captive naïve juvenile crows developed proficient stick tool use (Hunt et al. 2007; Kenward et al. 2005). NC crows (and woodpecker finches Cactospiza pallida; Tebbich et al. 2001) clearly have a strong predisposition for basic tool use. However, Ramsey et al. confuse the basic tool skills of naïve juveniles with the considerably more accomplished skills of free-living adult NC crows. NC crows manufacture a diverse range of tools that include hooked tools made from sticks and similar material and distinct designs (wide, narrow, and stepped) made from the leaves of Pandanus species trees (Hunt 1996; Hunt \& Gray 2002; 2003; 2004). Although all the naïve crows mentioned ripped Pandanus leaves placed in their cages, only one bird made and used "rough" Pandanus tools. The shapes of these crude tools showed little resemblance to any of the three standardized shapes produced in the wild. The obvious mismatch between the basic tool skills of the naïve juveniles and the more complex skills of experienced free-living $\mathrm{NC}$ crows suggests that the tool skills used in the wild require learning. Four lines of evidence indicate that learning is involved in the development, maintenance, and evolution of NC crows' tool skills in the wild: flexibility, different tool designs, innovative behaviour, and a prolonged learning period in juveniles.

Behavioural flexibility is present in the tool skills of free-living NC crows. First, they manufacture three kinds of hook tools out of stick-like material: live twigs, fern stolons, and a thorny vine (Hunt \& Gray 2002). Each kind of tool requires material-specific manufacture techniques for its production. Second, individuals in the same population can specialize in different tool types. At one site on Maré Island individual crows generally specialized in either stick tools or Pandanus tools (Hunt \& Gray 2007). Preferences for a particular tool type were not closely associated with the sexual size dimorphism in NC crows (Kenward et al. 2004). Third, we tested if two crows made tools of an appropriate length in response to holes of different depth (Hunt et al. 2006). On the first attempt in a trial both birds made tools of a consistent length regardless of hole depth. However, they generally made longer tools on the next attempt with deeper holes, which suggested that the crows relied on a heuristic strategy to solve tool problems. Last, the behaviour of a crow that we observed crafting hooked tools out of live twigs was organised in a flexible, hierarchical way (Hunt \& Gray 2004).

A consequence of the diverse range of tool designs that NC crows manufacture is different sets of designs at sites (Hunt \& Gray 2002; 2003). The differences are striking even when the main types of designs are included. NC crows on the island of Maré only make wide Pandanus tools and non-hooked twig tools. Crows at many sites on the mainland make stepped Pandanus tools and hooked twig tools. At certain other sites they make narrow and stepped Pandanus tools and hooked twig tools. In Parc Rivière Bleue, crows surprisingly make wide, narrow, and stepped Pandanus tools as well as hooked twig tools. There are no obvious ecological or genetic correlates associated with these site differences. Therefore, using the "geographic method," which relies on circumstantial evidence (e.g., Whiten et al. 1999), the manufacture of these tool designs appears to meet the requirements for socially learned behaviour.

NC crows are capable of innovative behaviour. We recently documented innovative tool modification in the wild on Maré by two male NC crows (Hunt et al., submitted). The males used a novel folding technique to bend wide Pandanus tools into a boomerang-like shape. A practical consequence of the folding was that it reduced a tool's length without cutting the tool. This allowed the crows to hold longer tools near the working end and in line with their bills. Experiments with captive NC crows have also revealed innovative tool skills that are similar to, or even surpass, those of great apes (Taylor et al. 
2007; Weir \& Kacelnik 2006; Weir et al. 2002). The positive correlations in birds and primates between innovation frequency, tool use, and brain size are also consistent with the evidence indicating that the tool skills of NC crows are learning-based behaviour (Lefebvre et al. 2002; Reader \& Laland 2002).

The final line of evidence is the prolonged development of tool skills in juvenile NC crows. Juveniles forage with their parents in family units for 10 months or more after they fledge and have ample opportunity to learn tool skills in a social context (Hunt \& Gray 2007). It takes many months for them to become proficient in wide Pandanus tool manufacture and use (Holzhaider et al., in preparation). We also have experimental evidence that proficiency in these skills probably depends on appropriate behavioural strategies developed through associative learning (Holzhaider et al., in press).

The authors" belief that "Baldwinization" of behaviour eliminates learning, and therefore the potential for innovation, is based on a flawed understanding of the extent to which learned behaviour becomes genetically assimilated. The assimilation of adaptive learned behaviour is suggested to occur through two different mechanisms: the "Baldwin Effect" described by Baldwin (1896a; 1902) and Waddington's (1942; 1953) "genetic assimilation" (see West-Eberhard [2003] and papers in Weber $\&$ Depew [2003] for detailed discussion about these two mechanisms). "Genetic assimilation" in this commentary refers to both these mechanisms. The importance of the role that genetic assimilation has had in the evolution of animal behaviour remains unknown (Hall 2003). Genetic assimilation of a learned behaviour may also lead to a "learned trait complex" in which supporting adaptive modifications (e.g., morphological and cognitive) refine and elaborate the trait in an ongoing cyclical process as long as it remains adaptive. The important point about this process is that although the adaptive behaviour becomes increasingly specialized and efficient through genetic assimilation, it is still learned behaviour. Genetic assimilation does not eliminate learning, and with learning the potential for innovation is retained (Bateson 2004; West-Eberhard 2003).

Sterelny's (2004) scenario of how technological evolution in humans came about describes a learned trait complex. He suggests that only the basic elements of human technical capability were genetically assimilated (e.g., morphological attributes and cognitive skills). Sterelny stresses that "the total package would remain a gradually elaborating mix of learned and innate elements" ( $p$. 298). Technological evolution would be initiated when individuals used the assimilated attributes and skills in association with learning to innovate and then transmit these innovations. If technological evolution of tools has occurred in NC crows, as we have suggested (Hunt \& Gray 2003), it may have proceeded in a similar manner to Sterelny's scenario for humans (Hunt 2005). This is because only the basic elements of NC crows' technical capability have probably been genetically assimilated.

\section{ACKNOWLEDGMENT}

Many thanks to Felipe Medina for helpful comments.

\section{Objectivism should not be a casualty of innovation's operationalization}

DOI: $10.1017 /$ S0140525X07002440

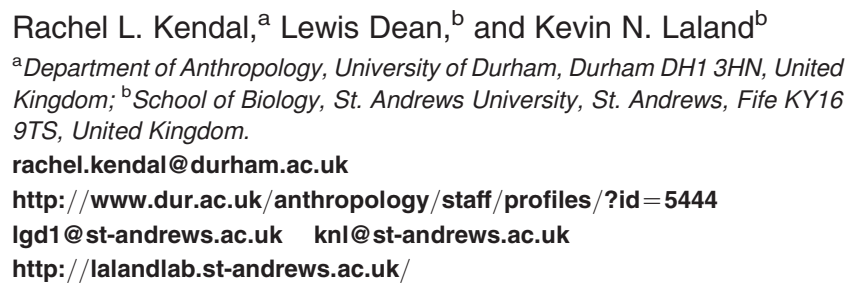

${ }^{a}$ Department of Anthropology, University of Durham, Durham DH1 3HN, United

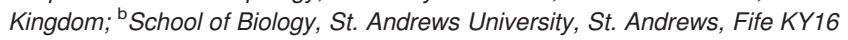
9TS, United Kingdom.

Igd1@st-andrews.ac.uk knl@st-andrews.ac.uk

http://lalandlab.st-andrews.ac.uk/
Abstract: We agree with Ramsey et al. regarding the need for new methods and concepts in the study of innovation, and welcome their initiative, but are concerned that their operationalization is over-reliant on subjective judgements.

The study of animal innovation is in its infancy. Notwithstanding recent interest in the topic, fuelled by the observation that a species' innovativeness correlates positively with brain size (Lefebvre et al. 1997; Reader \& Laland 2002), the only book on this topic was published in 2003 (Reader \& Laland 2003b; cf. Reader \& Laland 2003a), and the number of published articles dedicated to it remains less than 30 . These observations, when juxtaposed against the thousands of articles and tens of books on animal social learning, reveal animal innovation to be a fledgling and understudied domain. We welcome Ramsey et al.'s stimulating article, and enthusiastically endorse their goal of operationalizing animal innovation. Although we express reservations over aspects of their scheme, we applaud their efforts and encourage others to engage in the exercise.

We agree with Ramsey et al. about the need for new methods and concepts in the study of innovation, but begin by maintaining that the Reader and Laland (2003a) definitions are not as problematic as claimed. They were explicitly operational: "The above considerations lead us to the following two operational definitions" (Reader \& Laland, 2003a, p. 14, emphasis in original). The definitions were deliberately broad and encompassing, they eschewed reference to particular psychological processes, and were designed to be practical to implement in both the laboratory and the field. The aspect of Reader and Laland's definitions to which Ramsey et al. object, the requirement that innovations introduce novel behavioural variants into a population's repertoire, is a central part of that operationalization, in that an innovation can be designated when first observed, with no requirement that the original innovator be identified. This has left innovations (sensu product) easy to quantify, and extensive databanks have been collated on innovation frequencies in both primates and birds. True, such numbers are biased in a variety of ways, but researchers have devised statistical procedures to account for these (Lefebvre et al. 2004; Reader \& Laland 2002). Contrary to Ramsey et al.'s suggestion, the approach has facilitated comparisons in innovativeness between species, and allowed the relative rates of innovation in sex, age, and social rank categories to be investigated (Kendal et al. 2005; Lefebvre et al. 1997; Reader \& Laland 2001; 2002). Nor is innovation, by Reader and Laland's definition, necessarily a function of population size: group and population size are not correlated with innovation rate in primates and birds, respectively (Nicolakakis \& Lefebvre 2000; Reader 2006). We do not pretend that Reader and Laland's definitions are perfect, or cannot be improved upon, but nonetheless they exhibit considerable currency.

In contrast, we are concerned that the Ramsey et al. operationalization is overly reliant on subjective judgements of individuals or coworkers. Judgements are required about how frequently or reliably the novel behaviour appears in the population (i.e., enabling exclusion of environmental induction), how rapidly they increase in frequency (i.e., deemed indicative of social/asocial learning), the reliability of "indicators of social learning," the identification of ecological correlates of innovation, and so forth, all of which will vary among researchers. Ramsey et al.'s key is only tenable if each decision could be made reliably and objectively - but could they? There are an infinite number of ecological correlates to a behaviour: how many should a researcher consider before moving to the right in the key? Little consideration is given to the problem of identifying social learning within a population: There is currently no available metric with which to evaluate the decision point "The behavior is known to propagate via social learning" - this is never known with certainty (Laland et al., in press). We fear that criteria will be assessed differently by different investigators, leaving the 
identification of innovations somewhat arbitrary. Of course, there are concerns over the subjectivity of existing innovation databases, but at least the Reader and Laland criteria are clear, objective, and measurable.

Leaving aside its subjectivity, there are other features of Ramsey et al.'s approach that worry us. We disagree that a behaviour pattern should be precluded from categorisation as an innovation because it is seen to be a response to environmental change (see also Reader commentary). Considerable available evidence indicates that much innovation is governed by the adage "necessity is the mother of invention" (Reader \& Laland 2003a). In a search of primate journals, approximately half of recorded innovations, where data were available, were prompted by ecological challenges such as periods of food shortage, dry seasons, or habitat degradation (Reader \& Laland 2001). Similarly, many innovations are induced by the social environment, for example, one in which young/subordinate animals are forced to innovate in order to obtain food or mates monopolised by older/higher-status individuals. This is an awful lot of data to throw away, and Ramsey et al. leave themselves vulnerable to false negatives. Just as social learning is expected to be adaptive and tied to the environment, so is innovation. Ramsey et al.'s claim, that behaviour patterns with strong fitness consequences are unlikely to be innovations, is entirely unproven.

Some aspects of Ramsey et al.'s approach also appear contradictory. We applaud the authors' use of captive animal resources in their method. The presentation of natural or captive populations with novel puzzles or challenges and recording the characteristics of innovators and contextual variables, as recommended by Kummer and Goodall (1985), has proven an effective experimental approach to the study of innovation (e.g., Kendal et al. 2005; Laland \& Reader 1999; Morand-Ferron et al. 2004). However, a logical extension of Ramsey et al.'s line would be to dismiss this experimental approach completely such behaviour patterns are, after all, "environmentally induced," so should we therefore conclude that we are not studying innovation?

The authors acknowledge that their method bears similarity to the ethnographic ("geographic") method, which has been used to infer the presence of cultural traits in primates and cetaceans, but this method is a contentious model on which to base their scheme (Laland \& Janik 2006; Reader commentary). We welcome Ramsey et al.'s emphasis on "rigorous application," but they will acknowledge that the ethnographic method has yet to be applied to culture with sufficient rigor to satisfy all. Thus far, readers have had to content themselves with the opinion of the authors that no ecological correlates were present: they were rarely listed or subject to statistical analyses. This approach to science, in which verdicts are given by a jury of authoritative experts, leaves us uncomfortable. There is much to commend in Ramsey et al.'s approach to innovation, but objectivism must not be a casualty of its operationalization.

\section{Animal innovation and rationality: Distinguishing productivity from efficiency}

\section{DOI: $10.1017 / \mathrm{S} 0140525 X 07002452$}

\section{Elias L. Khalil \\ Department of Economics, Monash University, Clayton, Victoria 3800, Australia. \\ elias.khalil@buseco.monash.edu.au}

Abstract: For the authors of the target article, innovations are underdetermined by environmental inducement - but are still adopted in the future. For a productive technology to be adopted, the technology must also be efficient. To be efficient, it must be determined by constraints or environmental inducements. However, this contradicts the authors' notion of "underdetermination." To make matters clear, we need to distinguish between two kinds of determination: the "source" of the innovation as opposed to "inducement" that makes the organism adopt it in the future.

Ramsey et al. define innovation as "repertoire modification involving the addition of a new behavior, or the modification of an old one, underdetermined by maturation, the environment, and the behavior of conspecifics" (target article, sect. 3.5, para. 6). They also define innovation as modification which lasts into the future and, hence, differs from "improvisation." This commentary shows that Ramsey et al.'s definition of innovation is inconsistent.

Although Ramsey et al. recognize that innovation is influenced by the environment, they insist that innovation is "underdetermined" by the environment. But Ramsey et al. seem to use the term "underdetermination" in two inconsistent senses: "source" versus "inducement" of behavior. First, they use "underdetermination" to mean that the source of the innovation is the organism's voluntary imagination, while recognizing that the environment exercises some influence. Second, Ramsey et al. use the term to differentiate "repertoire flexibility" (innovation) from "behavioral flexibility": The environment induces behavioral flexibility but not repertoire flexibility. Their concept of "inducement" corresponds to the economist's concept of "incentive." The organism, for example, might demand more of fruit A than fruit $\mathrm{B}$ in one year if fruit $\mathrm{A}$ turns out to be sweeter than fruit B. This expresses the law of demand: price of sugar/effort has decreased for fruit A. Ramsey et al. would call such a change of behavior "behavioral flexibility" - where the environment "determines" action in the sense of inducement. For them, there is no such "inducement" determination for repertoire flexibility - because it is already established that it has no "source" determination.

Granted that repertoire flexibility has no "source" environment determination, would it then also have no "inducement" environment determination? This need not be the case if we distinguish "source" from "inducement." This distinction becomes clear from the proposed delineation, in light of rationality theory, between productivity and efficiency (Khalil 2000).

Economists use the concept of "rational choice" to cover both behavioral flexibility and behavioral repertoire. Economists broadly have two definitions of rationality. The "technical" definition of rationality specifies the axioms, such as transitivity and completeness, that guarantee consistent ends (Kreps 1990). The "action" definition specifies that, in response to inducements (i.e., incentives), the organism chooses actions to enhance wellbeing. The action definition presupposes the technical definition, but not vice versa. Gary Becker (1976, Ch. 1), among many others, employs the action definition to study diverse behaviors ranging from the demand for apples, to the demand for spouses, and the sharing of income with loved others (altruism). The action definition predicts, for example, that an agent would demand more of product $\mathrm{Y}$ when its price, ceteris paribus, decreases. It also predicts, for example, how the coal tit bird shops carefully for the eucosmid moth, Ernarmonia conicolana, which inhabits the patches of trees at different densities (Tullock 1971)

The action definition of rationality includes the assessment of whether or not to adopt new productive technologies in the long run and, hence, have them become innovations. Economists recognize that innovation involves a change of technology and, hence, arises from a source that is not determined by environmental changes such as climatic change or sudden discoveries. But when it concerns inducement, that is, efficiency optimization, the source of the change of resources is irrelevant. The source can be environmental change or productivity change.

For a productivity change to be viable, that is, for it to become an innovation, it must meet necessary and sufficient conditions. The necessary condition entails that the productivity change is an improvement in comparison to old technology, that is, it 
expands the resource base or budget constraint. The sufficient condition entails that the productivity change is efficient, that is, it enhances well-being in light of opportunity costs. As the Venn diagrams (Fig. 1) show, if productivity change meets the necessary condition, it is part of the "Productivity Set," whereas if it meets the sufficient condition, it is also part of the "Efficiency Set." Only a subset of the "Productivity Set" can also fulfill the sufficient condition, that is, the "Efficiency Set." This shared subset, here called "Innovation," does not consist of elements ranked in absolute terms according to their productivity alone, that is, along a progressive view of evolution that abstracts from inducement or efficiency - a full cost-effectiveness consideration. In rationality theory (as well as in natural selection theory; Khalil 2007), innovations are ranked in relative terms, that is, according to environmental constraints or inducements.

Note that the "Efficiency Set" is not limited to innovations, or changes in resources arising from productivity. The "Efficiency Set" also includes behavior arising from change of climate, predators, or prey, that is, "Behavioral Flexibility." Also note, the "Productivity Set" is not limited to innovations; it also includes nonviable productivity improvements, that is, "Improvisation." Although improvisations are productive, they are inefficient and, hence, not repeated. So, "Innovations," as proposed here, are novel technologies that exhibit greater productivity and efficiency by the fact that they are adopted in the future.

Ramsey et al. offer a different definition of innovations underpinned by the implicit view that makes the "Efficiency Set" and the "Productivity Set" external to each other. Therefore, Ramsey et al. do not view innovations as "inducement" determined by the environment in the same manner as "behavioral flexibility" - probably because they do not want to sacrifice the thesis, undisputed here, that innovations, related to the issue of "source," are underdetermined by the environment.

In Ramsey et al.'s example of the chimp Eureka and the smashing of nuts, Eureka introduces a new technology when she starts to use stones instead of traditional tree branches. The stone technology would pass the necessary condition, that is, become part of the "Productivity Set," if it entails productivity improvement as measured by output/energy, where energy is measured as if the branches and stones are equally accessible to the organism. That is, the productivity measure does not include environmental accidents such as the relative abundance of the potential tool. The productivity measure only includes energy expended in using and, given capital depreciation, repairing the tool. When we include the issue of relative abundance, we are invoking the sufficient condition in which the opportunity cost of fetching one tool as opposed to another is important. If the organism (or natural selection) judges the stone technology to be more productive than the branch technology, the organism (or natural selection) can still judge the stone technology to be less efficient than the branch technology. In judgment of efficiency, which determines whether the more productive technology is inside or outside the "Efficiency Set," the organism (or natural selection) is induced by the issue of relative abundance. If Eureka spends too much time searching for stones relative to searching for branches, the stone technology would be less efficient, even though it could be more productive, than the branch technology. In this case, the stone technology would not be

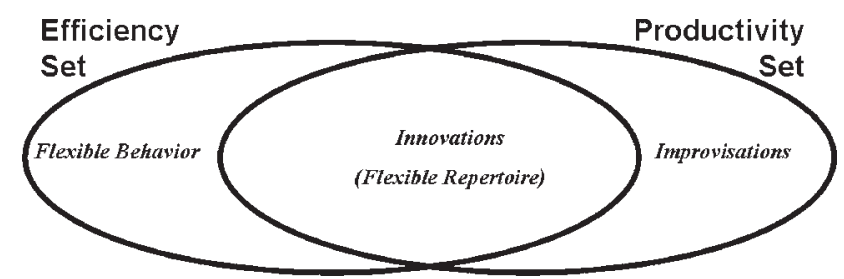

Figure 1 (Khalil). Distinguishing Productivity from Efficiency. repeated, even though it is more productive than branches. If Eureka adopts the stone technology in future actions, it must have passed the sufficient condition, that is, it is part of the "Efficiency Set." Once the stone technology is adopted, it is determined by the environment, in the "inducement" sense, whereas it can be underdetermined by the environment, in the "source sense."

Thus, given Ramsey et al.'s definition of innovation as a modified repertoire repeated in the future, such repetition must entail that the innovation is determined by the environment, at least in the sense of inducement. But in their definition of innovation, Ramsey et al. exclude environmental determination in any sense, including inducement. So, Ramsey et al.'s definition of innovation is inconsistent.

\section{Vocal innovation}

\section{DOI: $10.1017 /$ S0140525X07002464}

\section{John L. Locke}

Department of Speech-Language-Hearing Sciences, Lehman College, City University of New York, Bronx, NY 10468.

john.locke@lehman.cuny.edu

Abstract: An important form of innovation involves use of the voice in a new way, usually to solve some environmental problem. Vocal innovation occurs in humans and other animals, including chimpanzees. The framework outlined in the target article, appropriately modified, may permit new perspectives on the use of others as tools, especially by infants, and the evolution of speech and language.

Although the innovation framework offered by Ramsey et al. is aimed at nonhuman primates, their scheme, or a modification, is needed for humans, especially in connection with vocal innovation. This behavior, fully expressed, involves the use of the voice in a new way, usually to solve some environmental problem. One can witness vocal innovation, and the emergence of social and sensorimotor precursors, in development; and it has been proposed that aspects of this capability played a role in the evolution of speech and language (Locke \& Bogin 2006; Oller 2004).

Whether humans have the capacity to invent new sounds and sound sequences is hardly in question. One only need consider the universal distribution of languages, numbering in the thousands, that are spoken; and their voluminous phonological and lexical systems, which appear to exceed communicative needs. But this says nothing about the development of the control systems required to effect new vocal forms and combinations, nor does it tell us about the evolution of the capacity to produce a range of phonetic elements at will.

In the past, avian researchers were responsible for much of the animal work on vocal innovation. Marler (1991) reported that in songbirds, "recasting or re-editing of components of learned models into new sequences is commonly exploited as one means for generating novelty and also for producing the very large individual repertoires that some birds possess" (p. 61). As described, these cases would evidently meet the definition of innovation set forth by Ramsey et al.

A challenge for linguistic evolutionists inheres in the fact that chimpanzees, unlike the songbirds, are frequently silent, seemingly more inclined to gesture than to vocalize (Tomasello et al. 1985; 1989). But two recent reports indicate that captive chimpanzees issue putatively novel or unusual signals - extended grunts and labial trills ("raspberries") - if they want food or a tool that is in the possession of a human (Hopkins et al. 2007; Russell et al. 2005). This work raises a question for Ramsey et al.: How does their scheme handle behaviors that previously occurred only in an "old" context that, because of some change, came to be emitted in a "new" context? 
Commentary/Ramsey et al.: Animal innovation defined and operationalized

Clues to the functionality of vocal signals are available in other studies, indicating that chimpanzees tend to use them selectively, when the experimenter is looking away (Hostetter et al. 2001; Leavens et al. 2004). It is also of some interest that in chimpanzees, unlike the other primates, there are significantly more preadult, and fewer adult, cases of innovation than would be expected by chance (Reader \& Laland 2001), though it is evidently not known, at present, whether juveniles are also more likely to innovate vocally.

Because new forms emerge in development (West-Eberhard 2003), our attention is drawn to the role of infancy in vocal innovation. An important problem that is solved by infants is the negotiation of their own care during a time when care is likely to be interrupted (Trivers 1974). I have claimed elsewhere that at one or more points in evolutionary history, increased competition for care stimulated novel and more flexible use of vocal behaviors (Locke 2006; Locke \& Bogin 2006).

But signaling involves more than signals. For repertoire change, infants would also need to observe the effect of any new vocalizations on others. Ramsey et al. say little about cases where one individual uses other individuals as a tool. The exclusion of such cases would seem to ignore the social nature of many primate groups, wall off our own exceptionally social species from other primates, and complicate the process of evolutionary theorizing.

Human mothers are disposed to repeat their infants' speechlike vocalizations (Pawlby 1977), a practice that appears to reinforce their use (Veneziano 1988). In fact, some frequently babbled patterns are taken up by family members and may diffuse through the community (Ferguson 1964; Locke 2004; 2006). One is curious to know, in this connection, if Ramsey et al., who exclude cases where individuals witnessed a behavior and its effect on a conspecific, also wish to exclude cases in which an individual's own behavior elicited reactions by others.

Vocal innovation by infants may provide parents with a free evaluation of their offspring's fitness (Locke 2006). In primates and other animals, there are associations between innovativeness, brain development, and learning potential (Lefebvre et al. 2004). It is interesting, in this regard, that human infants who produce a high rate of syllables per utterance appear more pleasant, friendly, and likeable than infants who vocalize less complexly or rhythmically (Bloom \& Lo 1990; Bloom et al. 1993).

It is paradoxical, given the robustness of vocal innovation in humans, that developmental evidence has been reported only sporadically. Observers have noted infants' use of a distinctive intonation pattern when making "requests" (Dore 1974; Ninio et al. 1994), and there is evidence that human infants - like adult chimpanzees - make grunts and labial trills before they begin to speak (Kim et al. 1996; McCune et al. 1996). For some reason, these sounds may be particularly characteristic of retarded infants and children (O’Neill \& Happé 2000; Smith \& Oller 1981). It has also been reported that typically developing infants use a specific class of (nasally emitted) sounds as "requests" (Goldman 2001).

In babbling, human infants effect a variety of closures within the vocal tract while phonating, thereby producing audible activity that, in its most canonical form, resembles consonantvowel syllables and, therefore, speech (Oller 2000). A more advanced form, variegated babbling, involves different points of articulation within a single sequence of syllables. This activity demonstrates, and may facilitate, what Oller (2004) calls "contextual freedom," seemingly a phonetic form of Ramsey et al.'s "behavioral flexibility." Later in development, there is evidence for invented words by young children (Becker 1994; Leopold 1949), who are also responsible for the phonological, lexical, and grammatical inventions that occur in the creation of creoles from pidgins (Bickerton 1984). It is clear, additionally, that adolescents play a major role in linguistic change, particularly at the phonological level of language (Eckert 1999; Labov 2001).
Ramsey et al. have provided us with a framework for the evaluation of innovation across primate species. It would be interesting to see what modifications are required for vocal innovation. Working within a properly designed system - one that values synergies between evolution and development - it may be possible to identify candidate processes in the evolution of speech and language (Locke, in press).

\section{Social learning is central to innovation, in primates and beyond}

\section{DOI: $10.1017 /$ S0140525X07002476}

\section{Corina J. Logan ${ }^{a}$ and John W. Pepper ${ }^{b}$ \\ ${ }^{a}$ Ecosystem Services Section, Washington State Department of Natural Resources, Olympia, WA 98504-7016; ${ }^{\mathrm{b}}$ Department of Ecology and Evolutionary Biology, University of Arizona, Tucson, AZ 85721. itsme@ CorinaLogan.com \\ http://www.CorinaLogan.com jpepper1@email.arizona.edu http://eebweb.arizona.edu/Faculty/Bios/pepper.html}

Abstract: Much of the importance of innovation stems from its capacity to spread via social learning, affecting multiple individuals, thus generating evolutionary and ecological consequences. We advocate a broader taxonomic focus in the field of behavioral innovation, as well as the use of comparative field research, and discuss the unique conservation implications of animal innovations and traditions.

We agree with Ramsey et al. on the importance of an operational definition of innovation. This will clarify communication and direct our focus to significant examples of innovation for closer analysis. However, we suggest a shift in the emphasis. It is useful, as Ramsey et al. do, to discriminate between novel individual innovation and socially learned behavior. However, they focus on the former to the extent that social learning is primarily considered something to discard in the search for examples of "true" (purely individual) innovation. Ramsey et al. define innovation as a process and product attributable to a single individual. To this end, they exclude social learning from the phenomenon they define and discuss. Individual innovations are certainly interesting and worthy of study. However, the importance of innovation stems mostly from its capacity to spread via social learning.

Although ultimately originating from single individuals, innovations become most important when they transcend their solitary origins and become more prevalent. As the authors point out, innovation is a key component of most definitions of culture, and is important because it can affect a species' ecology and evolution. In both respects, innovations are relatively insignificant when restricted to a single individual. An innovation can become part of a culture only through social learning. Likewise, innovations are important factors in the ecology and evolution of a species only when shared by many individuals. Although it may be possible for multiple individuals to independently produce similar innovations, this is not what we typically see in nature. Instead, when innovations become sufficiently widespread to play an essential role in a species' ecology and evolution, they usually have done so through social learning (Laland \& Hoppitt 2003; Laland \& Janik 2006; Mann \& Sargeant 2003; Yurk et al. 2002).

There are important benefits to a broad taxonomic scope in the study of animal innovation. Primates are of special interest to anthropologists because of their relatedness to humans. However, some of the most important questions surrounding the capacity for innovation concern its evolutionary origins and consequences and its ecological effects. The most powerful tool in biology for addressing such questions is the comparative method (Freeman \& Herron 2004; Harvey \& Purvis 1991; Perry 2003). The strength of comparative analysis depends on 
Commentary/Ramsey et al.: Animal innovation defined and operationalized

how many taxa and independent origins are considered for the phenomenon of interest. Primates present many of the bestknown examples. However, animal innovation is taxonomically widespread, and other groups are also noteworthy for the frequency and importance of behavioral innovation. We discuss two examples next. Incorporating as many taxonomic groups as possible in our consideration of innovation can greatly increase the strength and generality of our inferences.

Cetaceans (toothed whales and dolphins) have exceptionally large brains, high levels of intelligence, mental flexibility, and a capacity for behavioral innovation (Marino et al. 2007). Field studies on wild cetaceans have revealed a diversity of behavioral traditions apparently derived from individual innovations (Rendell \& Whitehead 2001). As is true of primates, many behavioral traditions in bottlenose dolphins (Tursiops truncatus) involve specialized foraging techniques (Chilvers \& Corkeron 2001), and some also involve tool use (Mann \& Sargeant 2003). To date, the best information comes from one long-term field study (Mann \& Sargeant 2003). Through comparison across multiple populations, we could better understand which behaviors are instinctive or environmentally induced, and which are valid examples of innovations that have become distinct local traditions. Clearly, there is opportunity for such comparative field study in this species, as several potential examples of behavioral traditions and innovation have not yet been investigated. For example, many unusual foraging specializations have been identified in various populations of bottlenose dolphins in and around the Gulf of California (Leatherwood 1975).

Behavioral innovations are well documented in crows (Corvus spp.) and other corvids (Emery \& Clayton 2004b). Examples include tool use in wild populations, with evidence for social transmission and cumulative social evolution (Hunt \& Gray 2003). It has been proposed that corvids and apes share the same "cognitive toolkit," including abilities for causal reasoning, prospection, imagination, and flexibility (Emery \& Clayton $2004 \mathrm{~b}$ ). The reason that similar cognitive traits have evolved in both groups may be that both needed to solve similar socioecological problems, including locating and exploiting unpredictable food resources, and understanding relationships among individuals in large social groups (Emery \& Clayton 2004b). Similar selective pressures have also been implicated in the evolution of the cognitive abilities of cetaceans (Marino et al. 2007).

Although both have their strengths, comparative field research offers several advantages over captive studies for investigating innovations. Ramsey et al. stressed field studies at the beginning of their article, yet promoted captive studies for confirming innovations. Captive studies can only determine the degree to which a behavior is instinctive, but cannot usually confirm that a particular behavior is an innovation in wild populations. Moreover, the ecological and evolutionary implications of captive studies can be difficult to interpret because of the altered and restricted physical and social environment, especially considering the fact that behavior in large-brained social animals is very sensitive to context (Rendell \& Whitehead 2001). Such species are precisely where Ramsey et al. predict finding more innovation. Laboratory experimentation facilitates isolating behavioral variables, whereas field studies examine these variables in their evolutionary context (Bateson 2003; Perry 2003; Smith et al. 2002; Whiten et al. 2005).

The existence of behavioral innovations maintained through social learning, could have a large impact on conservation efforts by giving distinct conservation significance to each unique population. Complementary to the importance of protecting genetic pools, the preservation of behavioral traditions could add a new dimension to conservation priorities and strategies (Whitehead et al. 2004).

\section{ACKNOWLEDGMENTS}

We thank Christopher Montero for excellent feedback on this commentary.

\section{Innovation in sexual display}

\author{
DOI: 10.1017/S0140525X07002488
}

\section{Joah R. Madden}

Department of Zoology, University of Cambridge, Cambridge, CB2 3EJ, United Kingdom.

jrm54@cam.ac.uk

http://www.zoo.cam.ac.uk/zoostaff/bbe/Madden/Joah1.htm

Abstract: Much attention has been paid to innovative foraging methods, but little to innovative sexual displays. Innovations may be common in behavioural display, such as song or object use, and could occur in both male display form and female preferences. Similar evidence exists for innovation in display as in foraging methods, but in smaller quantities. Ramsey et al.'s methodology permits rigorous data collection in this field.

The new methodology proposed by Ramsey et al. (2007) has implications for studies in areas beyond the ones they highlight. The majority of their examples, and those in the cited literature, are concerned with foraging methods, or coping with novel or changeable environments. It is striking that one well-studied branch of behaviour is underrepresented. Courtship displays, usually exhibited by males, provide a basis for female choice and offer some of the most elaborate behaviours seen or heard in animals (Anderson 1994). Whilst many displays are morphologically fixed (long tails or bright colours) and likely strongly influenced early in life by genetic factors, others are behaviourally flexible, and candidates for innovation. Innovation may occur in the display form (e.g., song structure, movements, use of objects) and in female preferences (mate choice copying, alternative exploitation of a pre-existing bias). Two examples of sexual display in which innovations are possible are bird song (Marler \& Slabbekoorn 2004) and bowerbird's bowers (Frith \& Frith 2004).

Why are innovations in sexual display of interest? First, the variety of elaborate sexual displays still demands an explanation. Innovation, coupled with learning (ten Cate \& Rowe 2007), provides an additional mechanism to traditional genetic mechanisms of inheritance to explain the rapid appearance and divergence of displays. If this leads to mating isolation of certain populations, then it may also inform the understanding of speciation.

Second, sexual display provides an unusual situation where selection favours innovation per se. Females may prefer innovative males, regardless of the exact form of display exhibited, favouring males that contrast with peers (Burley \& Symanski 1998; Elias et al. 2006), perhaps because unusual males are less likely to be relatives, or because innovative males possess a general cognitive ability or can bear a costly brain (Miller 2000). Males exhibiting exaggerated displays, such as large song repertoires, have high mating success (Kroodsma 2004) and one way to extend the repertoire is by generating novel syllables or phrases (Johnson 2006). These become available to other males to copy and incorporate (Slaabekoorn et al. 2003).

Third, the apparent decoupling of reward for innovation (mating or reproductive success) from the display itself provides a useful system to study how individuals decide whether to retain innovations. Sexual displays differ from the exemplar behaviours given by Ramsey et al. (2007). In most cases, such behaviours produce an immediate reward (e.g., food becomes available). A close link between behaviour and reward will likely reinforce the innovative behaviour. Additionally, the reward can be perceived by observers, offering a stimulus for copying (Caldwell $\&$ Whiten 2003). Conversely, rewards for producing an innovative display may be long delayed and concealed from observers. Females can continue to sample males and only subsequently return to a preferred novel male (Uy et al. 2001). Males may be unsure of their reward in terms of reproductive success, especially if they provide no parental care. Feedback following innovation demands further investigation. 
What evidence is there that displays could have originated as innovations? There exist similar types of evidence as for nondisplay behaviours, such as novel foraging techniques, but currently in smaller quantities. First, individuals may spontaneously produce song syllables (Johnson 2006), or incorporate unusual objects into their display (Frith \& Frith 2004), only some of which they retain in subsequent displays. Second, displays may not be genetically fixed, but are refined as the individual ages (Gil et al. 2001; Vellenga 1986). If innovative individuals are copied, then their innovations spread in the population. Finally, populations differ in the displays that males give, independent of environmental or genetic factors, and match patterns of male association (Madden et al. 2004; Marler \& Tamura 1964; Uy \& Borgia 2000).

Innovations in male display may be initiated in response to innovative female choice. If females copy the mate choices of individuals (e.g., Swaddle et al. 2005), and make "errors" in learning - for example, learning to target an incorrect set of cues, or by copying the "wrong" individual - then a new pattern of preferences becomes available for males to exploit using innovative displays (ten Cate \& Rowe 2007). As long as the choice has neutral fitness consequences and the erroneous female is copied by others, runaway processes could rapidly favor the expression of an innovative display coupled with an innovative preference (Laland 1994; but see Kirkpatrick \& Dugatkin 1994). Alternatively, female choice can facilitate innovative displays if her mating decision is based on a pre-existing physiological, neurological, or psychological bias (Endler \& Basolo 1998). If the bias is generalised (e.g., colour), males potentially have numerous ways of stimulating it. For example, a bias for specific colours derived in a foraging context, is stimulated by male morphological colour patches that match that preference (Rodd et al. 2002). Innovation can drive the incorporation of novel colour-matched objects into a male's display (Madden \& Tanner 2003).

Why has little attention been paid to innovation in sexual display? First, innovation in display may be rare, with strong selection against novel displays that are uninformative or noisy. Second, maybe displays are not really innovations. All sexual signals ultimately serve the single purpose of attracting a mate, and so at one level, the behaviour has not changed and no new behaviour has been added to the individual's repertoire. However, by comparison with traditional innovations, there are many ways to crack a nut and these alternative solutions are classed as innovations. In the case of displays, various novel objects could stimulate the same sensory biases exhibited by females, providing scope for innovation in display form, but not its ultimate function. Third, some variation in display form between populations has been explained by environmental (Handford 1981, Madden \& Balmford 2004) or genetic (Baker et al. 1982) factors. Correlations with differences in these factors may be accepted as explanations for the causes of difference, but it is also possible that random innovative change in display could prompt certain males to favour a particular environment in which their display is most conspicuous, or to mate assortatively with certain females, leading to genetic similarities making current genetic differences meaningless when considering origins of innovations. Finally, innovations in display may be very subtle - a novel syllable interjected in a song of many different syllables, or the incorporation of one novel object on a bower already hosting a hundred different object types, providing a daunting task to scientists. The new methodology of Ramsey et al. (2007) addresses this and offers a means of rigorously assessing innovations in sexual display, and so permits expansion of the study of innovations into this novel and potentially fruitful field.

\section{ACKNOWLEDGMENTS}

Thanks go to Katherine McAuliffe and Nikolaus von Englehardt for comments and discussion.

\section{Individual invention versus socio-ecological innovation: Unifying the behavioral and evolutionary sciences}

\author{
DOI: $10.1017 /$ S0140525X0700249X
}

\section{Lauren McCall}

National Evolutionary Synthesis Center, Durham, NC 27705.

lauren.mccall@duke.eduｗww.nescent.org

Abstract: Great promise for the evolutionary analysis of animal behavior lies in the distinction between generative novelties and the evolutionary innovations to which they can give rise. Ramsey et al. succeed in emphasizing the contribution of individual learning and intelligence to behavioral innovations, but do not correct the tendency to confound individual invention with socio-ecological or group-level innovation.

Innovation is a key concept to both the behavioral and the evolutionary sciences for much the same reasons, yet the two fields differ in their definitions. Behavioral scientists do not currently distinguish behavioral "innovation" from "improvisation" or "invention," identifying all of these as a novel behavior's first occurrence. By contrast, an evolutionary innovation is the spread and fixation of a developmental novelty throughout the population or group. Ramsey et al. make a helpful distinction in passing, between behavioral novelties and their cultural entrenchment (sect. 2). Yet they do not examine the dichotomy any further because they are primarily interested in invention, or as they call it, the "individual-level process definition of innovation."

Whereas many researchers of behavior assign innovations to individuals (see Reader \& Laland 2003b), interdisciplinary biologists seek a consensus with the long-standing use of the term in evolutionary biology (Erwin \& Krakauer 2004). The evolutionary meaning of innovation is applicable because behaviors are like other phenotypes, differing only in the larger role the environment often plays in their expression. Learned behavioral novelties and inventions are developmental novelties, which spread by environmental (rather than genetic) mechanisms such as public information (Danchin et al. 2004), social learning, or any other socio-ecological change, whether shared by mass migration or a shift in the environment.

The substantive contribution of Ramsey et al.'s article is its emphasis on the importance of individual learning and intelligence to behavioral innovations, as compared with social learning. It was once assumed that social learning improves fitness by exempting individuals from the costs of individual (asocial) learning. Individual learning carries high costs because trial and error, for example, can have fatal consequences in circumstances such as learning to forage and avoiding poisonous foods, or learning to fly and avoiding falling. However, Rogers (1988) shows that sparing the costs of individual learning would not lead to increased fitness in the population. Like cheaters exploiting a social system based on the expectation of "altruistic" reciprocity, social learners increase their fitness when they are rare, but decrease overall fitness when they are common. Laland and Kendal (2003) describe how social learning continues to depend on individual learning even when conditions are most favorable for learning socially. Behavioral innovations depend on individual learning (e.g., conditioning and other forms of environmental induction) because social learning alone cannot track changes in the environment without individually learned input (Henrich \& McElreath 2003). But are these opposing strategies heritable, or is social learning just individual learning in the context of the social environment? Developmental psychologists working with primates, Fragaszy and Visalberghi (2001) found that the various kinds of learning are not specialized for exploiting particular kinds of information, and that "[t]here is no way to distinguish 'social learning' from 'asocial' learning ... Socially biased learning is distinguished by the context in which learning occurs, not by distinctive cognitive processes" (p. 83). 
If learning is a primary contribution to the generation of novelties that make successful evolutionary innovations, this calls into question the existence of separate vocabularies and explanatory methods and the opportunistic borrowing of concepts among "sibling" natural and behavioral sciences. Like biological mutations, novel behaviors can open the possibility both for their own eventual innovation and for effecting innovation in other traits. Behavior's effects include evolutionary patterns related to the Baldwin effect (1896a) and genetic assimilation (Waddington 1957), which at the limit may be irreversible, either because they constitute a "selective ratchet" preserving functional integration or an "epigenetic ratchet" in which the ancestral state is unregainable on account of feature loss or specialization (Futuyma 1998, p. 693). Behavior can reinforce and even alter evolutionary trends through the building, maintenance, or shift of ecological niche (Odling-Smee et al. 2003). The capacity of the individual organism to affect evolution is a variable that is never at the level of chance, since organisms range from seeking adequacy (e.g., along chemical gradients) to intentionally optimizing their environments through invention. In particular, the choice of mates in sexually reproducing species can become a powerful force through the positive feedback of sexual selection, and have a runaway effect. Finally, behavior may affect evolution in its degree of plasticity or adaptability. Two examples are mobility, which allows for encounters with new possibilities for and conditions on evolutionary change (Lloyd Morgan 1896; see Bateson 1988; 2004), and cognitive plasticity, which can be beneficial particularly in environments that allow trial and error. Contextualizing the study of behavior within the scope of evolutionary biology offers powerful tools in the discussion of innovation.

\section{Context-specific neophilia and its consequences for innovations}

\section{DOI: $10.1017 /$ S0140525X07002506}

\section{Claudia Mettke-Hofmann}

Smithsonian Migratory Bird Center, National Zoological Park, Washington, DC 20008.

Mettkec@googlemail.com

http://www.orn.mpg.de/mitarbeiter/mettke.html

Abstract: According to Ramsey and colleagues the main constituent psychological processes of innovation are response to novelty, exploration, and the ability to recognize a novel solution. I fully support this view but point out that novelty reactions are often context-specific. I will expand on this and discuss the possible consequences of contextspecific novelty reactions on the emergence of innovations.

Intuitively, one may expect that the reaction to novelty is a general property of an individual applicable across a wide range of situations - an individual that readily investigates a change in the environment, such as a newly emerging food source, may also hesitate little to investigate an unfamiliar environment beyond its territory. However, reactions to novelty are often context specific. For example, individual Pumpkinseed-sunfish (Lepomis gibbosus) that more rapidly approached a meter-stick were not necessarily the first to investigate a novel food source (Coleman \& Wilson 1998). In Carib grackles (Quiscalus lugubris), levels of neophilia (attraction to novelty) were correlated within different contexts (investigating unfamiliar objects, tasting novel food, or entering unfamiliar space), but not across contexts (Reader 2003). In genera as different as parrots and songbirds, neophilia differs across closely related species in a context-dependent manner depending on the ecology and migratory strategy of a species. For example, when compared to migratory species, year-round resident species explore changes in their familiar environment more rapidly and thoroughly (Mettke-Hofmann 2007; Mettke-Hofmann et al. 2005a; 2005b), whereas migratory rather than resident species are more likely to explore a novel room (Mettke-Hofmann et al., under review). These differences in neophilia have likely evolved as an adaptation to the migratory strategy of the species (Mettke-Hofmann et al. 2005b). Likewise, still-invading populations of house sparrows (Passer domesticus) feed from a novel food source more quickly than long-established populations, even though the two populations showed similar levels of neophobia (avoidance of novelty) when a novel object was presented next to familiar food (Martin \& Fitzgerald 2005).

Given that novelty reactions (a) differ intraspecifically across contexts, (b) are interspecifically differently expressed across contexts depending on the ecology and migratory strategy of a species, and (c) are the main underlying mechanisms of innovations, it is likely that innovations emerge predominantly in contexts in which an individual or species shows the most neophilia. This raises the following question. Are neophilic reactions in all contexts equally likely to favor innovations, or are neophilic reactions in one context more likely to lead to innovations than those in other contexts (contextual bias)? Findings from Reader (2003) in the Carib grackle seem to favor the latter. The number of different attempts to solve a foraging task (a measure of innovation) was most closely associated with object neophilia (approaching an unfamiliar object) than with neophilia to taste a novel food or enter a novel room. However, the foraging task involved manipulating a Plexiglas box to reach food (Webster \& Lefebvre 2001). What if the foraging task had involved a spatial context, such as finding a way into a familiar room with food (e.g., trying several entrances)? Would there have been an association with spatial neophilia?

The fact that many of the innovations described in the literature include manipulating items, tool use, or novel food suggests that object and food neophilia may be more prone to induce innovations than spatial neophilia. This may also reflect a bias for detecting innovations towards the former context, however. Object- or food-related innovations are probably easier to identify than spatial innovations such as use of a different strata or movement technique, or the use of a novel habitat. In birds, only $13 \%$ of the reported innovations (augmented version of the database used in Lefebvre et al. 2004) can be classified as spatial innovations, although birds may be more likely than other taxa to show spatial innovations because of their threedimensional use of the environment. In Bornean Orangutans (Pongo pygmaeus wurmbii; van Schaik et al. 2006), 5\% of the 43 innovations reported classify as spatial innovations.

To solve the problem of contextual versus detection bias we need studies that (1) pay special attention to spatial innovations and (2) investigate the relationship between neophilia and innovations within and between contexts across a broad range of species. Resident-migrant comparisons are ideally suited to address this problem as residents show more object neophilia and migrants more spatial neophilia (Mettke-Hofmann 2007; Mettke-Hofmann et al. 2005a; 2005b). Resident palaearctic species are also more innovative than migratory counterparts (Sol et al. 2005b). However, it is currently unknown whether residents have a higher innovation rate than migrants due to a contextual bias for innovations to emerge preferentially in object-related contexts, or whether the former just have more object-related innovations, whereas the latter may innovate equally often or even more often in spatial contexts, although we miss it because of a detection bias.

In addition to object-related versus spatial innovations, such comparisons may also include object-related versus foodrelated innovations. Distinguishing between object-related and food-related innovations is important, as shown in the study of the Carib grackle (Reader 2003) where object neophilia but not food neophilia was mostly associated with the foraging task. Similarly, differences in food neophobia but not object 
neophobia distinguished expanding and established house sparrow populations (Martin \& Fitzgerald 2005).

Investigating the relationship between novelty reactions and innovations contributes to our understanding of the underlying mechanisms of innovations and allows us to predict the context in which innovations are most likely to occur. To accurately compare innovativeness across species, as suggested by Ramsey et al., we not only have to take into consideration differences in social transmission, but also the fact that biases may exist towards detecting innovations more frequently in certain contexts. Awareness of the context-specifity of innovations further helps to make comparisons more reliable. Consequently, interspecific comparisons should control for the context in which innovations occur by restricting comparisons to object-related or food-related innovations until other forms of contextdependent innovation have been systematically measured.

\section{ACKNOWLEDGMENTS}

Thanks to Louis Lefebvre and Niels Rattenborg for helpful comments and to Louis Lefebvre for providing original databases.

\section{Environmentally invoked innovation and cognition}

\section{DOI: $10.1017 /$ S0140525X07002518}

\section{Simon M. Reader}

Behavioural Biology, Department of Biology and Helmholtz Institute, Utrecht University, Utrecht 3508 TB, The Netherlands

s.m.reader@uu.nl http://www.bio.uu.nl/behaviour/Reader/

\begin{abstract}
Behavioral innovations induced by the social or physical environment are likely to be of great functional and evolutionary importance, and thus warrant serious attention. Innovation provides a process by which animals can adjust to changed environments. Despite this apparent adaptive advantage, it is not known whether innovative propensities are adaptive specializations. Furthermore, the varied psychological processes underlying innovation remain poorly understood.
\end{abstract}

Ramsey et al. argue that behavioral innovation should play an important role in studies of animal social learning, evolution, ecology, and cognition. I agree entirely, and admire their work in establishing and moving the field forward. As there remains much to do, it is important, particularly at these early stages, to reach consensus on clear definitions and delimitation of phenomena. Ramsey et al. argue for revision of Reader and Laland's (2003a) definition of animal innovation, principally excluding environmentally induced novel behavior and defining innovation at the individual rather than population level. Both revisions are debatable. Although a group member can innovate even if another has made the same discovery, such innovations are difficult to discriminate from socially learned innovations, whereas first occurrences may be particularly relevant to identification of the individual and/or environmental variables that favor innovation. Ramsey et al.'s individual-level definition also raises problems regarding the distinction between innovation and individual learning (see Reader \& Laland 2003a).

Ramsey et al. exclude environmentally induced innovations from consideration, but change in the physical or social environment may precede much innovation (Hauser 1988; Lee 1991; Reader \& Laland 2001), and innovative bird species are more likely to survive than less innovative species when introduced to novel environments (Sol 2003). Moreover, many (but by no means all) reported innovations are responses to humaninduced environmental changes (Lefebvre et al. 1997; 2001; Reader \& Laland 2002). Innovation may also be prompted by social demands, where it may be key in outwitting rivals (e.g., innovative tactical deception; Byrne 2003), be frequency dependent, or be prompted or facilitated by existing innovations that create a need or opportunity for further innovation. All these would be considered environmentally induced innovations, but they are innovations nonetheless, and surely of both theoretical and applied interest (e.g., to conservation biology; McDougall et al. 2006). The exclusion of environmentally induced innovations, as Ramsey et al. advocate, will likely eliminate false positives, but may also exclude many interesting and functionally important "true" innovations. Innovation presumably carries maximal advantage as a response to novel circumstances where natural selection cannot have shaped appropriate responses.

Ramsey et al.'s new definition and classification scheme may carry other disadvantages for empirical studies of innovation (Kendal et al. raise complementary points in their commentary). Ramsey et al. propose several characters of potential utility for discriminating innovations from non-innovations, and present a useful exposition of how these characters can be considered together. However, the rate of acquisition, rate of spread, rarity, apparent cognitive complexity, and distribution within or across populations may provide misleading clues for identifying innovations. Such characters are not irrelevant to the study of proposed innovations, but should be interpreted cautiously: many genetic, social, environmental, and experiential influences can impact on the performance of a given behavior. Proper consideration of ecological explanations for behavioral differences between populations, for example, requires consideration of the availability, utility, and risks of alternative behavior patterns. The relative utility and performance of a given foraging behavior could be influenced by, for instance, the availability of other foods, the ease of identifying alternate foods, differences in need (e.g., disease promoting peculiar nutritional requirements), local predation risk, the activities of others (e.g., resource competition), or constraints on performance of alternative behaviors (Dewar 2004; Reader 2004). Exclusion of such "ecological" causes for population differences may be extremely challenging with observational study alone (Laland \& Janik 2006). Moreover, as argued earlier, ecological causes would not eliminate the possibility that the behavior was an innovation.

Ramsey et al. suggest using geographic and local prevalence to identify innovations, but how informative are these variables? Innovation prompted by the peculiarities of local sub-habitats would result in a patchy distribution that would correlate with ecological differences. Conversely, with limited solutions to the same problem, an innovative species may repeatedly and independently invent the same behavior in many populations, resulting in high geographic prevalence. Nocturnal feeding by street lights in kingbirds, for example, has been repeatedly reported on several Caribbean islands, suggesting environmental induction in each population (Reader et al. 2002a). Innovative species may also show high local prevalence of novel behaviors. As Ramsey et al. note, reported novel behavior is amenable to experimental investigation in the laboratory, in the field, and with wild-caught captives (e.g., Morand-Ferron et al. 2004; Pfeffer et al. 2002; Reader et al. 2002b), and such experiments provide a vital follow-up to observational data. Experimental investigations of celebrated cases of animal innovation - sweet-potato washing by macaques and milk-bottle opening by titmice - demonstrate that individual rates of discovery may be far higher than was generally thought (Kothbauer-Hellman 1990; Sherry \& Galef 1984; 1990; Visalberghi \& Fragaszy 1990). Where geographically or locally prevalent innovation suggests that a novel behavior is readily discovered, species comparisons may be informative: Why do some species apparently make these discoveries readily, while sympatric species do not? High local and geographic prevalence in particular species could result from several processes, including general behavioral flexibility, context-specific flexibility, or Ramsey et al.'s "Baldwinized innovations." However, although Baldwin effects have significant theoretical impact for the evolutionary consequences of behavioral flexibility (Sol 2003; Wyles et al. 1983), they lack clear empirical support (de Jong \& Crozier 2003). 
Here are two final definitional points. First, Ramsey et al. introduce "behavioral flexibility" to mean behavior-level flexibility. However, current usage typically considers behavioral flexibility as part of phenotypic plasticity and thus repertoire, not behavior-level, flexibility (Bateson 1983; Schlichting \& Pigliucci 1998). Second, the suggested social learning-innovation continuum is unclear and open to several alternative interpretations: (1) Some individuals innovate while others socially learn these innovations; (2) some elements of a behavior are innovated and some are socially learned; (3) social learning involves some innovation, such as when stimulus enhancement attracts a bird to food but the processing methods are individually acquired; or (4) copying errors during social learning result in novel behavior.

An important question not raised by Ramsey et al. is whether innovative propensities are adaptations rather than side-products or indicators of other phenomena, such as general behavioral flexibility. Investigation of the genetic, hormonal, neurocognitive, and developmental mechanisms underlying innovation will help resolve this issue as well as the important questions raised by Ramsey et al. and Reader and Laland (2003a). There is clear potential for integration with research on temperament and behavioral syndromes (Réale et al. 2007; Sih et al. 2004). Although some evidence suggests that innovative propensities may be consistent individual traits (Laland \& Reader 1999; Pfeffer et al. 2002, but see Fragaszy \& Visalberghi 1990), it is not known whether innovatory propensities are heritable, are impacted by developmental experience, or whether animals can "learn to innovate." A range of psychological processes may be involved in any one innovation, and may differ between different innovations. Progress will require investigation of the causes, consequences, and mechanisms underlying animal innovation; all areas ripe for study.

\section{ACKNOWLEDGMENTS}

I am grateful to Neeltje Boogert, Rachel Kendal, Matthew Bruce, and Ulf Toelch for helpful comments, Kevin Laland for discussion, and the Netherlands Organisation for Scientific Research (NWO) Evolution and Behaviour Programme for funding.

\section{Is all learning innovation?}

\section{DOI: $10.1017 /$ S0140525X0700252X}

\section{Luke Rendell, ${ }^{a}$ William Hoppitt, ${ }^{\mathrm{a}}$ and Jeremy Kendal ${ }^{\mathrm{b}}$ \\ ${ }^{a}$ School of Biology, University of St. Andrews, St. Andrews, Fife KY16 9TS, United Kingdom; ${ }^{\mathrm{b}}$ Department of Anthropology, Durham University, Durham DH1 3HN, United Kingdom. \\ ler4@st-andrews.ac.uk http://bio.st-andrews.ac.uk/staff/ler4.htm wjeh1@st-andrews.ac.uk http://bio.st-andrews.ac.uk/staff/wjeh1.htm jeremykendal@gmail.com \\ http://www.dur.ac.uk/jeremy.kendal/index.html}

Abstract: Research on animal innovation is an underdeveloped field, and for this reason we welcome the efforts Ramsey and colleagues have made to stimulate its study in wild populations. However, we feel that in attempting to find an operational definition the authors have overstretched the idea of what we should consider innovation in some areas and over-restricted it in others.

Although we welcome new thought aimed at stimulating research on animal innovation, we find problems with the definitional approach Ramsey et al. have taken, especially with defining innovation at the level of the individual. We are unconvinced by the restriction on cases involving what the authors term "environmental induction" (proposing instead a distinction between "passive" and "active" innovation), and on cases with significant positive fitness impacts. Finally, we don't believe the distinction between behaviour and repertoire flexibility is useful.
First, we do not believe that defining an innovation as something new to the individual, rather than the population, makes sense. No doubt it is individuals that innovate, and it is possible that more than one individual in a population might produce the same innovation independently. However, if innovation is simply another term for asocial (or individual) learning, we have unnecessary redundancy. The concept of innovation at the population level is useful, because it pertains to the process by which new behaviours enter into populations, with potential evolutionary impacts. At the individual level, the concept becomes entangled with processes such as trial-and-error learning that we might describe under the broad term "asocial learning." Furthermore, some forms of learning require both social and asocial processes. For example, individuals may be attracted to a new food source through stimulus enhancement, but then use trialand-error learning to acquire the extraction method. Therefore, perversely, "innovating" as defined by Ramsey et al. would be subsumed within a form of social learning. The commitment to innovation at the individual level is also at odds with our societal sense of what an innovation is - we have a patent system precisely to establish the primacy of whoever first came up with something new. Can all individual learning be innovation? We think not.

One possible response to this question is to restrict innovation to those cases not resulting from environmental induction. This separation is unconvincing, not least because most examples of innovation are linked to novel opportunities in the environment. The adaptive value of innovation, indeed behavioural flexibility in general, is surely in dealing with environmental variation. Thus, periods of environmental change are precisely when one might expect higher levels of innovation. Perhaps a landslide caused a pile of stones to appear next to a tree suddenly - does this mean their subsequent use cannot, by definition, be an innovation? Milk-bottle opening by blue tits in the United Kingdom is widely touted as an innovation, but without the environmental induction of soft-topped milk bottles, would never have occurred.

Nonetheless, understanding the role of environmental change in innovation is potentially worthwhile. However, we would draw a rather different distinction to that made by Ramsey et al. Their hypothetical example of chimpanzees switching to stone tools after a forest fire illustrates the point. The switch to stone tools could only occur through a phase of operant learning by the chimpanzees: the use of stones can only be reinforced once an individual has experimented, and succeeded in using one to crack open a nut. Thus, an environmental change may act to provide either the opportunity or necessity for operant learning, which, in the case of the first individual to succeed, we would term "innovation." We feel such cases are fundamentally different to cases in which an environmental event directly alters the behaviour of an individual. An individual may learn through being passively exposed to the relationship between two stimuli in its environment. For instance, after being attacked, an individual may learn to associate a particular scent with the arrival of a predator. In future, it may respond to that scent with fear or escape responses. If the individual is the first in the population to form this association, it might be said to be the innovator. We would term that individual a "passive innovator." In contrast, an individual who has learned to use a stone hammer, through operant learning, we would term an "active innovator." We feel this distinction is likely to prove useful as active innovation is more likely to reflect the cognitive abilities of the innovator than passive innovation, which is more likely to rely on chance events.

These issues aside, the target article gives the impression that it would be easy to distinguish non-innovation based on environmental induction, but this is far from the case. For example, the claim that environmentally induced novel behaviours are expected to be adopted rapidly by most of the population following a change in the environment does not hold water if the rapid 
spread was a result of (1) asocial learning, if, for example, the task is easy to solve (i.e., "innovation" sensu Ramsey et al.) or (2) horizontal social transmission of the innovation, which can also be rapid.

The assertion that behaviours with large positive fitness impacts should not be considered innovations because they are likely to have been "Baldwinized" to become at least partly innate, is also unconvincing. We think a stance that discards putative cases of innovation a priori because of the possibility of this having occurred is overly restrictive. The relative contribution of genetic and learned sources of information in the development of behaviour is an empirical question, and there are several lines of possible evidence available to researchers to address it. When this is taken alongside the "environmental induction" restriction, we arrive at a slightly bizarre situation in which we are asked to not consider putative cases of innovation that result in large fitness payoffs in the face of environmental change, when it is precisely these cases that are likely to favour innovation.

Finally, we were confused by the distinction made between behaviour and repertoire flexibility. For example, the authors state that stone hammering is a repertoire addition, but we could argue that actually it is just flexibility in a general "hammering" program: hammer with whatever seems to be a good tool at the time. Similarly, it is unclear why elements of behavioural flexibility cannot be learned, for example, if stone use were subsequently reinforced and resulted in operant learning, and therefore innovations? Deciding whether a particular case pertains to the behavioural or repertoire level will not be an easy task; and in the absence of evidence that the brains of a particular species actually function in this way, it seems to us rather a futile one.

In summary then, we do not believe the case for redefining innovation to the individual level is made, nor is it made for the restrictions on environmental induction or high fitness impacts this redefinition necessitates.

\section{Innovation and the grain problem}

\section{DOI: $10.1017 / \mathrm{S} 0140525 X 07002531$}

\section{Anne Russon, ${ }^{a}$ Kristin Andrews, ${ }^{b}$ and Brian Huss ${ }^{c}$ \\ a Department of Psychology, Glendon College, York University, Toronto, Ontario M4N 3M6, Canada; ${ }^{\mathrm{b}}$ Department of Philosophy, York University, Toronto, Ontario M3J 1P3, Canada; ${ }^{\mathrm{C}}$ Department of Philosophy, State University of New York-Potsdam, Potsdam, NY 13676. \\ ARusson@gl.yorku.ca andrewsk@yorku.ca hussbd@potsdam.edu http://www.yorku.ca/andrewsk}

Abstract: Our concern is with Ramsey et al.'s method for identifying innovation. We show that either it yields false positives or the authors offer insufficient guidance for its application. To avoid these results, the authors need to modify the key or offer better guidelines for delineating input. Either choice requires addressing the processes that generate a behavior.

Ramsey et al. have made a valiant effort to identify innovations in nature. As their theoretical perspective on innovation as a product largely conforms to Reader and Laland (2003a), their novel contribution is epistemological. They may well have considered as much information as possible on the ecological, individual, and historical factors that suggest innovations in nature. However, their method does not incorporate all the factors discussed in the text. For this reason, we believe that Ramsey et al. face a dilemma. If their key takes any behavior as input, many behaviors that are not innovations will be identified as such. Alternatively, if it takes only behaviors that count as potential innovations, then the grain problem looms large. We conclude that the solution requires information on the innovation process.
To demonstrate, consider an unusual orangutan behavior seen in nature: leaf carry (LC), the collection of nest materials before reaching the nest site instead of afterward (Russon et al. 2006). Prevalence patterns suggest LC may be a candidate for innovation: it is patchily distributed, rare at Tuanan (wild), and cultural at Kaja (rehabilitants). At Tuanan, only three orangutans LC using leaves from a single species; the key identifies Tuanan LC as probably an innovation, which is potentially correct (van Schaik et al. 2006). At Kaja, LC probably originated in ex-captives during cage life, where leaves for bedding must be collected before going to a nest site. That is, it was ecologically induced elsewhere and introduced to Kaja by immigrants, so by Ramsey et al.'s standards it is not an innovation. LC did not transfer immediately from cage to Kaja life; it lay dormant in some orangutans for over 18 months. We tried the key for Kaja LC. Q1 asks whether long-term field data are available, that is, longer than the behavior's mean latency. Available data included 7 months of observation at Kaja, earlier records for Kaja and prerelease, and background knowledge on rehabilitation and captive living. LC latency is unknown, so the conservative answer to Q1 is "F." Q1(F) leads to Q19(T), Q26(F), Q40(T), Q41(F), Q43 (probably an innovation), which is probably incorrect. If we accept these data as long-term, the key sequence is Q1 $(\mathrm{T}), \mathrm{Q} 2(\mathrm{~F}$, cage learned), Q14(T), Q15 (probably an innovation) or Q1(T), Q2(T, first Kaja occurrence observed), Q3(T), Q4(T), Q5 (probably an innovation). Ramsey et al.'s example of Eureka's stone tool use meets the same fate if Eureka returns to her original community, introduces stone tool use, and stone tool use subsequently attains cultural status. This is not an innovation by Ramsey et al.'s standards, but of the five plausible routes through the key, all but one identify it as probably an innovation; further, only one of these paths suggests that considering behavior in other populations would strengthen the conclusion. We found similar problems with other behaviors.

In addition to highlighting several points about innovation (e.g., cultural behaviors introduced by immigrants may not represent innovations; dispersal, migration, and dormancy seriously confound the behavior's origins), these cases suggest that if any behavior can be entered into the key, false positives will be common. The only way to deal with this horn of the dilemma is to take process into account, because it is the knowledge of generative processes which suggests these key decisions are false positives.

The other horn of the dilemma raises two problems with specifying inputs to the key: how we determine that behavior is novel and how we delineate behaviors. Both issues concern grain - the level(s) at which an actor acquires and organizes its behavior. Grain reflects the steps an actor takes in acquiring behavior, hence the shape and pace of innovation. Ramsey et al. raise the grain issue but develop it only briefly.

Since few if any behaviors are entirely novel, delineating inputs entails determining what components of a behavior are new to the actor (Byrne 2003). Not only does this require evidence of what the actor already knows, it also depends on the steps the actor uses in acquiring behavior. To delineate behaviors, Ramsey et al. suggest assessing whether behaviors are functionally different. Functional differences should reflect the actor's point of view, so again we need to know how actors delineate their own behaviors. It is then not possible to determine what behavior to enter into the key without knowing what grain the actor uses.

In our view, resolving the grain issue is critical because grain can vary between and (developmentally) within taxa. To illustrate, consider the great apes. Mature great apes seem to organize their behavior at multiple levels that have been characterized as action elements (behavioral detail, e.g., target items, motor actions), procedures (regularly used combinations of components that achieve a specific goal, e.g., using a wiper to clean something), and programs (combinations of procedures and action elements organized to achieve larger goals, e.g., stone nut cracking) (Byrne et al. 2001a; 2001b). Currently, proposed innovations for wild orangutans are inconsistent for grain 
(van Schaik et al. 2006). Leaf napkin for wiping latex off chin, for example, delineates wiper tools by behavioral detail (material leaf, target - chin), but erotic tool does not and even ignores tool type. Evidence suggests that great apes probably innovate at the procedure level, programs are probably socially acquired, and action elements are ecologically induced (Byrne et al. 2001a; 2001b). Programs are probably acquired piecemeal, because great apes' low cognitive ceilings and slow cognitive development suggest that advances are made by small, hard-gained steps (Russon 2003). Taken together, this suggests that both erotic tool and leaf napkin may be inappropriate entries for the innovation key because of errors of grain - the former is too grossly defined (potentially underestimating the number of innovations), the latter too finely (potentially overestimating).

Ramsey et al. think that focus on the products of innovation will help shape the research program on the cognitive factors underlying innovation. Given the potential for false positives and the difficulties associated with the grain problem, we suggest that the relationship is reversed. Only once we understand the cognitive faculties of a taxon and the processes that generate a behavior can we begin to determine whether that behavior is an innovation.

\section{ACKNOWLEDGMENT}

Thanks to Laura Adams for helpful discussion.

\section{Defining and detecting innovation: Are cognitive and developmental mechanisms important?}

DOI: $10.1017 /$ S0140525X07002543

Brooke L. Sargeant ${ }^{\mathrm{a}}$ and Janet Mann ${ }^{\mathrm{b}}$

${ }^{a}$ Department of Biological Sciences, Florida International University, Miami, FL 33199; ' Departments of Psychology and Biology, Georgetown University, Washington, DC 20057.

sargeant@fiu.edu http://www.brookesargeant.com mannj2@georgetown.edu

http://bioserver.georgetown.edu/faculty/Mann/janet.html

Abstract: Although the authors' ingenuity in identifying criteria for innovation for field studies is appealing, most field studies will lack adequate data. Additionally, their definition does not clearly distinguish innovation from individual learning and is vague about cognitive mechanisms involved. We suggest that developmental data are essential to identifying the causes and consequences of learning new behaviors.

Defining innovation is key to studying it; Ramsey et al.'s definition is based on: (1) what processes generate innovation, (2) whether innovations refer to behaviors new to an individual or to a population, and (3) a dichotomous key designed to guide field research.

1. Processes. Ramsey et al. need to clarify what makes innovation qualitatively different from novel learned behaviors that emerge in response to intrinsic (maturational) and extrinsic events. Is the goal to identify specific cognitive mechanisms in order to distinguish innovation from other novel learned behaviors? Higher-level cognition is implicated in most innovation research, but is this a necessary requirement? Ramsey et al. do distinguish between "cognitively simple innovations" produced by accident or trial and error and "cognitively complex innovations" produced by causal reasoning. Although specific learning mechanisms (e.g., trialand-error, directed learning, goal-oriented behavior, or "insight") have been intentionally barred from some definitions of innovation (e.g., Reader \& Laland 2003a) and requiring them creates challenges for field studies, mechanistic criteria can help clarify whether innovation is a subset of or equivalent to individual learning. This is analogous to the debate on whether any form of social learning constitutes culture (e.g., Rendell \& Whitehead 2001). Does any form of individual learning qualify as innovation?

If, to identify innovation, one must exclude maturational and ecological causes as much as possible, the authors' approach suffers from the same shortcomings of the geographic method for identifying "culture" (e.g., Laland \& Janik 2006; Sargeant et al. 2007). Not only would it be difficult to collect sufficient data to exclude ecological or maturational causation, these mechanisms interact to provide adaptive contexts for development of new behaviors and are thus critical to any study of innovation. Ramsey et al. acknowledge that these causes are not mutually exclusive and even suggest that novelty and "food scarcity, risk, and perhaps mobility (leading to 'novelty' when one returns to a location after an absence)" (sect. 5.2, para. 2) are factors underlying psychological processes of innovation, even though these seem to qualify as "environmentally induced." Their approach differs from theoretical approaches that consider rates of environmental change essential factors in understanding selective pressures and adaptive benefits of various types of learning (e.g., Boyd \& Richerson 1985; Laland \& Kendal 2003; Whitehead 2007).

2. Individual versus population. The authors also define innovation as development of non-socially learned behavior novel to an individual, rather than a population. If the goal is to understand cognitive abilities, this point is vital because individual learning is more relevant to cognition than what occurs at the population level. For example, a population of one species exposed to anthropogenic change might exhibit more new, learned behaviors than a pristine population of a closely related species with equivalent learning ability. However, if one accepts this definition, innovation is, again, largely indistinguishable from the broader concept of individual (or non-social) learning. All learned behaviors are, by definition, novel to an individual. Alternatively, studies that define innovation as behavior novel to a population (e.g., Reader \& Laland 2003a) may aid in estimating (albeit conservatively) the rate of individual learning in wild populations (e.g., Reader \& Laland 2002) and also help identify conditions that favor niche expansion and facilitate documentation of the transmission of new and potentially cultural behaviors (e.g., Reader \& Laland 2003b). Thus, we consider "innovation" better defined as a subset of individual learning evidenced by behaviors new to a population, useful for studying conditions favoring the development, spread, or effects of new behaviors.

3. Dichotomous key. We applied Ramsey et al.'s operational definition to our long-term study of foraging behavior in wild bottlenose dolphins (Tursiops sp.) in Shark Bay, Australia. Bottlenose dolphins have obvious "innovative" abilities in that they readily produce novel behaviors in captive (Herman 2002) and field settings in response to human activity (e.g., Chilvers \& Corkeron 2001; Mann \& Kemps 2003). We have identified at least 12 distinct foraging behaviors and substantial individual specialization in our population (Mann \& Sargeant 2003; Sargeant et al. 2005). In applying the key to our data, several challenges were apparent. First, most foraging behaviors were correlated with ecological variation (Sargeant et al. 2005; 2007) and maturation (Mann \& Sargeant 2003). Second, even in a well-studied population, relevant data were lacking. For example, to separate innovations from behaviors that were "environmentally induced" detailed knowledge of the environment at fine spatial and temporal scales is needed, something extremely difficult in studying dolphins that seek mobile patchy, highly variable, aquatic prey. Also, information on whether behaviors are innate or genetically determined or occur in captivity was not available, especially because captive dolphins are fed dead fish. Similarly, determining whether social learning was involved is controversial because standards of evidence and methodology are still hotly debated.

Finally, data from multiple populations might help, but are severely limited by diverse methodologies, observation 
conditions, and research effort. In using the dichotomous key, we had to disregard the clear social and ecological contributions for most foraging behaviors (Mann \& Sargeant 2003; Sargeant et al. 2007) and assume no exclusively genetic causes (e.g., Krützen et al. 2005). Six foraging behaviors fit the criteria for innovation and several were inconclusive. Using Reader \& Laland's (2003a) definition, two behaviors would be classified as innovations: sponging and trevally hunting. Trevally hunting involves an elaborate pursuit and processing of golden trevally fish (up to $1 \mathrm{~m}$ in length), and is used by one adult female (among hundreds of dolphins observed in the population). She catches trevally every few hours, and attracts many dolphins immediately following a catch, suggesting the availability of trevally to others. Sponging would also qualify largely because a small subset of individuals uses this foraging method and we have traced its development and habitat use (Mann \& Sargeant 2003; Sargeant et al. 2007). As expected, Reader and Laland's criteria were more conservative by identifying only relatively recent innovations of known origin.

The authors present an ambitious method for identifying novel learned behaviors that lays the groundwork for a standardized approach, but relevant data will likely be missing for many species. Although the authors attempt to identify ways to study potential innovations using more rapidly obtained cross-sectional data, their approach requires more effort in several areas (more sites, experimental or laboratory data) and it may still take substantial observation to determine which individuals exhibit a behavior. Thus, it is unlikely to "save time" relative to longitudinal study. In addition, their approach does not clarify what cognitive mechanisms are involved. Detailed developmental study on the emergence of behaviors might provide a better indication of maturational, parental, social, ecological, and other influences because these can be directly measured. Sample sizes are a limitation, but detailed developmental analyses can reveal processes involved in the emergence of novel behavior for the individual (e.g., Lonsdorf et al. 2004; Thornton \& McAuliffe 2006). As with culture, developmental data are sorely needed to help identify intrinsic and extrinsic factors that favor novel behaviors.

\section{The animal variations: When mechanisms matter in accounting for function}

DOI: 10.1017/S0140525X07002555

Hugo Viciana ${ }^{\mathrm{a}}$ and Nicolas Claidiere
${ }^{\mathrm{b}}$
${ }^{\mathrm{a} G r u p o ~ d e ~ E v o l u c i o n ~ y ~ C o g n i c i o n ~ H u m a n a, ~ U n i v e r s i d a d ~ d e ~ l a s ~ I s l a s ~ B a l e a r e s, ~}$
Palma, 07012, Islas Baleares, Spain; ${ }^{\mathrm{b}}$ Institut Jean Nicod, Pavillon Jardin,
École Normale Superieure, 75005 Paris, France.
Hugo.Viciana@ens.fr http://www.evocog.com
Nicolas.Claidiere@normalesup.org http://www.institutnicod.org/

Abstract: We contend that Ramsey et al.'s definition of animal innovation sensu process may be partially misleading when investigating mechanisms underlying animal innovation. By excluding social learning from the "process" of innovation, they may be reproducing a dichotomous schema that does not accurately correspond to our knowledge of the acquisition of novel behavioral variants. This gives us some reason to doubt the functional specification of the defined process of innovation.

In their article, Ramsey et al. operationalize animal behavioral innovation in a way that is both an improvement and an extension of current methods for studying innovations. As an attempt to explicitly define the process of innovation at an individual level, as opposed to the population level, their definition could also foster progress in the study of the actual mechanisms of innovation. However, the authors remain agnostic concerning the mechanisms of behavioral innovation. We believe that in the end, this agnosticism may have some undesired side effects.
Indeed, when advancing their descriptive definition of innovation, Ramsey et al. distinguish between the learning of a new behavior by an individual (what they call the "process" of innovation) and the transmission of the new behavior between conspecifics (the spread of the innovation "as a product"). This dichotomous vision of innovation and transmission reflects the distinction between, on the one hand, mechanisms generating new variants and, on the other hand, mechanisms of faithful transmission of these different variants. We fear that this view may be unhelpful when trying to individuate processes of innovation (a purported goal of their definition) from other processes such as social learning.

Let us take, for example, the behavior of British tits (Parus spp.) opening milk bottles. As classical studies have shown, the spread of this behavior was not a matter of general imitation nor a process of exactly copying the behavior of other conspecifics (Hinde \& Fisher 1972; Sherry \& Galef 1984). Rather, those birds that were initially thought to be imitative, partially stimulated by conspecifics, had to re-learn the steps leading up to drinking milk from a previously closed bottle. However broadly construed, social learning, in this case, did not automatically lead bluetits to open milk bottles. Instead, each bluetit had to re-discover on its own how to reach the goal. By characterizing the classic example this way, we wish to convey the idea that the mechanisms that preserve the innovative behavior (sensu product) in the population may sometimes be as innovative (sensu process) as the process by which the first individual produced the first instance of the behavior in that population. We think that this is an important point that could be extended to other paradigmatic cases of diffusion of animal innovations (such as sweet potato washing or termite fishing (Galef 1992). The more general claim is that in the absence of a strong functional justification of the unity of the process of innovation we may expect it to be realized by very different mechanisms across taxa. These mechanisms may not fit with Ramsey et al.'s definition. Similarly, some innovations (sensu product) may often be the result of an accumulation of small modifications produced during relatively faithful transmission between individuals. Consider the case of song learning in certain species of birds: Young birds use the template of a given song sung by their surrounding conspecifics to produce what is, strictly speaking, a new song but one that nevertheless resembles those already existing in the population. This does not count as an innovation according to the definition proposed by Ramsey et al. because that slightly novel variant is functionally determined by its conspecifics and therefore would be classified as a case of general "social learning." Over time, however, the successive transformations accomplished by several individuals may change this given song in the population and therefore produce a completely new and innovative song relative to the first population template (see Slater \& Lachlan [2003] for a review). Indeed, if one bird were to suddenly produce that highly different song, it would, indeed, be classified as a full innovation or "invention" from the perspective of Ramsey et al. But in our example, the process of innovation is distributed so as to make it almost undetectable from the perspective of their definition. If we are right, the understanding of the mechanisms that preserve innovations may be crucial to explaining the creation as much as the diffusion of those same innovations (see Podos et al. 2004). Innovations may thus arise as the outcome of an interindividual process that could not be explained without paying explicit attention to the different mechanisms of social learning. This may well be the default case in humans, a paradigmatic case of an innovative species. In our species, even when the goal of a given behavior is precisely to perfectly reproduce the model, this process is characterized by the systematic introduction of novel idiosyncratic variants that could eventually be the basis of well-known Chinese-whispers-like phenomena (Barbrook et al. 1998; Gergely et al. 2002; Sperber 2001). 
Thus, we think that keeping with a dichotomous view of "innovative processes" and "preservative" or "social learning" processes may be misleading and hinder the characterization of mechanisms at the basis of much of animal innovation. Ramsey et al., on the contrary, insist on the fact that innovation - or "invention," which they define as a paradigmatic case of innovation - does not require either environmental induction or social learning. Thus their claim is that "the individual, process-based definition of innovation excludes social learning as a source of innovation" (target article, sect. 3.3, para. 3; emphasis added). We believe that defining innovations in opposition to social learning in a broad sense, excludes innovations that occur as the result of individuals' interactions in processes other than mysterious "Eureka!" events. Our view is that innovations are not the simple result of a well-defined excluding class of processes. Rather they are the complex outcome of different mechanisms some of which undergo different functional pressures and sometimes concern several individuals. Since the phenomenon of animal innovation cuts across different ecological domains (as varied as song learning or foraging), as well as different animal taxa, expectations of a unified process under the auspices of a general evolutionary function may not be justified (Burghardt 2006). On the whole, we expect Ramsey et al.'s contribution to have enormously positive outcomes. The operationalization that they propose may foster more accurate estimations of the repertoire of innovations of different species. This in turn may facilitate functional investigations into innovation rates - something tremendously useful in itself, but which will also attract further interest in underlying mechanisms.

ACKNOWLEDGMENTS

We thank Coralie Chevalier, John Hulsey, Olivier Morin, and Sabita Claidiere for useful remarks on earlier versions of this commentary.

\section{Authors’ Response}

\section{On the concept of animal innovation and the challenge of studying innovation in the wild}

\author{
DOI: $10.1017 /$ S0140525X07002567
}

Grant Ramsey, ${ }^{\mathrm{a}}$ Meredith L. Bastian, ${ }^{\mathrm{b}}$ and Carel van Schaik ${ }^{\mathrm{c}}$

${ }^{a}$ Department of Philosophy, University of Notre Dame, Notre Dame, IN 46556-

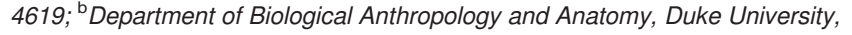
Durham, NC 27708-0383; ${ }^{\mathrm{C}}$ Anthropological Institute and Museum, University of Zürich, 8057 Zürich, Switzerland.

grant.ramsey@nd.edu

http://philosophy.nd.edu/people/all/profiles/ramsey-grant/index.shtml mlb22@duke.edu

http://fds.duke.edu/db/aas/BAA/grad/mlb22

vschaik@aim.unizh.ch

http://www.aim.unizh.ch/Members/vanschaik.html

Abstract: The commentaries have both drawn out the implications of, and challenged, our definition and operationalization of innovation. In this response, we reply to these concerns, discuss the differences between our operationalization and the preexisting operationalization if innovation, and make suggestions for the advancement of the challenging and exciting field of animal innovation.

The target article is an attempt to answer two questions. First, what is animal innovation? And second, can we study innovation in the wild without observing the process of innovation? In answering the first question, we proposed a novel way of defining innovation based on individual-level processes. Several of the commentaries challenged the nature of this definition and the reasons why we constructed it as we did. Because of this, we spend considerable space here clarifying our definition and defending it against the concerns raised in the commentaries. In answering the second question, we have suggested evidence that may increase the probability that a behavior is an innovation. The two main concerns commentators had with this operationalization are whether it would produce false positives/negatives and whether the criteria it uses are objective. After discussing our definition and its operationalization, we turn to some broader conceptual issues, such as the nature of the Baldwin effect and the relationship between this effect and innovation.

Before we begin, we would like to thank the 31 commentators (representing ten countries) for the time and effort they put into carefully reading and reflecting on our target article. We regret that we lack the space to respond to all of the important issues they raised, but hope that we have done justice to their most important points.

\section{R1. On the definition of innovation}

We begin our response with a treatment of the more general critiques of the logic and structure of our definition of innovation.

\section{R1.1. Is all learning innovation?}

Some of the commentators (e.g., Rendell, Hoppitt, \& Kendal [Rendell et al.], Sargeant \& Mann) have suggested that our definition of innovation might be so broad that all learning (or all individual learning) ends up falling under the rubric of innovation. We agree that this would be a problem if it were the case, but fortunately it is not. First of all, our definition explicitly excludes social learning from the process of innovation. By doing this we are not denying the possibility that social learning can prompt innovation or that innovations can spread via social learning. Instead, we are denying the identification of the process of social learning with the process of innovation. We are also not denying the importance of social learning. Pace Logan \& Pepper, we take social learning to be central to the study of innovation, since social learning allows innovations to have effects beyond the individual (indeed, this is the reason we decided to examine the concept of innovation). Viciana \& Claidiere's point that the processes of social learning and innovation can be intertwined is well taken. But the close connection between social learning and innovation does not make them the same process.

A further restriction in our definition is that innovation must be learned but not environmentally induced. As we discussed in the target article, the concept of environmental induction is a tricky one and is revisited in section R1.4. The point we would like to make here is that the restrictions we have placed on the concept of innovation, that it is behavior that is novel, learned (both in the sense of not innate and in the sense of modifying 
Response/Ramsey et al.: Animal innovation defined and operationalized

the organism's behavioral dispositions), not socially learned, and not environmentally induced, clearly mark innovative behavior as a subset of learned behavior.

\section{R1.2. Is our definition tautological?}

Giraldeau, Lefebvre, \& Morand-Ferron (Giraldeau et al.) accuse us of "tautological thinking." In order to understand their critique, let us first say a few words about the nature of scientific definitions and the reasons why we think it is important to develop a new definition of innovation. One role of definitions in science is to refer to new concepts or things. Generally, this is accomplished by introducing a novel term and stipulating its meaning. Such stipulative definitions are neither true nor false, and, although they may be useful, they are not very interesting. Our definition of innovation is not of this kind. Another common kind of definition is (merely) descriptive - the sort of definitions one finds in a dictionary. These definitions - which can be true or false - do little more than help one learn the meanings of terms. The kind of definition of innovation that we have produced is explicative; instead of merely stipulating or describing the use of the term innovation, we have attempted to explicate the concept of innovation, which heretofore has either been only nebulously defined or has been merely defined in operational terms. We feel that in constructing this definition we have been sensitive to the class of phenomena researchers have been picking out as innovations and not deciding "a priori on the characteristics that it should possess if it were to be recognized," as Giraldeau et al. phrased it.

Are we guilty of tautological thinking? A tautology, of course, is a sentence that is true by virtue of its logical form (and is thus independent of matters of fact). Giraldeau et al. are not explicit in identifying such sentences. Instead they say that our "categories appear entirely tautological and far from being amenable to strong empirical study." This statement is a bit puzzling since categories are not the sort of thing capable of being tautological, but we feel that what they mean is that the criteria we propose in our operationalization have been produced a priori, but are in fact based on a posteriori facts. For example, the key in the target article's Figure 2 is based on the putative fact that the near simultaneous appearance of a behavior in a population makes it improbable that it arose through innovation followed by social learning. This inference - from simultaneity of origin to the improbability of innovation - is not based on a priori assumptions about the rate of the spread of innovations. To use a fanciful example, if wild chimpanzees started using cell phones, the pattern of spread of innovations would be different than we would expect otherwise - innovations could appear within and between multiple, geographically isolated populations nearly simultaneously. Such changes in the world would necessitate changes in the key. Giraldeau et al. argue that, "The assumptions used by Ramsey et al. are perhaps correct, but it is far from self-evident and must be the object of scientific scrutiny." We could not agree more. It is an empirical question whether the key is in fact successful in identifying innovations - as we have defined them - in the wild, and it is not immune to modification in light of empirical discoveries.

\section{R1.3. Individual versus population level definitions}

We defined innovation at the individual level, arguing that the process of innovation is an individual-level process. We recognized that this individual-level process would be difficult to observe, and this is why we used the operationalization section in the target article to connect these individual-level processes to more easily observable products and properties.

Sargeant \& Mann suggest that defining innovations at the individual-level may be problematic. The concluding sentence in their Individual versus population section is: "Thus, we consider 'innovation' better defined as a subset of individual learning evidenced by behaviors new to a population, useful for studying conditions favoring the development, spread, or effects of new behaviors." They thus agree with us that it is an individual-level process involving learning, but we disagree on the evidence that counts for marking the behavior as an innovation. They hold that novelty at the population level is the only such evidence. We hold that novelty at the population level is evidence of innovation, but that it is not the only such evidence. We consider other evidence, such as the distribution of the behavior within and between populations, to bear on the question of whether the behavior arose as an innovation.

Kendal, Dean, \& Laland (Kendal et al.) also suggest that the individual-level definition of innovation is problematic and defend the Reader and Laland (2003a) population-level novelty definition. The two main reasons that they argue for the population-level definition is that it makes empirical work on innovation easier and that it is more objective. We answer the question of objectivity in section R2.2. But what we would like to point out here is that we are not denying that population-level novelty is a sign of innovation. We are critical of their approach because (among other reasons) we hold that it rules out the possibility of multiple individuals in the same population producing the same innovation - thus innovativeness will be underestimated. Also, despite the fact that Kendal et al. hold that the population-level novelty definition makes the identification of innovations easier, we feel that it is in fact more restrictive than our approach. Our operationalization does not require long-term studies (like those needed in order to say whether a behavior is truly novel for a population); estimates of innovations can be obtained from cross-sectional field studies with data on the distribution and/or properties of the behaviors.

Some of the commentators that argue against the individual-level definition end up implicitly endorsing it. Consider this quote from Rendell et al., for example: "First, we do not believe that defining an innovation as something new to the individual, rather than the population, makes sense. No doubt ... it is possible that more than one individual in a population might produce the same innovation independently" (our emphasis). Rendall et al. do not seem to see the inconsistency in these statements. If innovation is defined as population-level novelty, then it follows from this that if an individual produces some innovation, $I$, 
then later independent productions of $I$ by other members of the population will not count as innovation. For the latter to be possible, innovation must be defined at the individual level. In fact, it is considerations such as the fact that "more than one individual in a population might produce the same innovation independently" that motivated us to produce an individual-level definition.

A population-level definition of innovation conflates multiple, distinct processes. For example, Deleporte points out the importance of cultural loss in relation to innovation. If one were measuring innovativeness using a population-level definition, the innovation rate (and thus innovativeness) would be a function of the rate of cultural loss, because if a novelty is lost (from the population) and then created anew, the new creation (being novel at the population level) will be recorded as an innovation. On the other hand, if the novelty did not disappear through cultural loss, then independent creations of the behavior would not count as innovation. This seems to us highly problematic: Innovation and cultural loss are distinct processes that should not be conflated. Our individual-level definition does not possess such problems. An innovation is an innovation whether or not the same behavior exists in the population.

\section{R1.4. Environmental induction versus innovative response to environmental novelty}

The most common misunderstandings of our definition of innovation center around the distinction between environmental induction and innovative responses to environmental novelty. Many commentators have taken all environmental involvement in the production of novel learned behavior to be instances of environmental induction. For example, Reader cites the case of kingbirds feeding by street lights in several Caribbean islands and suggests that under the target article's classification system this would be considered environmental induction (and not innovation). But we would suggest that the mere fact that the behavior has arisen independently in multiple locations does not imply that it is environmentally induced. If all kingbirds in the presence of streetlights immediately begin to feed near them, this suggests environmental induction. But if the process is slower, if only one or a few kingbirds exhibit the behavior at first, then many of them begin to exhibit it - this suggests innovation. It is thus not inconsistent with our concept of innovation for the same behavior to arise multiple times independently. In fact, as discussed in the previous section, the possibility of multiple origins of the same behavior is one of the benefits of our individual-level definition of innovation.

Rendell et al. similarly argue that our category of environmentally induced novel behaviors should actually be considered innovations. They say that "Milk-bottle opening by blue tits in the United Kingdom is widely touted as an innovation, but without the environmental induction of soft-topped milk bottles, would never have occurred." Is opening the milk bottles by blue tits an example of environmental induction? This seems doubtful, since this is not a behavior that arose throughout the United Kingdom shortly after the introduction of the foil-topped milk bottles. Instead, the behavior appears to have arisen by innovation in a limited number of cases and then spread.
Rendell et al. introduce the distinction between "passive" and "active" innovation as an alternative to our environmental induction-innovation distinction. We think their distinction is interesting, but do not see it as incompatible with ours. Instead of having "passive" innovation replace our concept of environmental induction, we see it as being intermediate between innovation and environmental induction, perhaps occupying part of the space that we marked off as "weak innovation." We believe at least some of their disagreement with us stems from a misunderstanding of the distinction we draw between innovation and environmental induction, evidenced by the following passage. "We are unconvinced by the restriction on cases involving what the authors term 'environmental induction' [...], and on cases with significant positive fitness impacts." We did not suggest either that environmentally induced behaviors cannot have positive fitness impacts or that innovations must have a positive fitness impact. In fact, our concepts of innovation and environmental induction are independent of fitness considerations. (This is not to say that such considerations cannot come into play when trying to assess whether a behavior is an innovation - see sect. 4.2.2 in the target article for a discussion of how the fitness impact of a behavior can serve as evidence for whether it is an innovation.)

Khalil's conclusion that our definition of innovation is inconsistent also stems from the mistaken idea that innovations must be fitness enhancing. His argument is that in order for innovations to boost fitness, there must be an inducement from the environment. But, he concludes, if innovations are defined in terms of a lack of environmental induction, then the definition is inconsistent. It is quite clear that if innovations are not defined in terms of fitness enhancement - like the fitness-neutral definition in the target article - then his argument does not apply.

The more liberal definition of innovation advocated by our critics is in reasonable compliance with their operationalization, which often includes responses to human-induced environmental changes. We have raised the bar, and asked for more than mere flexibility or the ability to adjust to human-made changes in the environment. Some work suggests that curiosity and problem-solving abilities are not correlated across species (Mettke-Hoffmann), suggesting they are regulated by different factors. To mention just one obvious difference, exploratory tendencies may depend strongly on perceived risks (Gajdon) or on context (Mettke-Hoffman), which may be irrelevant, at least at the proximate level, to problem-solving abilities. In the end, this is an interesting empirical issue, and the answers may even vary by taxon. But we must note that most bird work to date has taken the liberal definition, which probably underlies some of the comments. As we point out later, there is a risk that our critics' approach has measured only this ability to adjust, for example, to human observers or to human-made objects and structures, and not the narrower category of what we have termed innovation, which is more then mere behavioral flexibility.

Gardner discusses the interesting case of cross-fostering. A human-raised chimpanzee, for example, will exhibit a host of novel behaviors. Are all such novelties cases of environmental induction? No, just because the captive individual is exhibiting a behavior not represented 
Response/Ramsey et al.: Animal innovation defined and operationalized

by wild individuals does not mean that they are environmentally induced. It could be the case that captivity provides more opportunities for innovation, or disposes individuals to innovate (see, e.g., Gajdon's kea example), and that many of the behaviors exhibited in such cases are innovations. Comparative studies can help resolve this issue. Comparative studies with cross-fostered individuals can also help us decide whether a behavior observed in the wild is an innovation. If cross-fostered individuals exhibit a behavior also exhibited by wild individuals, this decreases the probability that this behavior is an innovation.

\section{R2. The operationalization of innovation}

The process of innovation is an individual-level process. Because of this, it will be difficult to observe in nature. In the target article, we suggested a procedure for sorting innovative behaviors from non-innovative behaviors without having to observe this process. Several of the reviewers had questions or concerns about this procedure and we will address these here.

\section{R2.1. False positives and negatives}

The operationalization we developed is deliberately conservative, and thus better at avoiding false positives than false negatives. Several commentators argue that we cannot properly deal with the role of ecology. Giraldeau et al. complain that we need to know a lot about the ecology of the species we study before we can decide whether a behavior is an innovation. Similarly, Sargeant \& Mann point out that most foraging behaviors were correlated with ecological variation and maturation. We agree that our approach is likely to produce false negatives, because wherever there is a clear ecological correlate to the presence-absence pattern of a behavior across populations we conservatively assume that the more parsimonious alternative, that of a reliable interaction between innate predispositions and environmental releasers, explains the behavior's geographic variation. There are several ways of dealing with this problem. Perhaps the most practical one is to see if there is extensive peering or other indications that social learning may take place before a maturing individual adopts the behavior. Field experiments furnish another option, provided one can exclude neophobia as an explanation of negative results.

We suggested that comparisons with captivity, especially experimental attempts to elicit the behavior with captive animals by offering them the essential conditions, could be an important additional criterion for deciding whether a behavior is an innovation. Gajdon correctly points out that captive animals may be much less neophobic, and thus more exploratory, and hence more likely to find the solution. Ongoing work with orangutans (by S. Lehner, for example) points in a similar direction. That means that comparisons with captivity may falsely suggest that behaviors of wild animals are not innovations, thus increasing the number of false negatives.

Some commentators raised concerns about the key. Giraldeau et al. ask what we mean by a behavior being common. As pointed out earlier, there are no absolute criteria, and it is largely up to the researcher, so long as it remains consistent between populations or studies. Novelty at the population level is a statistical quantity. Our approach makes the criterion explicit and quantifiable, rather than relying on the intuitive guesswork of anonymous authors. Russon, Andrews, \& Huss's (Russon et al.'s) discussion of leaf carrying in orangutans (the tendency to carry leafy twigs of a particular species around before selecting a site to build a nest) concludes that it is probably not an innovation. We are puzzled by this conclusion, since it is absent from numerous orangutan populations and it is difficult to design a solid ecological explanation for its absence at these sites given its presence at some others. That makes it a strong candidate for innovation. The key is not meant to deal with ex-captive animals that may be ecologically naïve, or had already acquired some part of the repertoire of their population before being moved into captivity, and are then released into a natural habitat that may or may not be the same as the one they came from. The key certainly does not deal with ecological induction in captivity and might therefore misfire when applied to released rehabilitants.

\section{R2.2. Is our operationalization objective?}

Kendal et al. charge that our way of identifying innovations is subjective, or at least less objective than that of Reader and Laland (2003a). They do not define what they mean by "objectivism," but let us assume that they mean something like inter-subjective agreement - that if multiple researchers were told to do the same task, they would produce the same results. Or if different results were obtained, the difference would be due to errors of measurement, not different judgments of the researchers. A proper response to the charge made by Kendal et al., then, requires discovering two things. First, what is the task? And second, to what degree is this task objective?

One task would be to search for words like unusual, novel, or original in the animal behavior literature and then compare the numbers of occurrences of these terms with morphometric data such as brain size. We will refer to this as the anecdote compilation method. (This is the sort of task that Giraldeau et al. promote in their commentary.) Is this method objective? The compilation of the anecdotes may be an objective task, but it is far from clear that the anecdotes were produced with a standardized, objective method. If the anecdotes were not produced in a standardized, objective way, then the claims made by studies using the anecdote compilation approach are undermined. In our target article, we refrained from criticizing this approach. Now that we are criticized as lacking in objectivity, it is perhaps useful to review the differences between the two approaches.

We recognized two major ways of documenting innovations: (1) by recording the first instance in a population subject to long-term study (as per Reader \& Laland's 2003a definition), and (2) by recording properties of the behaviors, such as their rareness within a population or across all populations studied, the behavior's limitation to only some populations but not others, as well as other factors such as the relationship between the behavior and various environmental and social elements. We promoted the second approach as a practical complement to the first one, one that would not require long-term data and that might allow us to identify many more innovations 
than the first, provided we have data on multiple populations.

In assessing the objectivity of our method as well as the anecdote-compilation method, we should enquire into the $\operatorname{method}(\mathrm{s})$ by which the anecdotes were generated. While many anecdotes seem to use approach (1) - evidenced by the use of terms such as novel, unusual, first recorded instance, not noted, and so on - many of them were also likely to have relied on approach (2), for example, a behavior only seen in one place, not in others. It is unlikely that most reports were based on thorough long-term studies of single populations. Indeed, what looks novel or unusual to a mobile observer, visiting numerous sites, may simply be different from the average over multiple populations they have composed in their mind. When terms such as "not reported" or "previously unknown" are used, they are not generally meant to refer to individual populations, but to the knowledge of the species as a whole. Indeed, in many sites where these behaviors were observed, they may have been around for a long time. Inevitably, this means that a mix of approaches (1) and (2) probably underlies the majority of the anecdotes. To put it more bluntly, when commentators criticize our approach, they are criticizing the source of their own anecdotes (the source being "a jury of authoritative experts" [Kendall et al.]). Surely, all should therefore agree that finding a method to replace ad hoc anecdotes with more systematic data, as we tried to develop in our target article, is a priority if we are to make progress. If this reduces the number of acceptable innovations to a trickle (the complaint of Sargeant \& Mann), then so be it: It would be very reassuring to know whether the small, but cleanedup data set obtained this way still produces similar results.

When one relies on the opinions of others, one closes one's eyes to the risk that some of what the authors of the original papers identified as innovations may not be innovations if we use more explicit criteria. It is therefore important to ask whether the anecdote-compilation approach may lead to the inclusion of behaviors that should not be considered innovations or introduce biases in the innovations that get recorded this way. If we examine the list of examples in some of the papers (Lefebvre et al. 1997; 1998; 2004; see also Reader \& Macdonald 2003), we find many instances where all would agree that innovations are the most plausible interpretations, but also quite a few where this is not the case. Such cases may not be innovations in spite of the fact that they are "strange" or "interesting" or "worthy of attention." First, many cases of unusual food items may simply indicate that the animals have broad diets (such as the many examples of consumption of vertebrate prey by animals that usually eat invertebrates, or of fruit by animals that mostly eat animal prey), or that the opportunity to eat some foods simply rarely arises, such as the meat of a stranded whale. Thus, the more we study a particular species, the more we might see these putative examples of innovation on a regular basis (seasonally or on a longer time scale, but predictably shown by the same individuals). In the worst case, then, all we have is an indication of the overall behavioral diversity of a species. Second, for others where some element of novelty or unusualness is clear, some of our other commentators (e.g., Russon et al.) would rightly question whether we deal with true novelty from the perspective of the behavioral decisions made by the animals and as intended by the definition in the target article. To give just a few examples: When house sparrows systematically search car radiator grilles for (squashed) insects, how different is this (to a house sparrow!) from searching any other rough substrate with crevices? Or, when birds follow tractors that flush prey items, how different is this from merely being attracted to moving prey (Lefebvre et al. 1997)? In most of these cases, the answer is not obvious, and detailed case studies, such as those done by Morand-Ferron et al. (2004), are needed to assess the probability that the behavior is an innovation. Until these are done, many behaviors on the list require judgment calls similar to the ones we had to make in the case of applying the technique proposed in the target article (see van Schaik et al. 2006), with the difference that we made them explicitly and suggested further work that could resolve their status. Similar concerns can be raised for the comparative work that uses primate anecdotes.

Even if all the anecdotes used in the comparative analyses are true innovations, one may still ask whether they represent an unbiased sample across species. As Healy and Rowe (2007, p. 458) pointed out, "these accounts might be biased by the visibility of the species, the species' distribution in relation to human populations, and also the energetic needs of the species. Indeed, some of the innovations given as examples suggest hunger as a driving force (e.g., the first report of a rook eating frozen human vomit: Lefebvre et al. 1997)." Visibility to humans may be linked to size or to adaptability to human observation, which may well have neuroanatomical correlates. Mettke-Hofmann suggests that object and food-based innovations may be more likely to be recorded than spatial innovations: "Object- or food-related innovations are probably easier to identify than spatial innovations such as use of a different strata or movement technique, or the use of a novel habitat. In birds, only $13 \%$ of the reported innovations (augmented version of the database used by Lefebvre et al. 2004) can be classified as special innovations, although birds may be more likely than other taxa to show spatial innovations because of their three-dimensional use of the environment." As we saw earlier, there is also a danger that the anecdotes merely capture omnivory or generalism, and it is not clear a priori that such feeding strategies are a direct result of accumulating innovations. During the analysis, further biases may have crept in, such as when the number of records for taxa is corrected for research effort by counting the number of publications on them. That number may be affected by factors such as geographic distribution, use in biomedical or other research, or, indeed, flexibility and thus tractability as research subjects.

These two problems suggest that the results of the anecdote-compilation are subject to bias of unknown magnitude, which may or may not be serious. More worryingly, there is the risk that they have merely shown us the brain correlates of a trait other than innovativeness. Still, the results of the comparative studies have been quite impressive and robust (Lefebvre et al. 2004; Reader \& Laland 2002; Sol et al. 2005a; Timmermans et al. 2000), suggesting clear correlations between brain size and the measures extracted through anecdote compilation. But it does not follow from the link between brain size and the 
Response/Ramsey et al.: Animal innovation defined and operationalized

anecdote compilations that the trait of innovativeness correlates with brain size. The anecdotes, instead of picking out the trait of innovativeness, may instead often be picking out a different trait, such as generalism. Although it would be an interesting discovery to find a positive correlation between brain size and generalism, this is quite distinct from finding such a correlation with innovativeness in the more specific sense used here.

Our comments are not meant to disparage the important comparative work, which we find very exciting. However, as we noted earlier, there are uncertainties, suggesting to some that the results are not credible (e.g., Healy \& Rowe 2007). Moreover, the anecdote compilation work relies on operationalizations of innovation that are implicitly the same as those we proposed in our target article. It is therefore all the more important that a more rigorous independent alternative is developed to complement this approach. The method proposed here is likewise subject to possible biases, but because these are different ones, we feel it is worth pursuing. If independent approaches produce comparable results, we can have more confidence in the correlates of innovation. The operationalization we propose, if successful, will also help to generate novel insights into the conditions that bring out innovative tendencies, and thus allow us to produce hypotheses on the conditions that favor the evolution of innovative abilities.

\section{R2.3. The grain problem}

In the target article section entitled "Delineating behaviors" (sect. 5.1), we pointed out some of the challenges of distinguishing different tokens of the same behavioral type from behaviors that represent genuinely distinct behavioral types. This challenge - the "grain problem" - was discussed by several of the commentators. Rendell et al., for example, use our example of hammering: Are hammering with a stone tool and hammering with a wooden tool distinct behavioral types, or it is merely flexibility of a single behavioral type ("hammer with whatever seems to be a good tool at the time")? Rendell et al. are rather pessimistic about the possibility of making this distinction and hold that one would need (difficult to obtain) knowledge about the mechanisms involved. We are a bit more optimistic and feel that although detailed knowledge about the animal's cognitive mechanisms would be invaluable, purely behavioral observations and experiments will also help make these delineations.

Locke raises the interesting issue of "behaviors that previously occurred only in an 'old' context that, because of some change, came to be emitted in a 'new' context[.]" Would such a behavior be counted as a new behavior or not? Our answer would be that since behavioral novelty is context dependent, it is possible that the behavior is a genuinely new innovation. It just depends on whether the individual is indiscriminately performing the behavior (in which case it would not be considered a new innovation) or whether its performance in the new context precipitated a change in the individual's repertoire (in which case it would be considered a new innovation).

As Russon et al. emphasize, it is not "possible to determine what behavior to enter into the key without knowing what grain the actor uses." We agree that one needs to create a typology of behaviors before one can use the key. But we would like to point out that this is not a problem unique to us. It is a problem for any operationalization of innovation. Reader and Laland's (2003a) population approach fares no better than ours: To claim that a behavior is novel at the population level is to claim that it represents a novel behavioral type (and not mere flexibility of a preexisting behavioral type).

\section{R3. Broader conceptual issues}

In addition to the discussion of our definition and operationalization of innovation, a number of the commentators invoke broader issues surrounding the nature and evolution of innovation. We discuss some of these issues here.

\section{R3.1. Mechanisms and innovation}

We intentionally did not attempt to specify the kinds of mechanisms that lead to innovation. We did this not because we think such mechanisms are unimportant. On the contrary, we think the study of the mechanisms that underpin innovative behavior is vitally important. We simply feel that such research is at too early a stage to define innovation in terms of it. We fully agree with Gajdon that the investigation into such mechanisms is necessary to understand innovation. Although there are ways to tell whether a behavior is novel without identifying specific mechanisms, knowledge of the mechanisms would provide a powerful way of deciding whether such behaviors are correctly classified and would be invaluable for cases that are difficult to classify.

Gajdon brings up the interesting case of individuals being more or less innovative in different environments: Keas in an aviary were more successful at completing a novel task than individuals in the wild. This is very important, and seems to complement observations of primates (e.g., Menzel 1966; van Schaik et al. 1999). Gardner's examples point to a developmental component to these environmental differences, indicating that the kinds of innovations produced by an individual depend on its accumulated experience. It is exactly to facilitate this kind of exciting work that we need to be clear about what we consider an innovation.

In answering Cachel's question as to whether some behaviors of social insects (such as ants) are innovations, it is useful to recall our second definition of innovation, which is posed in terms of repertoires. If the behavior "insects linking together to bridge a spatial gap," to use one of Cachel's examples - is part of the repertoire of the members of the species, then it is not to be considered an innovation. But if some insects modify their repertoire and thereby produce novel behaviors, innovation has occurred. There is no a priori reason to exclude insects or other small-brained taxa, though we feel that it is probable that the frequency and complexity of innovations will increase with increasing intelligence, as also suggested by the comparative studies reviewed earlier. This practical problem of recognizing innovations underscores how little we know about the cognitive dimensions of innovation, which future studies must address.

Reader asks whether "innovative propensities are adaptations rather than side-products or indicators of other 
phenomena, such as behavioral flexibility." Knowledge of the mechanisms involved is necessary to answer this question. If all of the abilities and dispositions that make one innovative have been selected for not because of the innovations they produce, but because of other roles they play (neophilia or general flexibility, say), then innovative propensities will be side-products. But if it can be shown that at least some elements of the mechanisms underlying innovative dispositions are the result of selection for the production of innovations, then the innovative disposition can be considered an adaptation.

\section{R3.2. Baldwin, learning, and innovation}

Hunt \& Gray claim that we presume that "the behavior of primates is generally learned and that of non-primates is generally 'innate'." In our article we offer no such claims. We do draw many of our examples from primates, but this is not presented as data confirming the claims (which we do not make) that primate behavior is "generally learned" and that the behavior of non-primates is "generally innate."

Hunt \& Gray also hold that we incorrectly characterize what we label "Baldwinized behaviors" as being not learned. Their claim is based on what they feel is a mistaken view of the Baldwin effect. We feel instead that their view of Baldwin is mistaken. (For a more positive take on our discussion, see McCall's treatment of the Baldwin effect and the myriad ways behavior can affect genetic evolution.) Baldwin (1896b) draws the distinction between natural and social heredity:

there is natural heredity by which variations are congenitally transmitted with original endowment, and there is "social heredity" by which functions socially acquired (i.e., imitatively, covering all the conscious acquisitions made through intercourse with other animals) are socially transmitted. The one is phylogenetic; the other ontogenetic. But these two lines of heredity influence are not separate nor uninfluential on each other. (Baldwin 1896b, p. 440)

By "natural heredity" Baldwin means innate and by "social heredity" he means social learning. His point is that social heredity can influence natural heredity, that is, that socially transmitted behaviors can become innate. In the target article, we made no claims about the degree to which this is realized in nature. Instead, our point was merely that if this process occurs, our operationization will not pick these behaviors out as innovations.

\section{R4. Conclusion}

Is our definition so restrictive that studying innovation is impossible? Several of the commentators (e.g., Giraldeau et al., Rendell et al., and Sargeant \& Mann) expressed skepticism about the usefulness of our definition. For example, Sargeant \& Mann hold that "most field studies will lack adequate data [to identify innovations in the field]." The first thing we would like to emphasize is that we need to distinguish between our definition of innovation and our method for recognizing innovations in the field. To point to our definition of innovation and claim that it makes the study of innovation impossible is like pointing to the definition of temperature - the mean kinetic energy of molecules or atoms - and claiming that because we (truly) cannot know the momentum of each molecule in some body, we could never know the temperature of that body. The reason that this is false is that we do not need to posses such knowledge in order to know the temperature. We can use indirect methods of measuring mean kinetic energy, such as the height of mercury in a tube or the degree of deflection of a bimetallic strip. Our definition of innovation is like that of temperature in three important ways: (1) At first blush it seems as if it is epistemologically intractable; that just as we cannot know the kinetic energy of the constituent molecules, we also cannot know that a behavior is both novel and learned, yet not simply a product of social learning or environmental induction. (2) Just as there are many (indirect and often simple) ways to measure temperature, so are there many ways to measure innovation. Our key is just one such method for discovering innovations and is meant to complement others. We welcome additional methods or modifications of our own method. (3) A criticism of the method of measuring temperature or innovativeness is not a criticism of the definition of temperature or innovation. Arguing that the deflection in a bimetallic strip is not a good way of measuring temperature is in no way an attack on the definition of temperature (in terms of mean kinetic energy). Many of the commentaries that were ostensibly critiquing our definition of innovation were, in fact, only critiquing our method for measuring innovations.

We appreciate the fact that our approach may make it harder to generate cases of innovation. But if the alternative is to produce conclusions of uncertain robustness, then we can only echo Charles Darwin's comment that "False facts are highly injurious to the progress of science, for they often endure long" (Darwin 1871, p. 385).

On the other hand, many of the disagreements the commentators had with the target article boil down to their having a different intuitive definition of innovation, much closer to our weak innovation, or even environmentally induced behavior. Some of this disagreement may be due to a different emphasis on function rather than mechanism or different taxonomic focus (here, birds vs. primates). The major difference is perhaps whether one is interested in ecology and macroevolution, as are most of our commentators, or in culture. Our interest in culture (see also Logan \& Pepper) has led us to emphasize stronger innovations that are not easily induced in individuals, and therefore must rely more on social learning to be transmitted and maintained in a population. In the end, whether the more liberal or more restrictive definition is used is, of course, up to each researcher, but the terminology used should reflect these differences. Most importantly, of course, future work should address the question of whether the more restrictive definition produces different conclusions than the more liberal one. Time will tell.

It is tempting to conclude from these difficulties, that we should give up on fieldwork and exclusively rely on experimental work in captivity. We agree that captive experiments can reveal the innovative tendencies of species and comparative experiments can be extremely insightful. However, there are two reasons not to limit our attention to captive experiments. First, conditions may affect the tendency to innovate, and we are only beginning to identify which these are; they may involve direct contextual but also 
References/Ramsey et al.: Animal innovation defined and operationalized

developmental variables. Because we should not expect all species to respond in the same way to these conditions, even captive experiments may not allow for direct comparisons between species. Second, captive work, no matter how ingenious, can give hints but will not permit decisive conclusions on the functional significance of any innovative tendencies in the wild. For instance, only fieldwork can help us decide the vexing question of whether innovative tendencies are byproducts of some other adaptive behavioral tendency or the direct product of natural selection. Fieldwork, which is bound to be more descriptive, given the serious limitations on experimentation in the field (the most serious one being the lack of control over the history of exposure of the animals), and experimental work in captivity are complementary; one cannot replace the other.

Deleporte argues convincingly that molecular phylogeography data would strengthen our approach. We agree and feel that a detailed knowledge of the population genetics and phylogeography of the populations under study would represent an important resource in the study of innovation, which could be used for a wide range of purposes, such as identifying predominant dispersal routes, deciding whether particular behaviors are innovations, identifying genetic influences on particular innovations, or studying the heritability of the individual trait of innovativeness. Indeed, one of us (van Schaik) is currently working on these issues for orangutans.

We are excited about other authors extending and strengthening the framework we have provided. Although we chiefly used foraging examples in the target article, several commentators pointed out that too little attention has been paid to innovations in other domains: use of space (Mettke-Hofmann), social signal variants (Locke), or sexual displays (Madden). And although our article focused on innovation in nonhuman animals, we are happy to see that Locke feels our framework will permit new perspectives on human behavior and evolution, such as the evolution of language.

The study of animal innovation is still a nascent field. Its central term - innovation - still has no fixed, agreed upon meaning. In order for this field to progress, and for research in the variety of disciplines studying innovation to complement each other, there needs to be a common framework that fixes the meaning of "innovation" and provides criteria for identifying innovations. We are hopeful that our target article will help create such a framework.

\section{References}

[The letters " $a$ " and " $r$ " before author's initials stand for target article and response references, respectively.]

Altmann, S. A. (1998) Foraging for survival: Yearling baboons in Africa. University of Chicago Press. [aGR]

Anderson, M. (1994) Sexual selection. Princeton University Press. [JRM]

Andrews, P. W., Gangestad, S. W. \& Matthews, D. (2002) Adaptationism: How to carry out an exaptationist program. Behavioral and Brain Sciences 25(4):489553. [PD]

Baker, M. C., Thompson, D. B., Sherman, G. L., Cunningham, M. A. \& Tomback, D. F. (1982) Allozyme frequencies in a linear series of song dialect populations. Evolution 38:449-51. [JRM]

Baldwin, J. M. (1896a) A new factor in evolution. American Naturalist 30:44151. [aGR, GRH, LM]

(1896b) Heredity and instinct. Science [New Series] 3:438-41. [rGR]

(1902) Development and evolution. Macmillian. [GRH]
Barbrook, A. C., Howe, C., Blake, J. N. \& Robinson, P. (1998) The phylogeny of The Canterbury Tales. Nature 394:839. [HV]

Bateson, P. (2003) The promise of behavioural biology. Animal Behaviour 65: 11-17. [CJL]

(2004) The active role of behaviour in evolution. Biology and Philosophy 19:28398. [GRH, LM]

Bateson, P. P. G. (1983) Genes, environment and the development of behaviour. In: Animal behaviour, vol. 3: Genes, development and learning, ed. T. R. Halliday \& P. J. B. Slater, pp. 52-81. Blackwell. [SMR]

(1988) The active role of behaviour in evolution. In: Evolutionary processes and metaphors, ed. M.-W. Ho \& S. W. Fox, pp. 191-207. Wiley. [LM]

Becker, G. S. (1976) The economic approach to human behavior. University of Chicago Press. [ELK]

Becker, J. A. (1994) "Sneak-shoes," "sworders" and "nose-beards": A case study of lexical innovation. First Language 14:195-211. [JLL]

Bergl, R. A. \& Vigilant, L. (2007) Genetic analysis reveals population structure and recent migration within the highly fragmented range of the Cross River gorilla (Gorilla gorilla gorilla). Molecular Ecology 16:501-16. [PD]

Bickerton, D. (1984) The language bioprogram hypothesis. Behavioral and Brain Sciences 7:173-88. [JLL]

Bischof, N. (1985) Das Rätsel Oedipus: Die biologischen Wurzeln des Urkonfliktes von Intimität und Autonomie. Piper. [GKG]

Bloom, K., D’Odorico, L. \& Beaumont, S. (1993) Adult preferences for syllabic vocalizations: Generalizations to parity and native language. Infant Behavior and Development 16:109-20. [JLL]

Bloom, K. \& Lo, E. (1990) Adult perceptions of vocalizing infants. Infant Behavior and Development 13209-19. [JLL]

Bodamer, M. D. \& Gardner, R. A. (2002) How cross-fostered chimpanzees initiate and maintain conversations. Journal of Comparative Psychology 116:12-26. [RAG]

Boesch, C. (1991) Teaching among wild chimpanzees. Animal Behaviour 41:53032. [RAG]

(1995) Innovation in wild chimpanzees (Pan troglodytes). International Journal of Primatology 16:1-16. [aGR]

(1996) The emergence of cultures among wild chimpanzees. In: Evolution of social behaviour patterns in primates and man, ed. W. G. Runciman, J. Maynard-Smith \& R. I. M. Dunbar. Oxford University Press. [aGR]

Boesch, C. \& Boesch, H. (1981) Sex differences in the use of natural hammers by wild chimpanzees: A preliminary report. Journal of Human Evolution 10:585-93. [aGR]

Boesch-Achermann, H. \& Boesch, C. (1993) Tool use in wild chimpanzees. Current Directions in Psychological Science 2:18-21. [RAG]

Box, H. O. (1984) Behavioral responses to environmental change: Some preliminary observations on common marmosets. Social Behavior and Human Affairs 49:81-89. [aGR]

Boyd, R. \& Richerson, P. J. (1985) Culture and evolutionary processes. University of Chicago Press. [BLS]

Burghardt, G. M. (2006) The genesis of animal play. MIT Press. [HV]

Burley, N. T \& Symanski, R. (1998) "A taste for the beautiful": Latent aesthetic mate preferences for white crests in two species of Australian grassfinches. American Naturalist 152:792-802. [JRM]

Byrne, R. W. (1995) The thinking ape: Evolutionary origins of intelligence. Oxford University Press. [aGR]

(2003) Novelty in deceit. In: Animal innovation, ed. S. M. Reader \& K. N. Laland, pp. 237-59. Oxford University Press. [AR, SMR]

Byrne, R. W. \& Corp, N. (2004) Neocortex size predicts deception rate in primates. Proceedings of the Royal Society of London B 271:1693-99. [aGR]

Byrne, R. W., Corp, N. \& Byrne, J. M. E. (2001a) Estimating the complexity of animal behaviour: How mountain gorillas eat thistles. Behaviour 138:525-57. [AR] (2001b) Manual dexterity in the gorilla: Bimanual and digit role differentiation in a natural task. Animal Cognition 4:347-61. [AR]

Cachel, S. (2006) Primate and human evolution. Cambridge University Press. [SC]

Caldwell, C. A. \& Whiten, A. (2002) Evolutionary perspectives on imitation: Is a comparative psychology of social learning possible? Animal Cognition5:193208. [aGR]

(2003) Scrounging facilitates social learning in common marmosets, Callithrix jacchus. Animal Behaviour 65:1085-92. [JRM]

Caro, T. M. \& Hauser, M. D. (1992) Is there teaching in nonhuman animals? The Quarterly Review of Biology 67:151-74. [GKG]

Cavalli-Sforza, L., Menozzi, P. \& Piazza, A. (1994) The history and geography of human genes. Princeton University Press. [PD]

Chalcraft, V. J. \& Gardner, R. A. (2005) Cross-fostered chimpanzees modulate signs of American Sign Language. Gesture 5:107-31. [RAG]

Chilvers, B. L. \& P. J. Corkeron (2001) Trawling and bottlenose dolphins' social structure. Proceedings of the Royal Society of London, Series B: Biological Sciences 268:1901-905. [CJL, BLS]

Coleman, K. \& Wilson, D. S. (1998) Shyness and boldness in Pumpkinseed sunfish: Individual differences are context specific. Animal Behaviour 56:927-36. [CM-H] 
References/Ramsey et al.: Animal innovation defined and operationalized

Custance, D. M., Whiten, A. \& Fredman, T. (1999) Social learning of an artificial fruit task in capuchin monkeys (Cebus apella). Journal of Comparative Psychology 113:13-23. [aGR]

Danchin, E., Giraldeau, L. A., Valone, T. J. \& Wagner, R. H. (2004) Public information: From nosy neighbors to cultural evolution. Science 305:487-91. [LM]

Darwin, C. R. (1871) The descent of man, and selection in relation to sex, vol. 2, 1st edition. John Murray. [rGR]

de Jong, G. \& Crozier, R. H. (2003) A flexible theory of evolution. Nature 424:1617. $[\mathrm{SMR}]$

Deaner, R.O., van Schaik, C. P. \& Johnson, V. (2006) Do some taxa have better domain-general cognition than others? A meta-analysis of nonhuman primate studies. Evolutionary Psychology 4:149-96. [aGR]

Dewar, G. (2004) Social and asocial cues about new food: Cue reliability influences intake in rats. Learning and Behavior 32:82-89. [SMR]

Dore, J. (1974) A pragmatic description of early language development. Journal of Psycholinguistics Research 4:343-50. [JLL]

Douadi, M., Gatti, S., Lévréro, F., Duhamel, G., Bermejo, M., Vallet, D., Ménard, N. \& Petit, E. J. (2007) Sex-biased dispersal in western lowland gorillas (Gorilla gorilla gorilla). Molecular Ecology 16:2247-59. [PD]

Drumm, P., Gardner, B. T. \& Gardner, R. A. (1986) Vocal and gestural responses of cross-fostered chimpanzees. American Journal of Psychology 99:1-29. [RAG]

Eckert, P. (1999) Linguistic variation as social practice. Blackwell. [JLL]

Eibl-Eibesfeldt, I. (1975) Ethology: The biology of behavior. Holt, Rinehart \& Winston. [aGR]

Elias, D. O., Hebets, E. A. \& Hoy, R. R. (2006) Female preference for complex/ novel signals in a spider. Behavioral Ecology 17:765-71. [JRM]

Emery, N. J. \& Clayton, N. S. (2004a) Comparing the complex cognition of birds and primates. In: Comparative vertebrate cognition: Are primates superior to non-primates?, ed. L. J. Rogers \& G. Kaplan. Kluwer Academic/Plenum. [GRH]

(2004b) The mentality of crows: Convergent evolution of intelligence in corvids and apes. Science 306:1903-907. [CJL]

Endler, J. A. \& Basolo, A. L. (1998) Sensory ecology, receiver biases and sexual selection. Trends in Ecology and Evolution 13:415-20. [JRM]

Erwin, D. H. \& Krakauer, D. C. (2004) Insights into innovation. Science 304:1117. [LM]

Fairbanks, L. A. (1993). Risk-taking by juvenile vervet monkeys. Behaviour 124:5772. $[\mathrm{GKG}]$

Fairbanks, L. A. \& McGuire, M. T. (1993) Maternal protectiveness and response to the unfamiliar in vervet monkeys. American Journal of Primatology 30:11929. [GKG]

Ferguson, C. A. (1964) Baby talk in six languages. American Anthropologist 66:10314. [JLL]

Fouts, R. S. \& Fouts, D. H. (1989) Loulis in conversation with the cross-fostered chimpanzees. In: Teaching sign language to chimpanzees, ed. R. A. Gardner, B. T. Gardner \& T. E. Van Cantfort, pp. 293-307. SUNY Press. [RAG]

Fouts, R. S., Fouts, D. H. \& Van Cantfort, T. E. (1989) The infant Loulis learns signs from cross-fostered chimpanzees. In: Teaching sign language to chimpanzees, ed. R. A. Gardner, B .T. Gardner \& T. E. Van Cantfort, pp. 280-92 SUNY Press. [RAG]

Fox, E. A., Sitompul, A. F. \& van Schaik, C. P. (1999) Intelligent tool use in wild Sumatran orangutans. In: The mentality of gorillas and orangutans, ed. S. Parker, L. Miles \& A. Mitchell. Cambridge University Press. [aGR]

Fox, E. A., van Schaik, C. P., Sitompul, A. \& Wright, D. N. (2004) Intra- and interpopulational differences in orangutan (Pongo pygmaeus) activity and diet: Implications for the invention of tool use. American Journal of Physical Anthropology 125:162-74. [aGR]

Fragaszy, D. \& Visalberghi, E. (2001) Recognizing a swan: Socially-biased learning Psychologia 44:82-98. [LM]

Fragaszy, D. M., Izar, P., Visalberghi, E., Ottoni, E. B. \& de Oliveria, M. G. (2004) Wild capuchin monkeys (Cebus libidinosus) use anvils and stone pounding tools. American Journal of Primatology 64:359-66. [aGR]

Fragaszy, D. M. \& Visalberghi, E. (1990) Social processes affecting the appearance of innovative behaviours in capuchin monkeys. Folia Primatologica 54:15565. $[\mathrm{SMR}]$

Freeman, S. \& Herron, J. C. (2004) Evolutionary analysis, 3rd edition. Pearson Education. [CJL]

Frith, C. B. \& Frith, D. W. (2004) Bowerbirds. Oxford University Press. [JRM]

Futuyma, D. (1998) Evolutionary biology 3rd edition. Sinauer. [LM]

Gajdon, G. K., Fijn, N. \& Huber, L. (2004) Testing social learning in a wild mountain parrot, the kea (Nestor notabilis). Learning and Behavior 32:6271. [GKG]

(2006) Limited spread of innovative behaviour in wild kea, Nestor notabilis. Animal Cognition 9:173-81. [GKG]

Galef, B. G. (1992) The question of animal culture. Human Nature 3:157-78. [HV]
Galef, Jr., B. G. (2003) “Traditional” foraging behaviors of brown and black rats (Rattus norvegicus and Rattus rattus). In: The biology of traditions: Models and evidence, ed. D. M. Fragaszy \& S. Perry. Cambridge University Press. [aGR]

Garcia, J. \& Koelling, R. A. (1966) Relation of cue to consequence in avoidance learning. Psychonomic Science 4:123-24. [aGR]

Gardner, B. T. \& Gardner, R. A. (1998) Development of phrases in the early utterances of children and cross-fostered chimpanzees. Human Evolution 13:161-88. [RAG]

Gardner, R. A. \& Gardner, B. T. (1989) A cross-fostering laboratory. In: Teaching sign language to chimpanzees, ed. R. A. Gardner, B. T. Gardner \& T. E. Van Cantfort, pp. 1-28. SUNY Press. [RAG]

(1998) Ethological study of early language. Human Evolution 13:189-207. [RAG]

Gardner, R. A., Gardner, B. T. \& Van Cantfort, T. E., eds. (1989) Teaching sign language to chimpanzees. SUNY Press. [RAG]

Gergely, G., Bekkering, H. \& Kiraly, I. (2002) Rational imitation in preverbal infants. Nature 415:755. [HV]

Gil, D., Cobb, J. L. S. \& Slater, P. J. B. (2001) Song characteristics are age dependent in the willow warbler, Phylloscopus trochilus. Animal Behaviour 62:689-94. [JRM]

Giraldeau, L. A. (1997) The ecology of information use. In: Behavioural Ecology, ed. J. R. Krebs \& N. B. Davies. Blackwell Scientific. [aGR]

Giraldeau, L., Soos, C. \& Beauchamp, G. (1994) A test of the producer-scrounger foraging game in captive flocks of spice finches, Lonchura punctulata. Behavioral Ecology and Sociobiology 34:251-56. [aGR]

Goldman, H. I. (2001) Parental reports of "mama" sounds in infants: An exploratory study. Journal of Child Language 28:497-506. [JLL]

Goodall, J. (1986) The chimpanzees of Gombe. Harvard University Press. [RAG]

Goossens, B., Chikhi, L., Jalil, M. F., Ancrenaz, M., Lackman-Ancrenaz, I., Mohamed, M., Andau, P. \& Bruford, M. W. (2006) Genetic signature of anthropogenic population collapse in orang-utans. Public Library of Science (PloS) Biology 4:0285-91. [PD]

Haemig, P. D. (1989) A comparative experimental study of exploratory behavior in Santa Cruz Island and mainland California scrubjays, Aphelocoma coerulescens. Bird Behavior 8:32-42. [GKG]

Hall, B. K. (2003) Baldwin and beyond: Organic selection and genetic assimilation. In: Evolution and learning: The Baldwin effect reconsidered, ed. B. Weber \& D. Depew. MIT Press. [GRH]

Handford, P. (1981) Vegetational correlates of variation in the song of Zonotrichia capensis. Behavioral Ecology and Sociobiology 8:203-206. [JRM]

Harris, M. P. (1970) Abnormal migration and hybridization of Larus argentatus and L. fuscus after interspecies fostering experiments. Ibis 112:488-98. [RAG]

Harvey, P. H. \& Purvis, A. (1991) Comparative methods for explaining adaptations. Nature 351:619-24. [CJL]

Hauser, M. D. (1988) Invention and social transmission: New data from wild vervet monkeys. In: Machiavellian intelligence: Social expertise and the evolution of intellect in monkeys, apes and humans, ed. R. W. Byrne \& A. Whiten, pp. 327-43. Oxford University Press. [aGR, SMR]

Healy, S. D. \& Rowe, C. (2007) A critique of comparative studies of brain size. Proceedings of the Royal Society of London, Series B: Biological Sciences 274:453-64. [rGR]

Henrich, J. (2001) Cultural transmission and the diffusion of innovations: Adoption dynamics indicate that biased cultural transmission is the predominate force in behavioral change. American Anthropologist 103:992-1013. [aGR]

Henrich, J. \& McElreath, R. (2003) The evolution of cultural evolution. Evolutionary Anthropology 12:123-35. [LM]

Herman, L. M. (2002) Exploring the cognitive world of the bottlenose dolphin. In: The cognitive animal, ed. M. Bekoff, C. Allen, \& G. M. Burghardt. MIT Press. [BLS]

Hinde, R. A. (1970) Animal behavior: A synthesis of ethology and comparative psychology, 2nd edition. McGraw-Hill. [aGR]

Hinde, R. A. \& Fisher, J. (1951) Further observations on the opening of milk bottles by birds. British Birds 44:393-96. [aGR]

(1972) Some comments on the re-publication of two papers on the opening of milk bottles by birds. In: Function and evolution of behavior, ed. P. H. Klopfer \& J. P. Hailman, pp. 377-78. Addison-Wesley. [HV]

Hohmann, G. \& Fruth, B. (2003) Intra- and inter-sexual aggression by bonobos in the context of mating. Behaviour 140:1389-1413. [aGR]

Holzhaider, J. C., Hunt, G. R., Campbell, V. M. \& Gray, R. D. (in press) Do wild New Caledonian crows (Corvus moneduloides) attend to the functional properties of their tools? Animal Cognition. [GRH]

Holzhaider, J. C., Hunt, G. R. \& Gray, R. D. (in preparation) The development of pandanus tool skills by juvenile New Caledonian crows. [GRH]

Hopkins, W. D., Taglialatela, J. P. \& Leavens, D. A. (2007) Chimpanzees differentially produce novel vocalizations to capture the attention of a human. Animal Behaviour 73:281-86. [JLL] 
References/Ramsey et al.: Animal innovation defined and operationalized

Hostetter, A. B., Cantero, M. \& Hopkins, W. D. (2001) Differential use of vocal and gestural communication by chimpanzees (Pan troglodytes) in response to the attentional status of a human (Homo sapiens). Journal of Comparative Psychology 115:337-43. [JLL]

Huffman, M. A. \& Hirata S. (2003) Biological and ecological foundations of primate behavioral traditions. In: The biology of traditions: Models and evidence, ed. D. M. Fragaszy \& S. Perry. Cambridge University Press. [aGR]

Hunt, G. R. (1996) Manufacture and use of hook-tools by New Caledonian crows Nature 379:249-51. [GRH]

(2005) Weird and wonderful artefacts. Trends in Ecology and Evolution 20:65556. [GRH]

Hunt, G. R., Abdelkrim, J., Anderson, M. G., Holzhaider, J. C., Marshall, A., Gemmell, N. \& Gray, R. D. (submitted) Innovative pandanustool folding by New Caledonian crows. Australian Journal of Zoology. [GRH]

Hunt, G. R. \& Gray, R. D. (2002) Species-wide manufacture of stick-type tools by New Caledonian crows. Emu 102:349-53. [GRH]

(2003) Diversification and cumulative evolution in tool manufacture by New Caledonian crows. Proceedings of the Royal Society of London B 270:86774. [GRH, CJL]

(2004) The crafting of hook tools by wild New Caledonian crows. Proceedings of the Royal Society of London B (Suppl.) 271:S88-S90. [GRH]

(2007) Parallel tool industries in New Caledonian crows. Biology Letters 3:17375. [GRH]

Hunt, G. R., Lambert, C. \& Gray, R. D. (2007) Cognitive requirements for tool use by New Caledonian crows (Corvus moneduloides). New Zealand Journal of Zoology 34:1-7. [GRH]

Hunt, G. R., Rutledge, R. B. \& Gray, R. D. (2006) The right tool for the job: What strategy do wild New Caledonian crows use? Animal Cognition 9:307-16. [GRH]

Imanishi, K. (1952) Man. Mainichi-Shinbunsha (in Japanese). [aGR]

Immelmann, K. \& Beer, C. (1989) A dictionary of ethology. Harvard University Press. [RAG]

Janik, V. M. \& Slater, P. J. B. (2000) The different roles of social learning in vocal communication. Animal Behaviour 60:1-11. [aGR]

Jantschke, F. (1972) Orang-utans in zoologischen garten. R. Piper. [aGR]

Jensvold, M. L. J. \& Gardner, R. A. (2000) Interactive use of sign language by cross-fostered chimpanzees. Journal of Comparative Psychology 114 335-46. [RAG]

Johnson, S. L. (2006) Do American robins acquire songs by both imitating and inventing? Wilson Journal of Ornithology 118:341-52. [JRM]

Johnson, V. E., Deaner, R. O. \& van Schaik, C. P. (2002) Bayesian analysis of multi-study rank data with application to primate intelligence ratings. Journal of the American Statistical Society 97:8-17. [aGR]

Kawamura, S. (1959) The process of sub-culture propagation among Japanese macaques. Primates 2:43-60. [aGR]

Kellogg, W. N. (1968) Communication and language in the home-raised chimpanzee. Science 162:423-27. [RAG]

Kendal, R. L., Coe, R. L. \& Laland, K. N. (2005) Age differences in neophilia, exploration and innovation in family groups of callitrichid monkeys. American Journal of Primatology 66:167-88. [RLK]

Kenward, B., Rutz, C., Weir, A. A. S., Chappell, J. \& Kacelnik, A. (2004) Morphology and sexual dimorphism of the New Caledonian crow Corvus moneduloides, with notes on its behaviour and ecology. Ibis 146:652-60. [GRH]

Kenward, B., Rutz, C., Weir, A. A. S. \& Kacelnik, A. (2006) Development of tool use in New Caledonian crows: Inherited action patterns and social influences. Animal Behavior 72:1329-43. [aGR]

Kenward, B., Weir, A. A. S., Rutz, C. \& Kacelnik, A. (2005) Behavioural ecology: Tool manufacture by naive juvenile crows. Nature 433:121 [aGR, GRH]

Khalil, E. L. (2000) Survival of the most foolish of fools: The limits of evolutionary selection theory. Journal of Bioeconomics 2:203-20. [ELK]

(2007) Natural selection and rational decision: Two concepts of optimization. A working paper. [ELK]

Kim, M. M., O’Connor, K. S., McLean, J., Robson, A. \& Chance, G. (1996) Do parents and professionals agree on the developmental status of high-risk infants? Pediatrics 97:676-81. [JLL]

Kirkpatrick, M. \& Dugatkin, L. A. (1994) Sexual selection and the evolutionary effects of copying mate choice. Behavioral Ecology and Sociobiology 34:44349. [JRM]

Kothbauer-Hellman, R. (1990) On the origin of a tradition: Milk bottle opening by titmice. Zoologischer Anzeiger 225:353-61. [SMR]

Kreps, D. M. (1990) A course in microeconomic theory. Princeton University Press. $\quad[\mathrm{ELK}]$

Kroodsma, D. (2004) The diversity and plasticity of birdsong. In: Nature's music: The science of birdsong, ed. P. Marler \& H. Slabberkoorn, pp. 108-31. Elsevier/Academic Press. [JRM]
Krützen, M., Mann, J., Heithaus, M. R., Connor, R. C., Bejder, L. \& Sherwin, W. B. (2005) Cultural transmission of tool use in bottlenose dolphins. Proceedings of the National Academy of Sciences USA 102:8939-43. [BLS]

Kummer, H. (1971) Primate societies: Group techniques of ecological adaptation. AHM Publishing. [aGR]

Kummer, H. \& Goodall, J. (1985) Conditions of innovative behaviour in primates. Philosophical Transactions of the Royal Society of London B 308:203-14. [L-AG, GKG, RKL, aGR]

Labov, W. (2001) Principles of linguistic change, vol. 2: Social factors. Blackwell. [JLL]

Laland, K. N. (1994) Sexual selection with a culturally transmitted mating preference. Theoretical Population Biology 45:1-15. [JRM]

Laland, K. N. \& Hoppitt, W. (2003) Do animals have culture? Evolutionary Anthropology 12:150-59. [CJL]

Laland, K. N. \& Janik, V. (2006) The animal cultures debate. Trends in Ecology and Evolution 21(10):542-47. [RLK, CJL, aGR, SMR, BLS]

Laland, K. N. \& Kendal, J. (2003) What the models say about social learning. In: Biology of traditions, ed. D. Fragaszy \& S. Perry. Cambridge University Press. [LM]

Laland, K. N., Kendal, J. R. \& Kendal, R. L. (in press) Animal culture: Problems and solutions. In: The question of animal culture, ed. K. N. Laland \& B. G. Galef Jr. Harvard University Press. [RLK]

Laland, K. N. \& Reader, S. M. (1999) Foraging innovation in the guppy. Animal Behaviour 57:331-40. [RLK]

Leatherwood, S. (1975) Some observations of feeding behavior of bottle-nosed dolphins (Tursiops truncatus) in the Northern Gulf of Mexico and (Tursiops cf T. gilli) off Southern California, Baja California, and Nayarit, Mexico. Marine Fisheries Review 37(9):10-16. [CJL]

Leavens, D. A., Hostetter, A. B., Wesley, M. J., \& Hopkins, W. D. (2004) Tactical use of unimodal and bimodal communication by chimpanzees, Pan troglodytes. Animal Behaviour 67:467-76. [JLL]

Lee, P. (1991) Adaptations to environmental change: An evolutionary perspective. In: Primate responses to environmental change, ed. H. O. Box, pp. 39-56. Chapman \& Hall. [SMR]

Lefebvre, L. (2000) Feeding innovations and their cultural transmission in bird populations. In: The evolution of cognition, ed. C. M. Heyes \& L. Huber. MIT Press. [aGR]

Lefebvre, L. \& Bolhuis, J. (2003) Positive and negative correlates of feeding innovations in birds: Evidence for limited modularity. In: Animal innovation, ed. S. M. Reader \& K. N. Laland. Oxford University Press. [GKG, aGR]

Lefebvre, L., Gaxiola, A., Dawson, S., Timmermans, S., Rosza, L. \& Kabai, P. (1998) Feeding innovations and forebrain size in Australian birds. Behaviour 135:1077-97. [GKG, rGR]

Lefebvre, L., Nicolakakis, N. \& Boire, D. (2002) Tools and brains in birds. Behaviour 139:939-73. [GRH]

Lefebvre, L., Reader, S. M. \& Sol, D. (2004) Brains, innovations and evolution in birds and primates. Brain, Behavior and Evolution 63(4):233-46. [CM-H, RLK, JLL, arGR]

Lefebvre, L., Reader, S. M. \& Webster, S. J. (2001) Novel food use by Gray Kingbirds and Red-necked Pigeons in Barbados. Bulletin of the British Ornithologists' Club 121:247-49. [SMR]

Lefebvre, L., Whittle, P., Lascaris, E. \& Finkelstein, A. (1997) Feeding innovations and forebrain size in birds. Animal Behaviour 53:549-60. [arGR, SMR]

Leopold, W. F. (1949) Original invention in infant language. Symposium 3:6675. [JLL]

Levins, R. (1968) Evolution in changing environments. Princeton University Press. [aGR]

Lloyd Morgan, C. (1896) On modification and variation. Science 4:733-40. [LM

Locke, J. L. (2004) Trickle up phonetics: A vocal role for the infant. Behavioral and Brain Sciences 27:516. [JLL]

(in press) Evolutionary developmental linguistics: Naturalization of the faculty of language. Language Sciences. [JLL]

Locke, J. L. \& Bogin, B. (2006) Language and life history: A new perspective on the evolution and development of linguistic communication. Behavioral and Brain Sciences 29(3):259-325. [JLL]

Lonsdorf, E. V., Eberly, L. E. \& Pusey, A. E. (2004) Sex differences in learning in chimpanzees. Nature 428:715-16. [BLS]

Madden, J. R. \& Balmford, A. P. (2004) Spotted bowerbirds Chlamydera maculata do not prefer rare or costly bower decorations. Behavioral Ecology and Sociobiology 55:589-95. [JRM]

Madden, J. R., Lowe, T. J., Fuller, H. V., Dasmahapatra, K. K. \& Coe, R. L. (2004) Local traditions of bower decoration by spotted bowerbirds in a single population. Animal Behaviour 68:759-65. [JRM]

Mann, J. \& Kemps, C. (2003) The effects of provisioning on maternal care in wild bottlenose dolphins, Shark Bay, Australia. In: Marine mammals: Fisheries, tourism and management issues, ed. N. Gales, M. Hindell \& R. Kirkwood. CSIRO Publishing. [BLS] 
References/Ramsey et al.: Animal innovation defined and operationalized

Mann, J. \& Sargeant, B. (2003) Like mother, like calf: The ontogeny of foraging traditions in wild Indian Ocean bottlenose dolphins (Tursiops sp.). In: The biology of traditions: Models and evidence, ed. D. M. Fragaszy \& S. Perry. Cambridge University Press. [BLS]

Marino, L., Connor, R. C., Fordyce, R. E., Herman, L. M., Hof, P. R., Lefebvre, L. Lusseau, D., McCowan, B., Nimchinsky, E. A., Pack, A.A., Rendell, L., Reidenberg, J. S., Reiss, D., Uhen, M. D., Van der Gucht, E., \& Whitehead, H (2007) Cetaceans have complex brains for complex cognition. PLoS Biology 5(5):e139. [CJL]

Marler, P. (1991) The instinct to learn. In: The epigenesis of mind: Essays on biology and cognition, ed. S. Carey \& R. Gelman. Erlbaum. [JLL]

Marler, P. \& Slabberkoorn, H. (2004) Nature's music: The science of birdsong. Elsevier/Academic Press. [JRM]

Marler, P. \& Tamura, M. (1964) Culturally transmitted patterns of vocal behaviour in sparrows. Science 146:1483-86. [JRM]

Martin, L. B., II. \& Fitzgerald, L. (2005) A taste for novelty in invading house sparrows, Passer domesticus. Behavioral Ecology 16:702-707. [CM-H]

Matsuzawa, T. (1994) Field experiments on use of stone tools by chimpanzees in the wild. In: Chimpanzee cultures, ed. R. W. Wrangham, W. C. McGrew, F. B. M. de Waal \& P. G. Heltne. Harvard University Press. [aGR]

McCune, L., Vihman, M. M., Roug-Hellichius, L., Delery, D. B. \& Gogate, L. (1996) Grunt communication in human infants (Homo sapiens). Journal of Comparative Psychology 110:27-37. [JLL]

McDougall, P. T., Réale, D., Sol, D. \& Reader, S. M. (2006) Wildlife conservation and animal temperament: Causes and consequences of evolutionary change for captive, reintroduced, and wild populations. Animal Conservation 9:39-48. [SMR]

McGrew, W. C. (1998) Culture in nonhuman primates? Annual Review of Anthropology 27:310-28. [aGR]

(2004) Invention and innovation. Nature 427:679. [aGR]

McGrew, W. C. \& Tutin, C. E. G. (1978) Evidence for a social custom in wild chimpanzees? Man 13:234-51. [aGR]

Menzel, E. W., Jr. (1966) Reponsiveness to objects in free-ranging Japanese monkeys. Behaviour 26:130-50. [rGR]

Mettke-Hofmann, C. (2007) Object exploration of garden and Sardinian warblers peaks in spring. Ethology 113:174-82. [CM-H]

Mettke-Hofmann, C., Ebert, C., Schmidt, T., Steiger, S. \& Stieb, S. (2005a) Personality traits in resident and migratory warbler species. Behaviour 142:135775. [GKG, CM-H]

Mettke-Hofmann, C., Manthej, S., Schlicht, E., Schneider, J. \& Werner, F. (under review) Spatial neophilia and neophobia in resident and migratory warblers (Sylvia). [CM-H]

Mettke-Hofmann, C., Wink, M., Winkler, H. \& Leisler, B. (2005b) Exploration of environmental changes relates to lifestyle. Behavioral Ecology 16:247-54. [CM-H]

Mettke-Hofmann, C., Winkler, H. \& Leisler B. (2002) The significance of ecological factors for exploration and neophobia in parrots. Ethology 108:249-72. [GKG, aGR]

Miller, G. (2000) The mating mind: How sexual choice shaped the evolution of human nature. William Heinemann. [JRM]

Morand-Ferron, J., Lefebvre, L., Reader, S. M., Sol, D. \& Elvin, S. (2004) Dunking behaviour in Carib grackles. Animal Behaviour 68:1267-74. [RLK, arGR, SMR]

Nicolakakis, N. \& Lefebvre, L. (2000) Forebrain size and innovation rate in European birds: Feeding, nesting and confounding variables. Behaviour 137:1415-27. [RLK, aGR]

Ninio, A., Snow, C., Pan, B. A. \& Rollins, P. R. (1994) Classifying communicative acts in children's interactions. Journal of Communication Disorders 27:15887. [JLL]

Odling-Smee, F. J., Laland, K. N. \& Feldman, M. W. (2003) Niche construction: The neglected process in evolution. Princeton University Press. [LM, aGR]

Oller, D. K. (2000) The emergence of the speech capacity. Erlbaum. [JLL] (2004) Underpinnings for a theory of communicative evolution. In: The evolution of communication systems: A comparative approach, ed. D. K. Oller \& U. Griebel. MIT Press. [JLL]

O’Neill, D. K. \& Happé, G. E. (2000) Noticing and commenting on what's new: Differences and similarities among 22-month-old typically developing children, children with Down syndrome and children with autism. Developmental Science 3:457-78. [JLL]

Parker, S. Z., Kerr, M., Markowitz, H. \& Gould, J. (1999) A survey of tool use in zoo gorillas. In: The mentalities of gorillas and orangutans: Comparative perspectives, ed. S. T. Parker, R. W. Mitchell \& H. L. Miles. Cambridge University Press. [aGR]

Pawlby, S. J. (1977) Imitative interaction. In: Studies in mother-infant interaction, ed. H. R. Schaffer. Academic Press. [JLL]

Perry, S. (2003) Conclusions and research agendas. In: The biology of traditions, ed. \begin{tabular}{l} 
D. M. Fragaszy \& S. Perry, pp. 426-40. Cambridge University Press. [CJL] \\
\hline
\end{tabular}
Perry, S. \& Manson, J. H. (2003) Traditions in monkeys. Evolutionary Anthropology 12:71-81. [aGR]

Perry, S., Panger, M., Rose, L. M., Baker, M., Gros-Louis, J., Jack, K., MacKinnon, K. C., Manson, J., Fedigan, L. \& Pyle, K. (2003) Traditions in wild white-faced capuchin monkeys. In: The biology of traditions: Models and evidence, ed. D. M. Fragaszy \& S. Perry. Cambridge University Press. [aGR]

Pfeffer, K., Fritz, J. \& Kotrschal, K. (2002) Hormonal correlates of being an innovative greylag goose, Anser anser. Animal Behaviour 63:687-95. [SMR]

Podos, J., Huber, J. K. \& Taft, B. (2004) Bird song: The interface of evolution and mechanism. Annual Review of Ecology, Evolution and Systematics 35:5587. [HV]

Pogue, M. G. \& Mickevich, M. F. (1990) Character definitions and character state delineation: The bête noire of phylogenetic inference. Cladistics 6:319-61. [PD]

Previde, E. P. \& Poli, M. D. (1996) Social learning in the Golden Hamster. Journal of Comparative Psychology 110:203-08. [RAG]

Reader, S. M. (2003) Innovation and social learning: Individual variation and brain evolution. Animal Biology 53:147-58. [CM-H, aGR]

(2004) Distinguishing social and asocial learning using diffusion dynamics. Learning \& Behavior 32:90-104. [aGR, SMR]

(2006) Brain size in primates as a function of behavioral innovation. In: Evolution of nervous systems, vol. V: The evolution of primate nervous systems, ed. T. M. Preuss \& J. H. Kaas, pp. 261-66. Academic Press. [RLK]

Reader, S. M. \& Laland, K. N. (2001) Primate innovation: Sex, age and social rank differences. International Journal of Primatology 22:787-805. [RLK, JLL, aGR, SMR]

(2002) Social intelligence, innovation and enhanced brain size in primates. Proceedings of the National Academy of Sciences USA 99:4436-41. [GRH, RLK, arGR, SMR, BLS]

(2003a) Animal innovation: An introduction. In: Animal innovation, ed. S. M. Reader \& K. N. Laland, pp. 3-35. Oxford University Press. [RLK, AR, $\operatorname{arGR}, \mathrm{SMR}, \mathrm{BLS}]$

Reader, S. M. \& Laland, K. N., eds. (2003b) Animal innovation. Oxford University Press. [RLK, LM, aGR, BLS]

Reader, S. M. \& MacDonald, K. (2003) Environmental variability and primate behavioural flexibility. In: Animal innovation, ed. S. M. Reader \& K. N. Laland, pp. 83-116. Oxford University Press. [rGR]

Reader, S. M., Morand-Ferron, J., Côté, I. \& Lefebvre, L. (2002a) Unusual feeding behaviors in five species of Barbadian birds. El Pitirre 15:117-23. [SMR]

Reader, S. M., Nover, D. \& Lefebvre, L. (2002b) Locale-specific sugar packet opening by Lesser Antillean bullfinches in Barbados. Journal of Field Ornithology 73:82-85. [SMR]

Réale, D., Reader, S. M., Sol, D., McDougall, P. T. \& Dingemanse, N. J. (2007) Integrating animal temperament within ecology and evolution. Biological Reviews 82:291-318. [SMR]

Rendell, L. \& Whitehead, H. (2001) Culture in whales and dolphins. Behavioral and Brain Sciences 24(2):309-82. [aGR, BLS]

Rodd, F. H., Hughes, K. A., Grether, G. F. \& Baril, C. T. (2002) A possible nonsexual origin of mate preference: Are male guppies mimicking fruit? Proceedings of the Royal Society of London, Series B: Biological Sciences 269:47581. [JRM]

Rogers, A. R. (1988) Does biology constrain culture? American Anthropologist 90:819-31. [LM]

Rogers, E. M. (1983) Diffusion of innovations. The Free Press. [aGR]

Rowley, I. \& Chapman, G. (1986) Cross-fostering, imprinting and learning in two sympatric species of cockatoos. Behaviour 96:1-16. [RAG]

Rumbaugh, D. M. \& Washburn, D. A. (2003) Intelligence of apes and other rational beings. Yale University Press. [aGR]

Rumbaugh, D. M., Washburn, D. A. \& Hillix, W. A. (1996) Respondants, operants, and emergents: Toward an integrated perspective on behavior. In: Learning as self-organizing process, ed. K. Pribram \& J. King. Erlbaum. [aGR]

Russell, J., Braccini, S., Buehler, N., Kachin, M., Schapiro, S. J. \& Hopkins, W. D. (2005) Chimpanzees (Pan troglodytes) intentional communication is not contingent upon food. Animal Cognition 8:263-72. [JLL]

Russon, A. E. (2003) Innovation and creativity in forest-living rehabilitant orangutans. In: Animal innovation, ed. S. M. Reader \& K. N. Laland, pp. 279-306. Oxford University Press. [AR]

Russon, A. E. \& Galdikas. B. M. (1995) Constraints on great apes' imitation: Model and action selectivity in rehabilitant orangutan (Pongo pygmaeus) imitation. Journal of Comparative Psychology 109:5-17. [aGR]

Russon, A. E., Handayani, P., Kuncoro, P. \& Ferisa, A. (2006) Orangutan leafcarrying for nest building: Unravelling cultural processes. Animal Cognition (online: DOI 10.1007/s10071-006-0058-z). [AR]

Sargeant, B. L., Mann, J., Berggren, P. \& Krützen, M. (2005) Specialization and development of beach hunting, a rare foraging behavior, by wild bottlenose dolphins (Tursiops sp.). Canadian Journal of Zoology 83:1400-10. [BLS] 
Sargeant, B. L., Wirsing, A. J., Heithaus, M. R. \& Mann, J. (2007) Can environmental heterogeneity explain individual foraging variation in wild bottlenose dolphins (Tursiops sp.)? Behavioral Ecology and Sociobiology 61:679-88. [BLS]

Schlichting, C. D. \& Pigliucci, M. (1998) Phenotypic evolution: A reaction norm perspective. Sinauer. [SMR]

Scott, J. P. (1958) Animal behavior. The University of Chicago Press. [RAG]

Shaw, H. L. (2000) Gaze direction in conversational interactions of chimpanzees. Unpublished doctoral dissertation, University of Nevada, Reno. [RAG]

Sherry, D. F. \& Galef, B. G., Jr. (1984) Cultural transmission without imitation: Milk bottle opening by birds. Animal Behaviour 32(3):937-38. [SMR, HV]

(1990) Social learning without imitation: More about milk bottle opening by birds. Animal Behaviour 40(5):987-89. [SMR]

Sih, A., Bell, A. M., Johnson, J. C. \& Ziemba, R. E. (2004) Behavioral syndromes: An integrative overview. Quarterly Review of Biology 79:241-77. [SMR]

Simonton, D. K. (2003) Human creativity: Two Darwinian analyses. In: Animal innovation, ed. S. M. Reader \& K. N. Laland. Oxford University Press. [aGR]

Skinner, B. F. (1935) The generic nature of the concepts of stimulus and response. Journal of General Psychology 12:40-65. [aGR]

Slabbekoorn, H., Jesse, A. \& Bell, D. A. (2003) Microgeographic song variation in island populations of the white-crowned sparrow (Zonotrichia leucophrys nutalli): Innovation through recombination. Behaviour 140:947-63. [JRM]

Slater, P. J. B. \& Lachlan, R. F. (2003) Is innovation in bird song adaptive? In: Animal innovation, ed. S. M. Reader \& K. N. Laland, pp. 117-36. Oxford University Press. [aGR, HV]

Smith, B. L. \& Oller, D. K. (1981) A comparative study of pre-meaningful vocalizations produced by normally developing and Down's syndrome infants. Journal of Speech and Hearing Disorders 46:46-51. [JLL]

Smith, V. A., King, A. P. \& West, M. J. (2002) The context of social learning: Association patterns in a captive flock of brown-headed cowbirds. Animal Behaviour 63:23-35. [CJL]

Sol, D. (2003) Behavioral innovation: A neglected issue in the ecological and evolutionary literature? In: Animal innovation, ed. S. M. Reader \& K. N. Laland, pp. 63-82. Oxford University Press. [aGR, SMR]

Sol, D., Duncan R. P., Blackburn, T. M., Cassey P. \& Lefebvre, L. (2005a) Big brains, enhanced cognition, and response of birds to novel environments. Proceedings of the National Academy of Sciences 102:5460-65. [arGR]

Sol, D., Lefebvre, L. \& Domingo Rodriguez-Teijeiro, J. (2005b) Brain size, innovative propensity and migratory behaviour in temperate Palaearctic birds. Proceedings of the Royal Society of London B 272:1433-41. [CM-H]

Sperber, D. (2001) An objection to the memetic approach to culture. In: Darwinizing culture: The status of memetics as a science, ed. R. Aunger, pp. 163-73. Oxford University Press. [HV]

Stamps, J. (2003) Behavioral processes affecting development: Tinbergen's fourth question comes of age. Animal Behaviour 66:1-13. [RAG]

Sterelny, K. (2004) A review of Evolution and learning: The Baldwin effect reconsidered, ed. B. Webber \& D. Depew. Evolution and Development 6:295300. [GRH]

Swaddle, J. P., Cathey, M. G., Correll, M. \& Hodkinson, B. P. (2005) Socially transmitted mate preferences in a monogamous bird: A non-genetic mechan ism of sexual selection. Proceedings of the Royal Society of London B: Biological Sciences 272:1053-58. [JRM]

Taylor, A. H., Hunt, G. R., Holzhaider, J. C. \& Gray, R. D. (2007) Spontaneous metatool use by New Caledonian crows. Current Biology 17:1504-1507. [GRH]

Tebbich, S., Taborsky, M., Fessl, B. \& Blomqvist, D. (2001) Do woodpecker finches acquire tool-use by social learning? Proceedings of the Royal Society of London B 268: 2189-93. [aGR, GRH]

Templeton, A. R. (1998) Nested clade analyses of phylogeographic data: Testing hypotheses about gene flow and population history. Molecular Ecology 7:38197. $[\mathrm{PD}]$

ten Cate, C. \& Rowe, C. (2007) Biases in signal evolution: Learning makes a difference. Trends in Ecology and Evolution 22:380-87. [JRM]

Terrace, H. S., Pettito, L., Sanders, R. J. \& Bever, T. G. (1979) Can an ape create a sentence? Science 206:891-902. [RAG]

Thornton, A. \& McAuliffe, K. (2006) Teaching in wild meerkats. Science 313:22729. [BLS]

Thorpe, W. H. (1943) A type of insight learning in birds. British Birds 37:29-31. [L-AG]

(1956) Records of the development of unusual and original feeding methods by wild passerine birds. British Birds 49:389-95. [L-AG]

Timmermans, S., Lefebvre, L., Boire, D. \& Basu, P. (2000) Relative size of the hyperstriatum ventrale is the best predictor of feeding innovation rate in birds. Brain, Behavior and Evolution 56: 196-203. [GKG, rGR]

Tomasello, M., George, B., Kruger, A., Farrar, J. \& Evans, E. (1985) The development of gestural communication in young chimpanzees. Journal of Human Evolution 14:175-86. [JLL]
Tomasello, M., Gust, D. \& Frost, G. T. (1989) The development of gestural communication in young chimpanzees: A follow up. Primates 30:35-50. [JLL]

Trivers, R. L. (1974) Parent-offspring conflict. American Zoologist 14:249-64. [JLL]

Tullock, G. (1971) The coal tit as a careful shopper. American Naturalist 105:7780. [ELK $]$

Uy, J. A. C. \& Borgia, G. (2000) Sexual selection drives rapid divergence in bowerbird display traits. Evolution 54:273-78. [JRM]

Uy, J. A. C., Patricelli, G. L. \& Borgia, G. (2001) Complex mate searching in the satin bowerbird Ptilonorhynchus violaceus. American Naturalist 158:53042. [JRM]

van Schaik, C. P. (2003) Local traditions in orangutans and chimpanzees: Social learning and social tolerance. In: The biology of traditions: Models and evidence, ed. D. M. Fragaszy \& S. Perry. Cambridge University Press. [aGR]

(in press) Geographic variation in the behavior of wild great apes: Is it really cultural? In: The question of culture, ed. B. G. Galef \& K. Laland. [aGR]

van Schaik, C. P., Ancrenaz, M., Borgen, G., Galdikas, B., Knott, C. D., Singleton, I., Suzuki, A., Utami, S. S. \& Merrill, M. Y. (2003a) Orangutan cultures and the evolution of material culture. Science 299:102-105. [aGR]

van Schaik, C. P., Deaner, R. O. \& Merrill, M. Y. (1999) The conditions for tool use in primates: Implications for the evolution of material culture. Journal of Human Evolution 36:719-41. [rGR]

van Schaik, C. P., Fox, E. A. \& Fechtman, L. T. (2003b) Individual variation in the rate of use of tree-hole tools among wild orang-utans: Implications for hominin evolution. Journal of Human Evolution 44:11-23. [aGR]

van Schaik, C. P., Fox, E. A. \& Sitompul, A. F. (1996) Manufacture and use of tools in wild Sumatran orangutans. Naturwissenschaften 83:186-88. [aGR]

van Schaik, C. P. \& Pradhan, G. R. (2003) A model for tool-use traditions in primates: Implications for the coevolution of culture and cognition. Journal of Human Evolution 44:645-64. [aGR]

van Schaik, C. P., van Noordwijk, M. A. \& Wich, S. A. (2006) Innovation in wild Bornean orangutans (Pongo pygmaeus wurmbii). Behaviour 143(7):839-76. [CM-H, AR, arGR]

Vellenga, R. (1986) Bower-building behaviour of immature satin bowerbirds Ptilonorhynchus violaceus. Australian Bird Watcher 11:166-67. [JRM]

Veneziano, E. (1988) Vocal-verbal interaction and the construction of early lexical knowledge. In: The emergent lexicon: The child's development of a linguistic vocabulary, ed. M. D. Smith \& J. L. Locke. Academic Press. [JLL]

Visalberghi, E. \& Fragaszy, D. M. (1990) Food-washing behaviour in tufted capuchin monkeys, Cebus apella, and crabeating macaques, Macaca fascicularis. Animal Behaviour 40:829-36. [SMR]

Visalberghi, E. \& Limongelli, L. (1994) Lack of comprehension of cause-effect relations in tool-using capuchin monkeys (Cebus apella). Journal of Comparative Psychology 108:15-22. [aGR]

Waddington, C. H. (1942) The canalization of development and the inheritance of acquired characters. Nature 150:563-65. [GRH]

(1953) Genetic assimilation of an acquired character. Evolution 7:118-26. [GRH]

(1957) The strategy of the genes: A discussion of some aspects of theoretical biology. George Allen \& Unwin. [LM]

Waples, R. S. \& Gaggiotti, O. E. (2006) What is a population? An empirical evaluation of some genetic methods for identifying the number of gene pools and their degree of connectivity. Molecular Ecology 15:1419-39. [PD]

Watanabe, K. (1994) Precultural behavior of Japanese macaques: Longitudinal studies of the Koshima troops. In: The ethological roots of culture, ed. R.A. Gardner, B.T. Gardner, A.B. Chiarelli \& F.X. Plooij, pp. 81-94. Kluwer. [RAG]

Weber, B. \& Depew, D., eds. (2003) Evolution and learning: The Baldwin effect reconsidered. MIT Press. [GRH]

Webster, S. J. \& Lefebvre, L. (2001) Problem solving and neophobia in a columbiform-passeriform assemblage in Barbados. Animal Behaviour 62:23-32. [CM-H]

Weir, A. S., Chappell, J. \& Kacelnik, A. (2002) Shaping of hooks in New Caledonian crows. Science 297:981. [GRH]

Weir, A. S. \& Kacelnik, A. (2006) A New Caledonian crow (Corvus moneduloides) creatively re-designs tools by bending or unbending aluminium strips. Animal Cognition 9:317-34. [GRH]

West-Eberhard, M. J. (2003) Developmental plasticity and evolution. Oxford University Press. [GRH, JLL, aGR]

Whitehead, H. (2007) Learning, climate and evolution of cultural capacity. Journal of Theoretical Biology 245:341-50. [BLS]

Whitehead, H., Rendell, L., Osborne, R. W. \& Wursig, B. (2004) Culture and conservation of non-humans with reference to whales and dolphins: Review and new directions. Biological Conservation 120(3):427-37. [CJL]

Whiten, A., Goodall, J., McGrew, W. C., Nishida, T., Reynolds, V., Sugiyama, Y., Tutin, C. E. G., Wrangham, R. W. \& Boesch, C. (1999) Cultures in chimpanzees. Nature 399:682-85. [aGR, GRH] 
References/Ramsey et al.: Animal innovation defined and operationalized

(2001) Charting cultural variation in chimpanzees. Behaviour 138:1481-1516. [aGR]

Whiten, A., Horner, V. \& de Waal, F. B. M. (2005) Conformity to cultural norms of tool use in chimpanzees. Nature 437:737-40. [CJL, aGR]

Whiten, A. \& van Schaik, C. P. (2007) The evolution of animal "cultures" and social intelligence. Philosophical Transactions of the Royal Society B 362:603-20. [aGR]

Whittaker, R. H. (1972) Evolution and measurement of species diversity. Taxon 21:213-51. [aGR]
Wyles, J. S., Kundel, J. G. \& Wilson, A. C. (1983) Birds, behaviour, and anatomical evolution. Proceedings of the National Academy of Sciences of the USA 80:4394-97. [aGR, SMR]

Yoerg, S. I. (2001) Clever as a fox: Animal intelligence and what it can teach us about ourselves. Bloomsbury. [aGR]

Yurk, H., Barrett-Lennard, L., Ford, J. K. B. \& Matkin, C. O. (2002) Cultural transmission within maternal lineages: Vocal clans in

resident killer whales in southern Alaska. Animal Behaviour 63:1103-19. [CJL] 